\title{
Kosterlitz-Thouless Transition Line for the Two Dimensional Coulomb Gas
}

\author{
Pierluigi Falco
}

September 9, 2018

\begin{abstract}
With a rigorous renormalization group approach, we study the pressure of the two dimensional Coulomb Gas along a small piece of the KosterlitzThouless transition line, i.e. the boundary of the dipole region in the activitytemperature phase-space.
\end{abstract}

\section{Contents}

1 Introduction

2 Definitions and Results 4

3 Strategy of the Proof 6

4 Renormalization Group Map 11

4.1 First RG step . . . . . . . . . . . . . . . . . . 12

$4.2 \quad j$-th RG Step . . . . . . . . . . . . . . . . . . . 13

5 Estimates $\quad 19$

5.1 Norms and Regulators . . . . . . . . . . . . . . . . 19

5.2 Power Counting Analysis . . . . . . . . . . . . . . 23

6 Smoothness of RG Map 24

6.1 Proof of Lemma 3.3 and $3.2 \ldots \ldots \ldots \ldots$. . . . . . . . . . . . . . . . . . . . . . . . . . . .

6.2 Proof of Theorem $3.4 \ldots \ldots \ldots \ldots$. . . . . . . . . . . . . . . . . . . . . . . . . . . . .

6.3 Preparation Bounds . . . . . . . . . . . . . . 29

6.4 Proof of Theorem 3.5 . . . . . . . . . . . . . . . . . . 33

7 Stable Manifold Theorem 37 
A Functional Integral Formula 41

A.1 Siegert-Kac transformation . . . . . . . . . . . . . . . . . 41

A.2 Multiscale decomposition. . . . . . . . . . . . . . . . . 43

A.3 Explicit Computations . . . . . . . . . . . . . . 46

B Charged Components 49

C Lowest Orders Computation 49

C.1 First Order . . . . . . . . . . . . . . . . . . . 49

C.2 Second Order . . . . . . . . . . . . . . . . . . . . 49

C.3 Estimates for $W_{j} \ldots \ldots \ldots \ldots \ldots \ldots$

D Proof of Lemma 5.2, 52

E Proof of Theorems $5.5,5.6$ and 5.7

E.1 Proof of Theorem $5.6 \ldots \ldots \ldots \ldots \ldots \ldots$

E.2 Proof of Theorem $5.5 \ldots \ldots \ldots \ldots \ldots \ldots$

E.3 Proof of Theorem $5.7 \ldots \ldots \ldots \ldots \ldots$

\section{Introduction}

Occasionally in Statistical Mechanics the study of a special model inspired major advances of the general theory. This was the case, for example, of the exact solutions of the two dimensional Ising and Six-Vertex models in the ambit of the theory of universality; and was certainly the case of the Renormalization Group (RG) analysis of two dimensional Coulomb Gas as prototype of the Kosterlitz-Thouless (KT) transition for systems with long range interactions.

Two dimensional Coulomb Gas is the statistical system of point particles on a plane, carrying a charge \pm 1 , and interacting through the two-dimensional electrostatic potential that, for large distances, is

$$
V(x-y) \sim-\frac{1}{2 \pi} \ln |x-y| .
$$

An ideal realization of the model is a system of infinite, parallel, uniformly charged wires in thermal equilibrium; historically it was proposed as a model of strongly magnetized plasma (see introduction of [15] and references therein). Very soon, anyways, the Coulomb Gas acquired a great theoretical importance, for Berezinskii, [4, and Kosterlitz and Thouless, [26], found in it the solution of a puzzling dichotomy in the theory of the two dimensional $X Y$ model: the spin-wave approximation (quite reliable for low temperatures) predicted, in agreement with the general Mermin-Wagner argument, absence of order and power law fall-off of the spin correlation; on the other hand, high temperature expansion clearly demonstrated exponential decay of the correlations. The two scenarios were merged together 
by the fundamental observation that the former picture did not take into account the spin configurations with vortex excitations, which interacted through the same logarithmic potential written above.

Using RG ideas, Kosterlitz and Thouless, [26], [25], were able to obtain the diagram of phases of the Coulomb Gas (and so of the XY model) depicted in Fig,1, where $z$ is the activity and $\beta$ the inverse temperature. At high temperatures, the gas

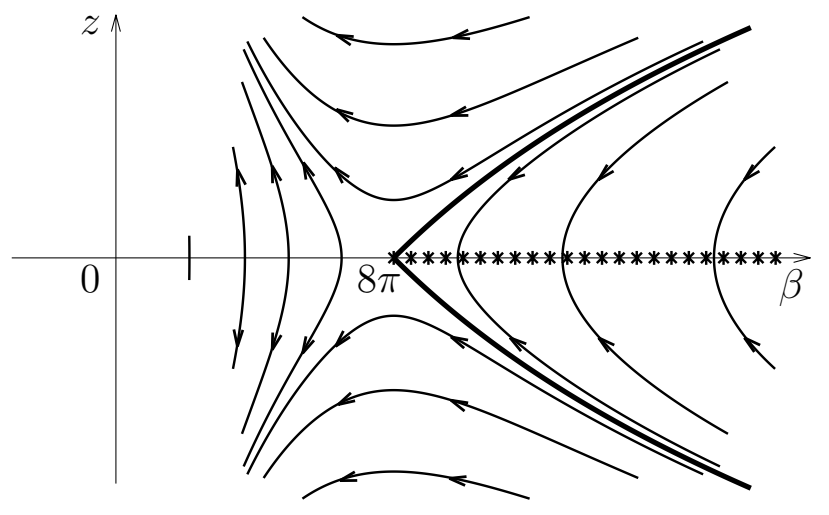

Figure 1: Diagram of phases: the separatrix is the KT line.

is in the plasma phase - or Debye screening phase: the configurations of significant probability are those in which the long-range electrostatic interactions generated by a uniform density of particles of opposite charges almost cancel each others, resulting in an effective, short range potential; as consequence, the correlation length is expected to be finite, and certain screening sum rules are conjectured. Low temperatures, on the contrary, favor the formation of pairs of opposite charges, the dipoles: the effective range of the interactions remains long, and the correlations length is infinite; this regime is the dipole phase - or KT phase. In between the two phases, there is (at least) one critical curve, that is called $K T$ transition line, and was found to intersect the $z=0$ axis at $\beta_{c}(0)=8 \pi,[25]$. This picture represented a new kind of phase transition, because all the temperatures below $\beta_{c}(z)$ are, in a sense, critical; in fact, approaching $\beta_{c}(z)$ from higher temperatures the correlations length is expected to diverge as $\xi \sim \exp \left[c(z)\left|\beta-\beta_{c}(z)\right|^{-\frac{1}{2}}\right]$, as opposed to the $\xi \sim\left|\beta-\beta_{c}\right|^{-\nu}$ (or $\xi \sim \ln \left|\beta-\beta_{c}\right|$ ) of the second-order phase transitions.

After the pioneering analysis of Kosterlitz and Thouless, efforts of many authors were addressed to the topic, in search of stronger evidence of the KT phase transition: the reader interested in theoretical physics works can find useful discussions and a good selection of references in [1], 23] and [15] (see also the conjecture in [20] of an infinite series of KT transition lines, intersecting the $z=0$ axis at $\beta_{c, n}(0)=8 \pi(1-1 / 2 n), n=1,2, \ldots \infty$; and criticism in [34]).

The first major rigorous result was the proof of Fröhlich and Park, [17], of the existence of the thermodynamic limit for pressure and correlations. Later on, 
Fröhlich and Spencer, [18], [19], proved, for $\beta$ large enough, an upper and lower power-law bound for the correlations of fractional (i.e. non-integer) charges; then, refinements of the same technique allowed Marchetti, Klain and Peres, [29], 27] [28], to cover increasing regions in the dipole phase that eventually included the point $(\beta, z)=(8 \pi, 0)$, but not the rest of the KT transition line. Despite its fast improvement, Fröhlich-Spencer method seemed to have some unavoidable limitations: it could not provide the exact power of the correlations fall-off, nor could exclude logarithmic corrections to such decay (which actually were expected along the KT transition line); and it did not provide any useful bound for correlations of integer charges. For this reasons, different authors started developing an RG approach to the model - at the beginning in some approximate form: hierarchical metric, or order by order in perturbation theory; see [2], 30], [12], 33], 24], 33. Later, Dimock and Hurd, [14, achieved a rigorous construction, under no approximation, of the pressure in a region of the dipole phase that included $(\beta, z)=(8 \pi, 0)$ but not the rest of the KT transition line; they could not discuss charge correlations, though. Finally, the only rigorous result on the plasma phase is the work of Yang, [35], that extended to dimension two the proof of the dynamical mass generation for small $\beta$ obtained in [6], [8], for higher dimensional cases.

The objective of this paper is to study the Coulomb Gas along the KT transition line, for small activity. Using the general RG approach of [5] and some model-specific ideas in [14, we are able to give a constructive proof of the existence of the pressure. We shall not discuss here the critical exponents of the correlation functions; but in view of the bounds of this paper, the task should not be difficult and we plan to pursue it in a possible forthcoming work.

Besides, we shall not consider in these pages more complicated models studied by Fröhlich and Spencer, such as the XY, Villain, discrete Gaussian, $Z_{n}$-Clock and the solid-on-solid models, because they require (perhaps) a large-activity framework. Even more interesting - and more difficult to treat - is the surprising (formal) equivalence between the KT transition and the second-order phase transitions of certain two-dimensional, lattice models, such as Ashkin-Teller, Six-Vertex and Eight-Vertex, $Q$-states and anti-ferromagnetic Potts models, $O(n)$ models (including the $n=0$ case, i.e. the Self Avoiding Walks) etc. (see [22], 32] and references therein). Future efforts should be addressed to these appealing applications of the Coulomb Gas.

\section{Definitions and Results}

A possible microscopic realization of the two-dimensional Coulomb Gas is the following. Consider a system of point particles labeled with numbers $1,2,3 \ldots$ and a finite square lattice $\Lambda \subset \mathbb{Z}^{2}$ endowed with periodic boundary conditions: a configuration is the assignment to each of the particles, say the $j$-th, of a charge $\sigma_{j}= \pm 1$ and a position $x_{j} \in \Lambda$. Particles interact through a two-body electrostatic potential 
$W_{\Lambda}\left(x_{i}, x_{j}\right) \equiv W_{\Lambda}\left(x_{i}-x_{j}\right)$, so that, if $\Omega_{n}$ is the set of all the possible configurations of $n$ particles, the total energy (self-energy included) of the configuration $\omega \in \Omega_{n}$ is

$$
H_{\Lambda}(\omega)=\frac{1}{2} \sum_{i, j=1}^{n} \sigma_{i} \sigma_{j} W_{\Lambda}\left(x_{i}-x_{j}\right) .
$$

If $z$ is the activity and $\beta>0$ the inverse temperature, the Grand Canonical partition function is

$$
Z_{\Lambda}(\beta, z)=\sum_{n \geq 0} \frac{z^{n}}{n !} \sum_{\omega \in \Omega_{n}} e^{-\beta H_{\Lambda}(\omega)} .
$$

Before stating the main theorem, we have to give a precise definition of electrostatic potential $W_{\Lambda}$. It is to be the inverse of $-\Delta_{\Lambda}$, the Laplacian operator on $\Lambda$; anyways, because of the periodic boundary conditions on $\Lambda,-\Delta_{\Lambda}$ has zero modes, and we have to assign a regularization procedure to make sense of $\left(-\Delta_{\Lambda}\right)^{-1}$. Define the Yukawa interaction on $\Lambda$ with mass $m$

$$
W_{\Lambda}(x ; m)=\frac{1}{|\Lambda|} \sum_{k \in \Lambda^{*}} \frac{e^{i k x}}{m^{2}-\widehat{\Delta}(k)}
$$

where $\Lambda^{*}$ is the reciprocal lattice of $\Lambda$ and $-\widehat{\Delta}(k)=2 \sum_{j=0,1}\left(1-\cos k_{j}\right)$ is the Fourier transform of $-\Delta_{\Lambda}$; then, if $H_{\Lambda}(\omega ; m)$ is the Yukawa energy of the configuration $\omega$, re-define (2.1) such that

$$
e^{-\beta H_{\Lambda}(\omega)}:=\lim _{m \rightarrow 0} e^{-\frac{\beta}{2} \sum_{i, j=1}^{n} \sigma_{i} \sigma_{j} W_{\Lambda}\left(x_{i}-x_{j} ; m\right)} .
$$

Of course in the massless limit $m \rightarrow 0$ the Yukawa potential, by itself, is ill defined; though we shall see in Sec. A.1 that the above definition makes sense and, in fact, assignes weight zero to configurations of non-neutral total charge - because of that, the system is symmetric under $z \rightarrow-z$. Finally, for $R$ and $L$ positive integers, $L$ odd and bigger than one, assume that $\Lambda$ is a square with a side of $L^{R}$ lattice sites; then the thermodynamic limits of the pressure is

$$
p(\beta, z)=\lim _{R \rightarrow \infty} \frac{1}{\beta|\Lambda|} \ln Z_{\Lambda}(\beta, z) .
$$

The main result of the paper is the following theorem.

Theorem 2.1 For $\varepsilon>0$ small enough, there exists a function $\Sigma(z) \geq \Sigma(0)=8 \pi$ such that, if $|z| \leq \varepsilon$ and $\beta=\Sigma(z)$, the limit (2.5) exists.

As stated at this point, the Theorem is slightly imprecise, because we have not found a way to characterize the Kosterlitz-Thouless transition line in terms of the pressure only; though it will become apparent in the next section that the curve $\beta=\Sigma(z)$ is exactly such line. Lengthier computations, simple but not explicitly pursued in this paper, would prove that $\Sigma(z)$ is a smooth function. 


\section{$3 \quad$ Strategy of the Proof}

The starting point of this analysis is the functional integral representation of the partition function that allows for a more standard RG approach. In Sec. A.1 we shall prove an equivalent formula for the partition function for finite lattice $\Lambda$ :

$$
\begin{array}{r}
Z_{\Lambda}(\beta, z)=\lim _{m \rightarrow 0} \int d P_{\geq R}\left(\zeta^{(R)} ; m\right) \int d P_{R-1}\left(\zeta^{(R-1)}\right) \cdots \int d P_{1}\left(\zeta^{(1)}\right) \\
\cdot \int d P_{0}\left(\zeta^{(0)}\right) e^{\mathcal{V}\left(\zeta^{(0)}+\zeta^{(1)}+\cdots+\zeta^{(R-1)}+\zeta^{(R)}\right)}
\end{array}
$$

where $d P_{\geq R}(\zeta ; m)$ is a Gaussian with massive covariance,

$$
\int d P_{\geq R}(\zeta ; m) \zeta_{x} \zeta_{y}=\Gamma_{\geq R}(x-y ; m),
$$

while $d P_{j}$, for $j=0, \ldots, R-1$, is a Gaussian measure with massless covariance

$$
\int d P_{j}(\zeta) \zeta_{x} \zeta_{y}=\Gamma_{j}(x-y)
$$

Explicit definitions of $\Gamma_{\geq R}$ and $\Gamma_{j}$ (and precise meaning of 'massive' and 'massless') are given in Sec. A.1. Here we stress that, besides being positive-definite functions, $\Gamma_{0}, \Gamma_{1}, \ldots, \Gamma_{R-1}$ satisfy the following properties

$$
\begin{aligned}
& \Gamma_{j}(x)=0 \quad \text { for }|x| \geq L^{j+1} / 2, \\
& \left|\partial^{a} \Gamma_{j}(x)\right| \leq C_{a} L^{-j|a|} \quad \text { for }|x| \leq L^{j+1} / 2, \quad \text { if }|a|>0, \\
& \Gamma_{j}(0)=\frac{1}{2 \pi} \ln L+c_{j}(L) \quad \text { for }\left|c_{j}(L)\right| \leq c L^{-\frac{j}{4}} ;
\end{aligned}
$$

where $C_{a}$ and $c$ are independent of $L$. Namely, $\Gamma_{j}$ has compact support $O\left(L^{j+1}\right)$ and typical momentum $O\left(L^{j}\right)$. Finally, to complete the explanation of (3.1),

$$
\mathcal{V}(\varphi):=|\Lambda| \frac{1}{2} \ln (1-s)+\frac{s}{2} \sum_{\substack{x \in \Lambda \\ \mu \in \overparen{e}}}\left(\partial^{\mu} \varphi_{x}\right)^{2}+z \sum_{\substack{x \in \Lambda \\ \sigma= \pm}} e^{i \sigma \alpha \varphi_{x}},
$$

where $\widehat{e}=\{(1,0),(0,1),(-1,0),(0,-1)\}$ and $\partial^{\mu} \varphi_{x}=\varphi_{x+\mu}-\varphi_{x}$; the notation $\sum_{\mu \in \widehat{e}}$ implies also a factor $1 / 2$ that we do not write explicitly (so that the Fourier transform of $\sum_{\mu \in \widehat{e}} \partial^{-\mu} \partial^{\mu}$ coincides with $\widehat{\Delta}(k)$ defined after (2.3) $)$. The parameter $s$ is in $[0,1 / 2)$ and will be chosen as function of $z$ : in the final limit $R \rightarrow \infty$, it will fix the relation between $\alpha$ and the inverse temperature $\beta$ through the formula $\alpha^{2}=(1-s) \beta$. 
The RG approach consists in computing the integrals in (3.1) progressively from the random variable with highest momentum to the one with lowest. After each integration we define $\mathcal{V}_{j}$, the effective potential on scale $j$, such that $\mathcal{V}_{0}=\mathcal{V}$,

$$
e^{\mathcal{V}_{j+1}(\varphi)}=\int d P_{j}(\zeta) e^{\mathcal{V}_{j}(\varphi+\zeta)}, \quad j=0,1 \ldots, R-1
$$

and, at last,

$$
Z_{\Lambda}(\beta, z)=\lim _{m \rightarrow 0} \int d P_{\geq R}(\zeta ; m) e^{\mathcal{V}_{R}(\zeta)}
$$

In this way the evaluation of the partition function is transformed into the flow of a dynamical system of effective interactions $\mathcal{V}_{0}, \mathcal{V}_{1}, \ldots, \mathcal{V}_{R}$. To have control on it, we must distinguish the irrelevant part of $\mathcal{V}_{j}$, namely the terms that, along the flow, become smaller and smaller by simple 'power counting' arguments, from the relevant part, namely the terms that require a more careful study. In order to do that, it is important to introduce some special kind of lattice domains: blocks and polymers ([5], [10]). Define $|x|:=\max \left\{\left|x_{0}\right|,\left|x_{1}\right|\right\}$. Recall that $L$ was chosen odd; and, for $j=0,1, \ldots, R$, pave the periodic lattice $\Lambda$ with $L^{2(R-j)}$ disjoint squares of size $L^{j}$ in a natural way: there is the central square,

$$
\left\{x \in \Lambda:|x| \leq L^{j} / 2\right\}
$$

and all the other squares are translations of this one by vectors in $L^{j} \mathbb{Z}$. We call these squares $j$-blocks, and we denote the set of $j$-blocks by $\mathcal{B}_{j} \equiv \mathcal{B}_{j}(\Lambda)$. 0-blocks are made of single points, so, for example, $\mathcal{B}_{0}(\Lambda)=\Lambda$. A union of $j$-blocks is called $j$-polymer, and the set of all $j$-polymers in $\Lambda$ is denoted $\mathcal{P}_{j} \equiv \mathcal{P}_{j}(\Lambda)$. Suppose $X$ is a $j$-polymer: $\partial X$ is the set of sites in $X$ with a nearest neighbor outside $X$; $\mathcal{B}_{j}(X)$ is the set of the $j$-blocks in $X ;|X|_{j}$ is the cardinality of $\mathcal{B}_{j}(X)$; and $\bar{X}$ is the smallest polymer in $\mathcal{P}_{j+1}(\Lambda)$ that contains $X$. A polymer made of two blocks, $B, D \in \mathcal{B}_{j}(\Lambda)$, is connected if there exist $x \in B$ and $y \in D$ s.t. $\|x-y\|=1$; the definition extends to connected polymers of more blocks in the usual way. We call $\mathcal{P}_{j}^{c} \equiv \mathcal{P}_{j}^{c}(\Lambda)$ and $\mathcal{S}_{j} \equiv \mathcal{S}_{j}(\Lambda)$ the set of the connected $j$-polymers and the set of the connected $j$-polymers that are made of no more than $4 j$-blocks - the "small polymers" - respectively. $\$_{j} \equiv \$_{j}(\Lambda)$ is the set of the connected polymers that are not small; and $S$ is the ( $j$-independent) number of small $j$-polymers that contain a given $j$-block. Given a $j$-polymer $X$, the collection of its maximal connected parts (each of which is a $j$-polymer by construction) is called $\mathcal{C}_{j}(X)$; while its small set neighborhood is $X^{*}=\cup\left\{Y \in \mathcal{S}_{j}(\Lambda): Y \cap X \neq \emptyset\right\}$. The empty set is considered as an element of $\mathcal{P}_{j}(\Lambda)$, but not of $\mathcal{P}_{j}^{c}(\Lambda)$.

Given a block $B \in \mathcal{B}_{j}$, we define the interaction

$$
U_{j}(s, z, \varphi, B)=V_{j}(s, z, \varphi, B)+W_{j}(s, z, \varphi, B)
$$



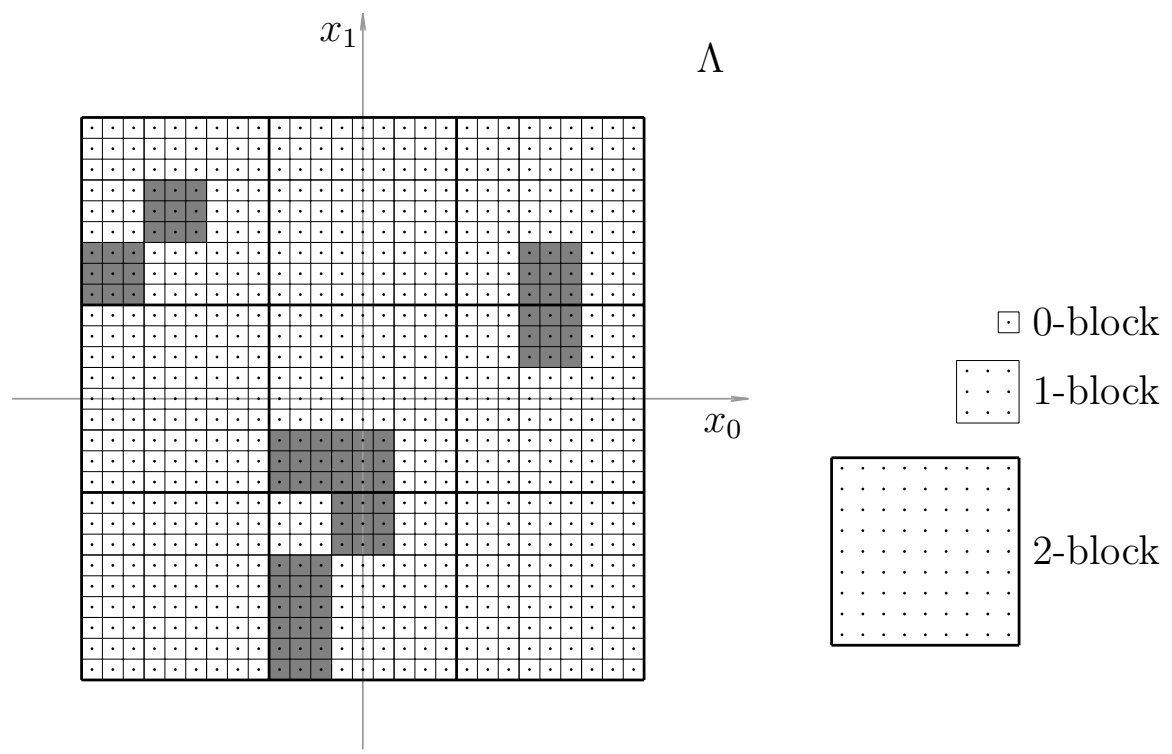

Figure 2: Lattice paving with blocks of different sizes in the case $L=3$ and $R=3$

where $W_{j}(s, z, \varphi, B)$ is quadratic in $s, z$ and will be defined precisely below; while $V_{j}$ is basically given by the original interaction

$$
V_{j}(s, z, \varphi, B)=\frac{s}{2} \sum_{\substack{x \in B \\ \mu \in \widehat{e}}}\left(\partial^{\mu} \varphi_{x}\right)^{2}+z L^{-2 j} \sum_{\substack{x \in B \\ \sigma= \pm}} e^{i \sigma \alpha \varphi_{x}} .
$$

Notice anyways the factor $L^{-2 j}$ that makes $V_{j}$ explicitly $j$-dependent; besides, it actually depends upon $\left\{\varphi_{x}\right\}_{x}$ for $x$ in a domain slightly bigger that $B$ (because includes the outer boundary sites). We assume the $W_{j}(s, z, \varphi, B)$ depends upon $\left\{\varphi_{x}\right\}_{x \in B^{*}}$. We extend these definitions to polymers $X \in \mathcal{P}_{j}$ additively:

$$
U_{j}(s, z, \varphi, X):=\sum_{B \in \mathcal{B}_{j}(X)} U_{j}(s, z, \varphi, B) ;
$$

$V_{j}(s, z, \varphi, X)$ and $W_{j}(s, z, \varphi, X)$ are defined in the same way. If we drop the first two variable of $U_{j}, V_{j}$ and $W_{j}$, it means they are $s_{j}$ and $z_{j}$ - i.e. their have the same label $j$ of the potential. Finally, inductively assume the following formula for the effective potential

$$
\mathcal{V}_{j}(\varphi)=E_{j}|\Lambda|+\ln \left[\sum_{X \in \mathcal{P}_{j}(\Lambda)} e^{U_{j}(\varphi, \Lambda \backslash X)} \prod_{Y \in \mathcal{C}_{j}(X)} K_{j}(\varphi, Y)\right]
$$

where the polymer activity, $K_{j}(\varphi, Y)$, is a function of $\left\{\varphi_{x}\right\}_{x \in Y^{*}}$. For example, the first terms in the sum inside the square brackets are

$$
e^{U_{j}\left(s_{j}, z_{j}, \varphi, \Lambda\right)}+\sum_{Y \in \mathcal{P}_{j}^{c}(\Lambda)} e^{U_{j}\left(s_{j}, z_{j}, \varphi, \Lambda \backslash Y\right)} K_{j}(\varphi, Y)+\cdots
$$


Finally, from (3.7) and (3.11) with $j=R$

$$
Z_{\Lambda}(\beta, z)=e^{E_{R}|\Lambda|} \lim _{m \rightarrow 0} \int d P_{\geq R}(\zeta ; m)\left[e^{U_{R}(\zeta, \Lambda)}+K_{R}(\zeta, \Lambda)\right]:=e^{E_{R+1}|\Lambda|} .
$$

The details of these constructions are in Sec. 4, as opposed to the original RG method in [11], we will not need any 'cluster expansion' to handle the tails of the covariances, for in our setting they have compact support (this idea was devised in [31], [5]). We shall be more specific on the regularity of $U_{j}$ and $K_{j}$ later. At this stage, we just mention that $U_{j}$ is going to contain the second order part of marginal terms of the iteration, whereas the irrelevant terms, as well higher order marginal terms, will be stored in $K_{j}$.

Assumption (3.11) holds at $j=0$, for $\left(E_{0}, s_{0}, z_{0}\right)=\left(\frac{1}{2} \ln (1-s), s, z\right), W_{0} \equiv 0$ and $K_{0} \equiv 0$. In Sec. 4 we shall prove that, iterating (3.6), assumption (3.11) holds at any scale $j=1, \ldots R$, with $\left(s_{j}, z_{j}, K_{j}\right)$ recursively given by: for $j=0$,

$$
\begin{aligned}
& s_{1}=s \\
& z_{1}=L^{2} e^{-\frac{\alpha^{2}}{2} \Gamma_{0}(0)} z \\
& K_{1}=\mathcal{R}_{0}(z, s) ;
\end{aligned}
$$

for $j=1,2, \ldots, R-1$,

$$
\begin{aligned}
& s_{j+1}=s_{j}-a_{j} z_{j}^{2}+\mathcal{F}_{j}\left(K_{j}\right) \\
& z_{j+1}=L^{2} e^{-\frac{\alpha^{2}}{2} \Gamma_{j}(0)}\left[z_{j}-b_{j} s_{j} z_{j}+\mathcal{M}_{j}\left(K_{j}\right)\right] \\
& K_{j+1}=\mathcal{L}_{j}\left(K_{j}\right)+\mathcal{R}_{j}\left(z_{j}, s_{j}, K_{j}\right) .
\end{aligned}
$$

$\mathcal{F}_{j}$ and $\mathcal{M}_{j}$ are real functions of the polymer activity; $\mathcal{L}_{j}$ and $\mathcal{R}_{j}$ are a linear and high order maps of the polymer activities (and functions of $z_{j}$ and $s_{j}$ ). Note that $a_{j} \equiv a_{j}(L), b_{j} \equiv b_{j}(L)$.

Lemma 3.1 If $\alpha^{2}=8 \pi$, set $a:=8 \pi^{2} e^{c} \ln L$ and $b:=2 \ln L$, where $c$ is a constant introduced in (A.32); then there exist $C$ and $C(L)$ such that

$$
\left|L^{2} e^{-\frac{\alpha^{2}}{2} \Gamma_{j}(0)}-1\right| \leq C L^{-\frac{j}{4}}, \quad\left|a_{j}-a\right|,\left|b_{j}-b\right| \leq C(L) L^{-\frac{j}{4}} .
$$

The former inequality is a consequence of (3.4); the latter in proven in Sec. A.3.2. The energy parameters, $E_{0}, E_{1}, \ldots E_{R}$, are recursively defined by

$$
E_{j+1}=E_{j}+L^{-2 j}\left[s_{j}^{2} \widehat{e}_{3, j}+z_{j}^{2} \widehat{e}_{4, j}+s_{j} \widehat{e}_{2, j}+\widehat{e}_{1, j}\left(K_{j}\right)\right],
$$

where $\widehat{e}_{1, j}\left(K_{j}\right)$ is linear in $K_{j}$, while the other $\widehat{e}_{m, j}$ 's are independent of $s_{j}, z_{j}$ and $K_{j} . E_{R+1}$ is defined in (3.12). For $j=0,1, \ldots, R$, let $\mathcal{E}_{j}:=E_{j+1}-E_{j}$. 
Lemma 3.2 There exists $C(\alpha, L)$ such that, for any given $j=0,1, \ldots, R$, if $\left|s_{j}\right|,\left|z_{j}\right|,\left\|K_{j}\right\|_{h, T_{j}} \leq \varepsilon_{0}$,

$$
\left|\mathcal{E}_{j}\right| \leq C(\alpha, L) L^{-2 j} \varepsilon_{0} .
$$

Besides, $\mathcal{E}_{0}, \ldots, \mathcal{E}_{R-1}$ (but not $\mathcal{E}_{R}$ ) are the same on $\Lambda$ and on $\mathbb{Z}^{2}$.

The proof is in Sec. 6.1. (3.14) is the $R G$ map. The solution of (3.14), for initial data $\left(s_{0}, z_{0}, K_{0}\right)=(s, z, 0)$ will be called the $R G$ flow; the sequence of energies given by (3.16) has no influence on the RG flow, hence can be seen as the history of an observable. Consider the case $\alpha^{2}>8 \pi$ : by (3.4) , for $L$ large enough, $L^{2} e^{-\frac{\alpha^{2}}{2} \Gamma_{j}(0)}<$ 1 ; consequently, if also $|z|$ is small enough, the flow goes to zero as was discussed in [14] (in the sub-case of $|z|$ small w.r.t the value of $\beta$ ). This paper is focused on the more complicated case $\alpha^{2}=8 \pi$, when $L^{2} e^{-\frac{\alpha^{2}}{2} \Gamma_{j}(0)}$ is basically 1 and then the flow is determined by the second order terms (see Lemma 3.1). In fact, neglecting higher orders and neglecting the RG map for $\left(K_{j}\right)_{j}$, (3.14) is the equation that Kosterlitz obtained with a (formal) RG technique in coordinate space, [25]. The corresponding approximate solution is, for $j \geq 1$,

$$
s_{j}=\frac{1}{b}\left|q_{j}\right|, \quad z_{j}=\frac{1}{\sqrt{a b}} q_{j},
$$

with $q_{1}$ proportional to $z$ and

$$
q_{j}:=\frac{q_{1}}{1+\left|q_{1}\right|(j-1)} .
$$

(3.18) describes a line, i.e. the first approximation of the separatrix in Fig. E.2, which is the Kosterlitz-Thouless transition line. Our goal is a proof that the solution of the full RG map (3.13), (3.14), is, qualitatively, not too different from (3.18). In order to achieve that, we need to know the smoothness of the remainder terms. In Sec. [5.1, we will set up a norm $\|\cdot\|_{h, T_{j}}$ for polymer activities on scale $j$ depending on two parameters $h$ and $A$ : if properly chosen, the following lemmas hold.

Lemma 3.3 There exist $C, C(\alpha)>1$ such that, if $L$ is large enough,

$$
\left|\mathcal{F}_{j}\left(K_{j}\right)\right| \leq C A^{-1}\left\|K_{j}\right\|_{h, T_{j}}, \quad\left|\mathcal{M}_{j}\left(K_{j}\right)\right| \leq C(\alpha) A^{-1}\left\|K_{j}\right\|_{h, T_{j}} .
$$

Besides, $\mathcal{F}_{1}, \ldots, \mathcal{F}_{R-1}$ and $\mathcal{M}_{1}, \ldots, \mathcal{M}_{R-1}$ are the same on $\Lambda$ and on $\mathbb{Z}^{2}$.

Lemma 3.4 There exist $C(\alpha)>1$ and $\eta, \vartheta>0$ such that, for $L$ large enough,

$$
\left\|\mathcal{L}_{j}\left(K_{j}\right)\right\|_{h, T_{j+1}} \leq C(\alpha)\left(L^{-\vartheta}+A^{-\eta}\right)\left\|K_{j}\right\|_{h, T_{j}} .
$$

Lemma 3.5 If $\varepsilon_{j}>0$ is small enough, there exists $C \equiv C(A, L, \alpha)>1$ such that, for any $\left(s_{j}, z_{j}, K_{j}\right)$ and $\left(\dot{s}_{j}, \dot{z}_{j}, \dot{K}_{j}\right)$ satisfying $\left|s_{j}\right|,\left|z_{j}\right|,\left|\dot{s}_{j}\right|,\left|\dot{z}_{j}\right| \leq \varepsilon_{j}$ and $\left\|K_{j}\right\|_{h, T_{j}},\left\|\dot{K}_{j}\right\|_{h, T_{j}} \leq$ $\varepsilon_{j}^{2}:$ for $j=0$

$$
\left\|\mathcal{R}_{0}(z, s)-\mathcal{R}_{0}(\dot{z}, \dot{s})\right\|_{h, T_{1}} \leq C \varepsilon_{0}[|s-\dot{s}|+|z-\dot{z}|]
$$


while, for $j=1,2, \ldots, R-1$,

$$
\begin{aligned}
& \left\|\mathcal{R}_{j}\left(z_{j}, s_{j}, K_{j}\right)-\mathcal{R}_{j}\left(\dot{z}_{j}, \dot{s}_{j}, \dot{K}_{j}\right)\right\|_{h, T_{j+1}} \\
& \quad \leq C\left[\varepsilon_{j}^{2}\left|s_{j}-\dot{s}_{j}\right|+\varepsilon_{j}^{2}\left|z_{j}-\dot{z}_{j}\right|+\varepsilon_{j}\left\|K_{j}-\dot{K}_{j}\right\|_{h, T_{j}}\right] .
\end{aligned}
$$

The proofs are in Sec. 6. In particular, Lemma 3.4 is crucial: to prove the contraction of $\mathcal{L}_{j}$ we have to show that $\mathcal{L}_{j}\left(K_{j}\right)$ is made of irrelevant terms. The role of constants and parameters so far introduced is the following: $\alpha^{2}=8 \pi$, although in many sub-results of the paper we will just assume $\alpha^{2} \geq 8 \pi ; h$ is a numerical constant related to the propagator, see (5.8); $L$ will be taken large; $A$ is to be large enough w.r.t. $L$; finally, $\varepsilon_{0}$, the size of $z$, is to be small enough w.r.t. $A$ and $L$ (and $\alpha$ ). In a sense, the major improvement w.r.t. [14, crucial for studying the Kosterlitz-Thouless line, is that in this paper $h$ is independent of $L$.

Thanks to Lemma 3.3, 3.4 and 3.5, we have the following result on the RG flow.

Theorem 3.6 Given $L, A$ large enough and an $\varepsilon \equiv \varepsilon(A, L, \alpha)$, in correspondence of any $z,|z| \leq \varepsilon$, there exists an $s=\Sigma(z)$ such that the solution of (3.14) with initial data $(z, s)$ is

$$
s_{j}=\frac{\left|q_{j}\right|}{b}+O\left(j^{-\frac{3}{2}}\right), \quad z_{j}=\frac{q_{j}}{\sqrt{a b}}+O\left(j^{-\frac{3}{2}}\right), \quad\left\|K_{j}\right\|_{h, T_{j}}=O\left(j^{-3}\right),
$$

for $j=0, \ldots, R$ and $q_{1}=O(z)$. Besides, $\Sigma(z)$ can be chosen independent of $\Lambda$.

The proof is given in Sec 7, and uses the fixed point theorem for Banach spaces. Since the flow of $\left(s_{j}, z_{j}, K_{j}\right)$ remains bounded, Lemma 3.2 applies, and

$$
\beta p(\beta, z)=\lim _{R \rightarrow \infty} \frac{\ln Z_{\Lambda}(\beta, z)}{|\Lambda|}=\lim _{R \rightarrow \infty} E_{R+1}=E_{0}+\sum_{j \geq 0} \mathcal{E}_{j},
$$

where the series is convergent because of (3.17) (valid also for $\mathcal{E}_{R+1}$ ). This completes the proof of the main result, Theorem 2.1; in the remaining sections, we shall take up the task of proving all the above sub-results. Note that $C, C(\alpha), C(L, \alpha)$ and $C(A, L, \alpha)$, will indicate (possibly) different values in different equations.

\section{Renormalization Group Map}

In this and the following section we adopt an abridged notation for the fields. In general, we remove the labels $j$ because they will be clear from the context, and label the sum of the fields on higher scales with a prime, so that $\zeta_{x}:=\zeta_{x}^{(j)}$ and $\varphi_{x}^{\prime}:=\zeta_{x}^{(R)}+\zeta_{x}^{(R-1)}+\cdots+\zeta_{x}^{(j+1)}$; besides, $\varphi_{x}:=\varphi_{x}^{\prime}+\zeta_{x}$. We also use $\mathbb{E}_{j}[\cdot]$ for the 
expectations w.r.t. the measure $d P_{j}\left(\zeta^{(j)}\right)$. Therefore the RG map for the effective potential is

$$
e^{\mathcal{V}_{j+1}\left(\varphi^{\prime}\right)}=\mathbb{E}_{j}\left[e^{\mathcal{V}_{j}\left(\varphi^{\prime}+\zeta\right)}\right]
$$

for $\mathcal{V}_{j}(\varphi)$ given by (3.11). As function of the fields, $\mathcal{V}_{0}$ is made of a periodic term of period $2 \pi / \alpha$, and a derivative term: then $\mathcal{V}_{0}$ is invariant under $\varphi_{x} \rightarrow \varphi_{x}+\frac{2 \pi}{\alpha} t$ for the a constant, integer field $t$. The latter property remains true, by induction, for $\mathcal{V}_{j}$ and then for $K_{j}$. As consequence, we shall prove in appendix B the following decomposition of $K_{j}(\varphi, Y)$ into charged components.

Lemma 4.1 For any $j=0,1, \ldots, R$, there exists a decomposition

$$
K_{j}(\varphi, X)=\sum_{q \in \mathbb{Z}} \widehat{K}_{j}(q, \varphi, X)
$$

such that, if $\vartheta$ is a constant field,

$$
\widehat{K}_{j}(q, \varphi+\vartheta, X)=e^{i q \alpha \vartheta} \widehat{K}_{j}(q, \varphi, X)
$$

This simple result is borrowed from [14] and for completeness is reviewed in Sec. B. The 'power counting' argument - that we shall make rigorous in the rest of the paper - implies: a) terms with charge $q$ contract by a factor $L^{-\frac{\alpha^{2}}{4 \pi} q^{2}}$; b) terms proportional to $\left(\partial \varphi^{\prime}\right)^{n}$ contract by a factor $L^{-n} ;$ c) all terms are increased by a volume factor $L^{2}$ (the ratio of volumes of $j+1$ - and $j$-blocks). Therefore, at $\alpha^{2}=8 \pi, \mathrm{RG}$ reduces the size of the components with charge $|q| \geq 2$; of the components with charge $|q|=1$, if the 0-th order Taylor expansion in $\partial \varphi^{\prime}$ has been taken away; and also the of neutral charge component, if the 2-th order Taylor expansion in $\partial \varphi^{\prime}$ has been taken away. The terms so removed are absorbed in $E_{j}, s_{j}, z_{j}$ to give $E_{j+1}, s_{j+1}, z_{j+1}$. Guided by these ideas, we pass to a technical description of RG; because of technical reasons, it is more convenient to define the first RG steps in a different (in fact simpler) way.

\subsection{First RG step}

After integrating the field $\zeta^{(0)}$ in $V_{0}$, we want to recast the effective potential $\mathcal{V}_{1}$ into the form (3.11). There are many ways to achieve this.

Lemma 4.2 Given $s$ and $z$, define $s_{1}$ and $z_{1}$ as in (3.13), and

$$
E_{1}=-\frac{s}{2} \sum_{\mu \in \widehat{e}}\left(\partial^{\mu} \partial^{\mu} \Gamma_{0}\right)^{2}(0) .
$$

There exists a choice of $K_{1}$ and $U_{1}$ such that (3.11) holds with $K_{1}=\mathrm{O}\left(V_{0}^{2}\right)$. 
Proof of Lemma 4.2 - For $D \in \mathcal{B}_{1}$ and $Y \in \mathcal{P}_{1}^{c}$ define

$$
\begin{aligned}
& P_{0}\left(\varphi^{\prime}, \zeta, D\right):=e^{V_{0}(\varphi, D)}-e^{V_{1}\left(\varphi^{\prime}, D\right)+E_{1}|D|}, \\
& P_{0}^{Y}\left(\varphi^{\prime}, \zeta\right):=\prod_{D \in \mathcal{B}_{1}(Y)} P_{0}\left(\varphi^{\prime}, \zeta, D\right) .
\end{aligned}
$$

(recall $V_{0}(\varphi, D) \equiv V_{0}(s, z, \varphi, D)$ and $\left.V_{1}\left(\varphi^{\prime}, D\right) \equiv V_{1}\left(s_{1}, z_{1}, \varphi^{\prime}, D\right)\right)$. Expand formula (3.6) at $j=0$ w.r.t. $P_{0}$, namely

$$
\mathbb{E}_{0}\left[e^{\mathcal{V}_{0}(\varphi, \Lambda)}\right]=\mathbb{E}_{0}\left[\prod_{D \in \mathcal{B}_{1}(\Lambda)}\left(P_{0}\left(\varphi^{\prime}, \zeta, D\right)+e^{V_{1}\left(\varphi^{\prime}, D\right)+E_{1}|D|}\right)\right],
$$

to obtain (3.11) for $U_{1} \equiv V_{1}$ (i.e. $\left.W_{1} \equiv 0\right)$ and

$$
K_{1}\left(\varphi^{\prime}, Y\right)=e^{-E_{1}|Y|} \mathbb{E}_{0}\left[P_{0}^{Y}\left(\varphi^{\prime}, \zeta\right)\right] .
$$

The linear order in $V_{0}$ of (4.7) may not be zero only when $Y$ is a 1 -block $D$ :

$$
\left[\mathcal{L}_{0} K_{0}\right]\left(\varphi^{\prime}, D\right)=\mathbb{E}_{0}\left[V_{0}(\varphi, D)\right]-V_{1}\left(\varphi^{\prime}, D\right)-E_{1}|D|
$$

But this expression is zero by the choice of $s_{1}, z_{1}$ and $E_{1}$.

\section{$4.2 \quad j$-th RG Step}

Consider now $j=1,2, \ldots, R-1$. After integrating the field $\zeta$, we want to recast the effective potential $V_{j+1}$ into the form (3.11):

$$
e^{\mathcal{V}_{j+1}\left(\varphi^{\prime}\right)}=e^{E_{j+1}|\Lambda|} \sum_{X \in \mathcal{P}_{j+1}(\Lambda)} e^{U_{j+1}\left(\varphi^{\prime}, \Lambda \backslash X\right)} \prod_{Y \in \mathcal{C}_{j+1}(X)} K_{j+1}\left(\varphi^{\prime}, Y\right) .
$$

Again, this formula does not determine $K_{j+1}$ and $U_{j+1}$ in a unique way; we want to take advantage of this freedom in order to make $K_{j+1}$ either irrelevant or third order in $V_{j}$. To achieve that, we have to extract a $\bar{Q}_{j}\left(\varphi^{\prime}, X\right)$ from $\mathbb{E}_{j}\left[K_{j}\right]$ and a $Q_{j}\left(\varphi^{\prime}, Y\right)$ from $\mathbb{E}_{j}^{T}\left[V_{j} ; V_{j}\right]$ : the next lemma, purely combinatorial, furnishes such a $K_{j+1}$ and the corresponding $U_{j+1}$.

Lemma 4.3 Suppose to be given two activities: $\bar{Q}_{j}\left(\varphi^{\prime}, X\right)=\mathrm{O}\left(K_{j}\right)$ with support on sets $X \subset \mathcal{S}_{j}$, and $Q_{j}\left(\varphi^{\prime}, Y\right)=\mathrm{O}\left(V_{j}^{2}\right)$ with support on sets $Y \subset \mathcal{S}_{j+1}$; and suppose the difference between $\left(s_{j}, z_{j}, E_{j}\right)$ and $\left(s_{j+1}, z_{j+1}, E_{j+1}\right)$ is such that

$$
\left(E_{j+1}-E_{j}\right)|D|+V_{j+1}\left(\varphi^{\prime}, D\right)-\mathbb{E}_{j}\left[V_{j}(\varphi, D)\right]=\mathrm{O}\left(K_{j}, V_{j}^{2}\right) .
$$

Then there exists a choice of $K_{j+1}$ and $U_{j+1}$ such that (4.9) holds with

$$
K_{j+1}\left(\varphi^{\prime}, Y^{\prime}\right):=\left[\mathcal{L}_{j} K_{j}\right]\left(\varphi^{\prime}, Y^{\prime}\right)+\mathcal{R}_{j}\left(\varphi^{\prime}, Y^{\prime}\right)
$$


where $\mathcal{R}_{j}\left(\varphi^{\prime}, Y^{\prime}\right)=\mathrm{O}\left(K_{j}^{2}, K_{j} V_{j}, V_{j}^{3}\right)$ and,

$$
\begin{aligned}
& {\left[\mathcal{L}_{j} K_{j}\right]\left(\varphi^{\prime}, Y^{\prime}\right)=\sum_{X \in \mathcal{P}_{j}^{c}\left(Y^{\prime}\right)}^{\bar{X}=Y^{\prime}}\left[\mathbb{E}_{j}\left[K_{j}(\varphi, X)\right]-\bar{Q}_{j}\left(\varphi^{\prime}, X\right)\right]} \\
& +\frac{1}{2} \sum_{B_{0}, B_{1} \in \mathcal{B}_{j}\left(Y^{\prime}\right)}^{\overline{B_{0} \cup B_{1}}=Y^{\prime}} \mathbb{E}_{j}^{T}\left[V_{j}\left(\varphi, B_{0}\right) ; V_{j}\left(\varphi, B_{1}\right)\right]-Q_{j}\left(\varphi^{\prime}, Y^{\prime}\right) \\
& -\sum_{D \in \mathcal{B}_{j+1}}^{D=Y^{\prime}}\left[W_{j+1}\left(\varphi^{\prime}, D\right)-\mathbb{E}_{j}\left[W_{j}\left(s_{j+1}, z_{j+1}, \varphi, D\right)\right]-\sum_{Y \in \mathcal{S}_{j+1}}^{Y \supset D} \frac{Q_{j}\left(\varphi^{\prime}, Y\right)}{|Y|_{j+1}}\right] \\
& -\sum_{B \in \mathcal{B}_{j}}^{\bar{B}=Y^{\prime}}\left[\mathcal{E}_{j}|B|+V_{j+1}\left(\varphi^{\prime}, B\right)-\mathbb{E}_{j}\left[V_{j}(\varphi, B)\right]-\sum_{X \in \mathcal{S}_{j}}^{X \supset B} \frac{\bar{Q}_{j}\left(\varphi^{\prime}, X\right)}{|X|_{j}}\right]
\end{aligned}
$$

(recall the short notations for $V_{j}, V_{j+1}$ and $W_{j+1}$ defined after (3.10)).

In the third line there is $W_{j}\left(s_{j+1}, z_{j+1}, \varphi, D\right)$ as opposed to $W_{j}\left(s_{j}, z_{j}, \varphi, D\right)$ : the former is more convenient for our analysis and the difference with the latter is $O\left(V_{j}^{3}, K_{j} V_{j}^{2}, K_{j}^{2}\right)$, which can be left inside $\mathcal{R}_{j}$. Besides, (4.11) gives the third line of (3.14), with $\mathcal{R}_{j}\left(\varphi^{\prime}, Y^{\prime}\right) \equiv\left[\mathcal{R}_{j}\left(s_{j}, z_{j}, K_{j}\right)\right]\left(\varphi^{\prime}, Y^{\prime}\right)$.

Before giving the proof we elaborate on (4.12). As planned before, in the first and second line of the r.h.s. member we read the extraction of $\bar{Q}_{j}$ and $Q_{j}$ from $\mathbb{E}_{j}\left[K_{j}\right]$ and $\mathbb{E}_{j}^{T}\left[V_{j} ; V_{j}\right]$; the same terms are re-absorbed into $E_{j}, s_{j} z_{j}$ in the third line, so obtaining $E_{j+1}, s_{j+1}, z_{j+1}$. Therefore, to determine the latter parameters, we have to choose $\bar{Q}_{j}$ and $Q_{j}$ first.

The choice of $\bar{Q}_{j}$ requires Taylor expansion in $\nabla \varphi^{\prime}$, that we now define. Let $F(\xi, X)$ be a smooth function of the field $\left(\xi_{x}\right)_{x \in X^{*}}$; the $n$-order Taylor expansion of $F(\xi, X)$ at $\xi=0$ is

$$
\left(\operatorname{Tay}_{n} F\right)(\xi, X):=\sum_{m=0}^{n} \frac{1}{m !} \sum_{x_{1} \ldots, x_{m} \in X^{*}} \xi_{x_{1}} \cdots \xi_{x_{m}} \frac{\partial^{m} F}{\partial \xi_{x_{1}} \cdots \partial \xi_{x_{m}}}(0, X) ;
$$

correspondingly, the $n$-order remainder is

$$
\left(\operatorname{Rem}_{n} F\right)(\xi, X):=F(\xi, X)-\left(\operatorname{Tay}_{n} F\right)(\xi, X) .
$$

The Taylor expansion of $K_{j}$ is to be in the field $\xi \sim \nabla \varphi^{\prime}$, namely we have to single out the part of the activity that is purely dipolar. That is accomplished by (4.3): for any point $x_{0} \in X$, if $\left(\delta \varphi^{\prime}\right)_{x}:=\varphi_{x}^{\prime}-\varphi_{x_{0}}^{\prime}$ (which is a sum of $\nabla \varphi^{\prime \prime}$ s),

$$
\widehat{K}_{j}(q, \varphi, X)=e^{i \alpha q \varphi_{x_{0}}^{\prime}} \widehat{K}_{j}\left(q, \delta \varphi^{\prime}+\zeta, X\right) .
$$


Therefore, our choice for $\bar{Q}_{j}$ is

$$
\begin{aligned}
& \bar{Q}_{j}\left(\varphi^{\prime}, X\right)=\frac{1}{|X|} \sum_{x_{0} \in X} \operatorname{Tay}_{2} \mathbb{E}_{j}\left[\widehat{K}_{j}\left(0, \delta \varphi^{\prime}+\zeta, X\right)\right] \\
& +\frac{1}{|X|} \sum_{x_{0} \in X} \sum_{\sigma= \pm 1} e^{i \sigma \alpha \varphi_{x_{0}}^{\prime}} \operatorname{Tay}_{0} \mathbb{E}_{j}\left[\widehat{K}_{j}\left(\sigma, \delta \varphi^{\prime}+\zeta, X\right)\right] \\
& =\mathbb{E}_{j}\left[\widehat{K}_{j}(0, \zeta, X)\right]+\frac{1}{|X|} \sum_{x_{0} \in X} \sum_{\sigma= \pm 1} e^{i \sigma \alpha \varphi_{x_{0}}^{\prime}} \mathbb{E}_{j}\left[\widehat{K}_{j}(\sigma, \zeta, X)\right] \\
& +\frac{1}{|X|} \sum_{\substack{x_{0} \in X \\
x_{1}, x_{2} \in X^{*}}} \mathbb{E}_{j}\left[\frac{\partial^{2} \widehat{K}_{j}}{\partial \varphi_{x_{1}} \partial \varphi_{x_{2}}}(0, \zeta, X)\right] \frac{\left(\varphi_{x_{1}}^{\prime}-\varphi_{x_{0}}^{\prime}\right)\left(\varphi_{x_{2}}^{\prime}-\varphi_{x_{0}}^{\prime}\right)}{2}
\end{aligned}
$$

where Taylor expansions are in $\xi=\delta \varphi^{\prime}$ (the special point $x_{0}$ is averaged over $X$ ); in (4.15) we also used the fact that $K_{j}$ is even in $\zeta$, therefore the expectation of one derivative of $K_{j}$ is zero. We shall prove in Sec. 6.2 that (4.15) makes irrelevant the first line of (4.12). Then, define intermediate parameters $\overline{\mathcal{E}}_{j}=\bar{E}_{j}-E_{j}, \bar{s}_{j}$ and $\bar{z}_{j}$ such that

$$
\begin{aligned}
& \bar{s}_{j}=s_{j}+\mathcal{F}_{j}\left(K_{j}\right), \quad \bar{z}_{j}=L^{2} e^{-\frac{\alpha^{2}}{2} \Gamma_{j}(0)}\left[z+\mathcal{M}_{j}\left(K_{j}\right)\right] \\
& \overline{\mathcal{E}}_{j}=L^{-2 j}\left[\widehat{e}_{1, j}\left(K_{j}\right)+s_{j} \widehat{e}_{2, j}\right]
\end{aligned}
$$

where

$$
\begin{aligned}
& \widehat{e}_{1, j}\left(K_{j}\right)=\sum_{X \in \mathcal{S}_{j}}^{X \ni 0} \frac{1}{|X|_{j}} \mathbb{E}_{j}\left[\widehat{K}_{j}(0, \zeta, X)\right], \quad \widehat{e}_{2, j}=-\frac{L^{2 j}}{2} \sum_{\mu \in \widehat{e}}\left(\partial^{\mu} \partial^{\mu} \Gamma_{j}\right)(0) . \\
& \mathcal{F}_{j}\left(K_{j}\right)=\sum_{X \in \mathcal{S}_{j}}^{X \ni 0} \frac{L^{-2 j}}{|X|_{j}|X|} \sum_{\substack{x_{0} \in X \\
x_{1}, x_{2} \in X^{*}}} \mathbb{E}_{j}\left[\frac{\partial^{2} \widehat{K}_{j}}{\partial \varphi_{x_{1}} \partial \varphi_{x_{2}}}(0, \zeta, X)\right] \sum_{\mu \in \widehat{e}}\left(x_{1}-x_{0}\right)^{\mu}\left(x_{2}-x_{0}\right)^{\mu} \\
& \mathcal{M}_{j}\left(K_{j}\right)=\frac{e^{\frac{\alpha^{2}}{2} \Gamma_{j}(0)}}{2} \sum_{X \in \mathcal{S}_{j}}^{X \ni 0} \frac{1}{|X|_{j}} \sum_{q= \pm 1} \mathbb{E}_{j}\left[\widehat{K}_{j}(q, \zeta, X)\right] .
\end{aligned}
$$

In Sec. 6.2 we shall also prove that, with the above choices, the following quantity (compare it with the third line of (4.12)) is irrelevant:

$$
\sum_{B \in \mathcal{B}_{j}}^{\bar{B}=Y^{\prime}}\left[\overline{\mathcal{E}}_{j}|B|+V_{j+1}\left(\bar{s}_{j}, \bar{z}_{j}, \varphi^{\prime}, B\right)-\mathbb{E}_{j}\left[V_{j}(\varphi, B)\right]-\sum_{X \in \mathcal{S}_{j}}^{X \supset B} \frac{\bar{Q}_{j}\left(\varphi^{\prime}, X\right)}{|X|_{j}}\right]
$$


Then set $Q_{j}$ so that the second line of (4.12) is vanishing:

$$
Q_{j}\left(\varphi^{\prime}, Y^{\prime}\right):=\frac{1}{2} \sum_{B_{0}, B_{1} \in \mathcal{B}_{j}\left(Y^{\prime}\right)}^{\overline{B_{0} \cup B_{1}}=Y^{\prime}} \mathbb{E}_{j}^{T}\left[V_{j}\left(\varphi, B_{0}\right) ; V_{j}\left(\varphi, B_{1}\right)\right] ;
$$

and choose $\mathcal{E}_{j}, s_{j+1}, z_{j+1}$ so that the remaining part of (4.12) vanish:

$$
\begin{aligned}
& \sum_{D \in \mathcal{B}_{j+1}}^{D=Y^{\prime}}\left[\left(\mathcal{E}_{j}-\overline{\mathcal{E}}_{j}\right)|D|+V_{j+1}\left(s_{j+1}-\bar{s}_{j}, z_{j+1}-\bar{z}_{j}, \varphi^{\prime}, D\right)\right. \\
& \left.+W_{j+1}\left(\varphi^{\prime}, D\right)-\mathbb{E}_{j}\left[W_{j}\left(s_{j+1}, z_{j+1}, \varphi, D\right)\right]-\sum_{Y \in \mathcal{S}_{j+1}}^{Y \supset D} \frac{Q_{j}\left(\varphi^{\prime}, Y\right)}{|Y|_{j+1}}\right]=0 .
\end{aligned}
$$

Because of the simple identity

$$
\sum_{Y \in \mathcal{S}_{j+1}}^{Y \supset D} \frac{Q_{j}\left(\varphi^{\prime}, Y\right)}{|Y|_{j+1}}=\frac{1}{2} E^{T}\left[V_{j}\left(s_{j}, z_{j}, \varphi, D\right) ; V_{j}\left(s_{j}, z_{j}, \varphi, D^{*}\right)\right]
$$

and computations in Sec. C.2, cancellation (4.20) is obtained if

$$
\begin{aligned}
& s_{j+1}=\bar{s}_{j}-a_{j} z_{j}^{2}, \quad z_{j+1}=\bar{z}_{j}-L^{2} e^{-\frac{\alpha^{2}}{2} \Gamma_{j}(0)} b_{j} s_{j} z_{j} \\
& \mathcal{E}_{j}=\overline{\mathcal{E}}_{j}+L^{-2 j}\left[s_{j}^{2} \widehat{e}_{3, j}+z_{j}^{2} \widehat{e}_{4, j}\right]
\end{aligned}
$$

and if $W_{j}$ is given by

$$
s^{2} W_{a, j}(\varphi, B)+z^{2}\left[W_{b, j}(\varphi, B)+W_{c, j}(\varphi, B)\right]+z s W_{d, j}(\varphi, B),
$$

where, setting $\Gamma_{j, n}(x):=\Gamma_{n}(x)+\Gamma_{n+1}(x)+\cdots+\Gamma_{j}(x)$ and $f(0 \mid x):=f(0)-f(x)$, the definitions of the functions used in (4.22) and (4.23) are

$$
\begin{gathered}
a_{j}:=\frac{\alpha^{2}}{2} \sum_{y \in \mathbb{Z}}|y|^{2}\left[w_{b, j}(y)\left(e^{-\alpha^{2} \Gamma_{j}(0 \mid y)}-1\right)+e^{-\alpha^{2} \Gamma_{j}(0)}\left(e^{\alpha^{2} \Gamma_{j}(y)}-1\right) L^{-4 j}\right] \\
b_{j}:=\frac{\alpha^{2}}{2} \sum_{\substack{y \in \mathbb{Z} \\
\mu \in \widehat{e}}}\left[\left(\partial^{\mu} \Gamma_{j}\right)^{2}(y)+2 \sum_{n=0}^{j-1}\left(\partial^{\mu} \Gamma_{n}\right)(y)\left(\partial^{\mu} \Gamma_{j}\right)(y) e^{-\frac{\alpha^{2}}{2} \Gamma_{j-1, n}(0)} L^{2(j-n)}\right] \\
\widehat{e}_{3, j}:=\frac{L^{2 j}}{4} \sum_{y \in \mathbb{Z}^{2}} \sum_{\substack{\mu \in \widehat{e} \\
\nu \in \widehat{e}}}\left[\left(\partial^{\mu} \partial^{\nu} \Gamma_{j, 0}\right)(y)+2\left(\partial^{\mu} \partial^{\nu} \Gamma_{j-1,0}\right)(y)\right]\left(\partial^{\mu} \partial^{\nu} \Gamma_{j}\right)(y \mid 0) \\
\widehat{e}_{4, j}:=2 L^{2 j} \sum_{y} w_{b, j}(y)\left[e^{-\alpha^{2} \Gamma_{j}(0 \mid y)}-1-\frac{\alpha^{2}}{2} \sum_{\mu, \nu} \partial^{\mu} \partial^{\nu} \Gamma_{j}(0) y^{\mu} y^{\nu}\right] \\
\quad+L^{-2 j} \sum_{y} e^{-\alpha^{2} \Gamma_{j}(0)}\left(e^{\alpha^{2} \Gamma_{j}(y)}-1\right)
\end{gathered}
$$


and $W_{m, 0}(s, z, \varphi, B)=0$ for $m=a, b, c, d, e$, while for $j \geq 1$,

$$
\begin{aligned}
W_{a, j}(\varphi, B)= & -\sum_{\substack{\mu \in \widehat{e} \\
\nu \in \hat{e}}} \sum_{y \in \mathbb{Z}^{2}} w_{a, j}^{\mu \nu}(y) \sum_{x \in B}\left(\partial^{\mu} \varphi_{x}\right)\left[\left(\partial^{\nu} \varphi_{x+y}\right)-\left(\partial^{\nu} \varphi_{x}\right)\right] \\
W_{b, j}(\varphi, B)= & \sum_{y \in \mathbb{Z}^{2}} w_{b, j}(y) \sum_{\substack{x \in B \\
\sigma= \pm}}\left[e^{i \sigma \alpha\left(\varphi_{x}-\varphi_{x+y}\right)}-1+\frac{\alpha^{2}}{2} \sum_{\mu \nu}\left(\partial^{\mu} \varphi_{x}\right)\left(\partial^{\nu} \varphi_{x}\right) y^{\mu} y^{\nu}\right] \\
W_{c, j}(\varphi, B)= & \sum_{y \in \mathbb{Z}^{2}} w_{c, j}(y) \sum_{\substack{x \in B \\
\sigma= \pm}} e^{i \sigma \alpha\left(\varphi_{x}+\varphi_{x+y}\right)} \\
W_{d, j}(\varphi, B)= & \sum_{\mu} \sum_{y \in \mathbb{Z}^{2}} w_{d, j}^{\mu}(y) \sum_{\substack{x \in B \\
\sigma= \pm}} i \sigma\left[e^{i \sigma \alpha \varphi_{x}}\left(\partial^{\mu} \varphi_{x+y}\right)-e^{i \sigma \alpha \varphi_{x+y}}\left(\partial^{\mu} \varphi_{x}\right)\right] \\
& -\sum_{y \in \mathbb{Z}^{2}} w_{e, j}(y) \sum_{\substack{x \in B \\
\sigma= \pm}}\left(e^{i \sigma \alpha \varphi_{x+y}}-e^{i \sigma \alpha \varphi_{x}}\right)
\end{aligned}
$$

for

$$
\begin{aligned}
& w_{a, j}^{\mu \nu}(y)=\frac{1}{2}\left(\partial^{\mu} \partial^{\nu} \Gamma_{j-1,0}\right)(y) \\
& w_{b, j}(y)=\sum_{n=0}^{j-1} e^{-\alpha^{2} \Gamma_{j-1, n+1}(0 \mid y)} e^{-\alpha^{2} \Gamma_{n}(0)}\left(e^{\alpha^{2} \Gamma_{n}(y)}-1\right) L^{-4 n} \\
& w_{c, j}(y)=\frac{1}{2} \sum_{n=0}^{j-1} e^{-\alpha^{2}\left[\Gamma_{j-1, n+1}(0)+\Gamma_{j-1, n+1}(y)\right]} e^{-\alpha^{2} \Gamma_{n}(0)}\left(e^{-\alpha^{2} \Gamma_{n}(y)}-1\right) L^{-4 n} \\
& w_{d, j}^{\mu}(y)=\frac{\alpha}{2} \sum_{n=0}^{j-1} e^{-\frac{\alpha^{2}}{2} \Gamma_{j-1, n}(0)}\left(\partial^{\mu} \Gamma_{n}\right)(y) L^{-2 n} \\
& w_{e, j}(y)=\frac{\alpha^{2}}{4} \sum_{n=0}^{j-1} e^{-\frac{\alpha^{2}}{2} \Gamma_{j-1, n}(0)} \sum_{\mu}\left[\left(\partial^{\mu} \Gamma_{j-1, n}\right)^{2}(y)-\left(\partial^{\mu} \Gamma_{j-1, n+1}\right)^{2}(y)\right] L^{-2 n}
\end{aligned}
$$

By (3.2),$W_{j}(\varphi, B)$ depends on the field $\varphi_{x}$ for $x$ in a neighborhood of $B$ of diameter $L^{j} / 2$, which is a subset of $B^{*}$. Finally, joining (4.22) with (4.16) we obtain (3.14) and fulfill condition (4.10).

Proof of Lemma 4.3. - The re-blocking operation used here is different from the one in [5], partly because the extraction of $Q_{j}$ (that for the dipoles of [5] is not required), partly because of a different large field regulators introduced below.

Starting from (3.11), reblock the polymers on scale $j+1$ and obtain:

$$
e^{V_{j}(\varphi)}=e^{E_{j}|\Lambda|} \sum_{X \in \mathcal{P}_{j+1}} e^{U_{j}(\varphi, \Lambda \backslash X)} \prod_{Y \in \mathcal{C}_{j+1}(X)} \widetilde{K}_{j}(\varphi, Y)
$$


for

$$
\widetilde{K}_{j}(\varphi, Y):=\sum_{X^{\prime} \in \mathcal{P}_{j}(Y)}^{\overline{X^{\prime}}=Y} e^{U_{j}\left(\varphi, Y \backslash X^{\prime}\right)} \prod_{Y^{\prime} \in \mathcal{C}_{j}\left(X^{\prime}\right)} K_{j}\left(\varphi, Y^{\prime}\right)
$$

If $D$ is a $j+1$-block, and $Z$ a $j+1$-polymer, define

$$
\begin{aligned}
& P_{j}\left(\varphi^{\prime}, \zeta, D\right):=e^{U_{j}(\varphi, D)}-e^{U_{j+1}\left(\varphi^{\prime}, D\right)+\mathcal{E}_{j}|D|}, \\
& P_{j}^{Z}\left(\varphi^{\prime}, \zeta\right):=\prod_{D \in \mathcal{B}_{j+1}(Z)} P_{j}\left(\varphi^{\prime}, \zeta, D\right) ;
\end{aligned}
$$

besides, if $Y$ and $X$ are $j+1$-polymers, define

$$
\begin{aligned}
& R_{j}\left(\varphi^{\prime}, \zeta, Y\right):=\widetilde{K}_{j}(\varphi, Y)-\sum_{D \in \mathcal{B}_{j+1}(Y)} J_{j}\left(\varphi^{\prime}, D, Y\right) \\
& R_{j}^{X}\left(\varphi^{\prime}, \zeta\right):=\prod_{Y \in \mathcal{C}_{j+1}(X)} R_{j}\left(\varphi^{\prime}, \zeta, Y\right)
\end{aligned}
$$

where $J_{j}\left(\varphi^{\prime}, D, Y\right)$ contains the extracted terms $\bar{Q}_{j}$ and $Q_{j}$ (which have support on $j+1$-small and $j$-small polymers, respectively): if $Y \notin \mathcal{S}_{j+1}$, or $D \not \subset Y$, $J_{j}\left(\varphi^{\prime}, D, Y\right) \equiv 0$; otherwise

$$
\begin{aligned}
J_{j}\left(\varphi^{\prime}, D, Y\right) & :=\frac{Q_{j}\left(\varphi^{\prime}, Y\right)}{|Y|_{j+1}}+\sum_{B \in \mathcal{B}_{j}(D)} \sum_{\substack{X \in \mathcal{S}_{j} \\
X \supset B}}^{\bar{X}=Y} \frac{\bar{Q}_{j}\left(\varphi^{\prime}, X\right)}{|X|_{j}} \\
& -\delta_{D, Y} \sum_{Y^{\prime} \in \mathcal{S}_{j+1}}^{Y^{\prime} \supset D}\left[\frac{Q_{j}\left(\varphi^{\prime}, Y^{\prime}\right)}{\left|Y^{\prime}\right|_{j+1}}+\sum_{\substack { B \in \mathcal{B}_{j}(D) \\
\begin{subarray}{c}{X \in \mathcal{S}_{j} \\
X \supset B{ B \in \mathcal { B } _ { j } ( D ) \\
\begin{subarray} { c } { X \in \mathcal { S } _ { j } \\
X \supset B } }\end{subarray}} \frac{\sum_{\substack{X \\
X_{j}}} \bar{Q}_{j}\left(\varphi^{\prime}, X\right)}{|X|_{j}}\right] ;
\end{aligned}
$$

hence $J_{j}\left(\varphi^{\prime}, D, Y\right)$ is a function of $\left\{\varphi_{x}^{\prime}\right\}_{x \in Y^{*}}$; if $Y \notin \mathcal{S}_{j+1}$; and, by construction,

$$
\sum_{Y \in \mathcal{P}_{j+1}^{c}} J_{j}\left(\varphi^{\prime}, D, Y\right)=0
$$

Plugging decompositions (4.29) and (4.30) in (4.27) and expanding we find (4.9), for

$$
\begin{aligned}
K_{j+1}\left(\varphi^{\prime}, Y^{\prime}\right)= & \sum_{\substack{X_{0}, X_{1} \\
Z,(D)}}^{\rightarrow Y^{\prime}} e^{-\mathcal{E}_{j}|W|+U_{j+1}\left(\varphi^{\prime}, Y^{\prime} \backslash W\right)} . \\
& \cdot \mathbb{E}_{j}\left[P_{j}^{Z}\left(\varphi^{\prime}, \zeta\right) R_{j}^{X_{1}}\left(\varphi^{\prime}, \zeta\right)\right] J_{j}^{X_{0},(D)}\left(\varphi^{\prime}\right) .
\end{aligned}
$$


In this formula, we abridged $X_{0} \cup X_{1} \cup Z$ into $W$. And we labeled $\rightarrow Y^{\prime}$ the sum over three $j+1$-polymers, $X_{0}, X_{1}, Z$, and over one $j+1$-block, $D_{Y}$, for each polymer $Y \in \mathcal{C}_{j+1}\left(X_{0}\right)$, such that: a) $X_{0}$ and $X_{1}$ are separated by at least by one $j+1$-block, so that $\mathcal{C}_{j+1}\left(X_{0} \cup X_{1}\right)=\mathcal{C}_{j+1}\left(X_{0}\right)+\mathcal{C}_{j+1}\left(X_{1}\right)$; b) $Z$ is a subset of $Y^{\prime} \backslash\left(X_{0} \cup X_{1}\right)$; c) each connected component of $X_{0}$ is $j+1$-small; d) $\cup_{Y} D_{Y}^{*} \cup Z \cup X_{1}=Y^{\prime}$. Finally, given $X_{0}$ and a $D_{Y}$ for each $Y \in \mathcal{C}_{j+1}\left(X_{0}\right)$, we defined

$$
J_{j}^{X_{0},(D)}\left(\varphi^{\prime}\right):=\prod_{Y \in \mathcal{C}_{j+1}\left(X_{0}\right)} J_{j}\left(\varphi^{\prime}, D_{Y}, Y\right) .
$$

For (4.33) we also used the factorization of $\mathbb{E}_{j}$ over sets that are in two different connected components of a $j+1$-polymer (hence at distance not smaller than $L^{j+1}$, while the range of $\Gamma_{j}$ is $\left.L^{j+1} / 2\right)$. Besides, by construction, $W \subset Y^{\prime}$ so that that $K_{j+1}\left(\varphi^{\prime}, Y\right)$ depends on the fields $\left(\varphi_{y}^{\prime}\right)_{y \in Y^{*}}$ as required.

Finally, we have to prove that the linear part in $K_{j}$ and second order part in $V_{j}$ of this choice of $K_{j+1}$ is (4.12): this follows from two simple identities,

$$
\begin{aligned}
\sum_{D \in \mathcal{B}_{j+1}\left(Y^{\prime}\right)} J_{j}\left(\varphi^{\prime}, D, Y^{\prime}\right)=Q_{j}\left(\varphi^{\prime}, Y^{\prime}\right)+\sum_{X \in \mathcal{S}_{j}}^{\bar{X}=Y^{\prime}} \bar{Q}_{j}\left(\varphi^{\prime}, X\right) \\
-\sum_{D \in \mathcal{B}_{j+1}} \sum_{Y \in \mathcal{S}_{j+1}} \frac{Q_{j}\left(\varphi^{\prime}, Y\right)}{|Y|_{j+1}}-\sum_{B \in \mathcal{B}_{j}} \sum_{X \in \mathcal{S}_{j}}^{\bar{B}=Y^{\prime}} \frac{\bar{Q}_{j}\left(\varphi^{\prime}, X\right)}{|X|_{j}} ;
\end{aligned}
$$

and, by (4.32),

$$
\sum_{Y \in \mathcal{S}_{j+1}} \sum_{D \in \mathcal{B}_{j+1}(Y)}^{D^{*}=Y^{\prime}} J_{j}\left(\varphi^{\prime}, D, Y\right)=\sum_{D \in \mathcal{B}_{j+1}}^{D^{*}=Y^{\prime}} \sum_{Y \in \mathcal{S}_{j+1}}^{Y \supset D} J_{j}\left(\varphi^{\prime}, D, Y\right)=0 .
$$

This completes the proof.

\section{Estimates}

\subsection{Norms and Regulators}

Unless otherwise stated, $j=0,1, \ldots, R-1$. Given $X \in \mathcal{P}_{j}^{c}$, let $\mathcal{C}_{j}^{2}(X)$ be the vector space of the bounded functions $\varphi: X^{*} \rightarrow \mathbb{C}$. For $n=0,1,2$, define (for $\partial^{\mu}$ the discrete derivative defined after (3.5))

$$
\left\|\nabla_{j}^{n} \varphi\right\|_{L^{\infty}(X)}:=\max _{\mu_{1}, \ldots, \mu_{n}} \max _{x \in X} L^{n j}\left|\partial^{\mu_{1}} \ldots \partial^{\mu_{n}} \varphi_{x}\right|
$$

and the norm

$$
\|\varphi\|_{\mathcal{C}_{j}^{2}(X)}:=\max _{n=0,1,2}\left\|\nabla_{j}^{n} \varphi\right\|_{L^{\infty}\left(X^{*}\right)}
$$


Also, consider $L^{2}$ norms:

$$
\begin{aligned}
& \left\|\nabla_{j}^{n} \varphi\right\|_{L_{j}^{2}(X)}^{2}=L^{-2 j} \sum_{x \in X} \sum_{\mu_{1}, \ldots, \mu_{n}} L^{2 n j}\left|\partial^{\mu_{1}} \cdots \partial^{\mu_{n}} \varphi_{x}\right|^{2}, \\
& \left\|\nabla_{j}^{n} \varphi\right\|_{L_{j}^{2}(\partial X)}^{2}=L^{-j} \sum_{x \in \partial X} \sum_{\mu_{1}, \ldots, \mu_{n}} L^{2 n j}\left|\partial^{\mu_{1}} \cdots \partial^{\mu_{n}} \varphi_{x}\right|^{2} .
\end{aligned}
$$

Bounds on expectations of $\mathcal{C}_{j}^{2}$-norms can be obtained by these $L_{j}^{2}$-norms. 1

Let $\mathcal{N}_{j}(X)$ be the space of the smooth complex activities of the polymer $X^{*}$, i.e. the set of $C^{\infty}$ functions $F(\varphi, X): \mathcal{C}_{j}^{2}(X) \rightarrow \mathbb{C}$. The $n$-order derivative of $F$ along the directions $f_{1}, \ldots, f_{n} \in \mathcal{C}_{j}^{2}(X)$ is

$$
D_{\varphi}^{n} F(\varphi, X) \cdot\left(f_{1}, \ldots, f_{n}\right)=\sum_{x_{1}, \ldots, x_{n} \in X^{*}}\left(f_{1}\right)_{x_{1}} \cdots\left(f_{n}\right)_{x_{n}} \frac{\partial^{n} F}{\partial \varphi_{x_{1}} \cdots \partial \varphi_{x_{n}}}(\varphi, X) .
$$

The size of the differential of order $n$ is given by

$$
\|F(\varphi, X)\|_{T_{j}^{n}(\varphi, X)}=\sup _{\left\|f_{i}\right\|_{\mathcal{C}_{j}^{2}(X)} \leq 1}\left|D_{\varphi}^{n} F(\varphi, X) \cdot\left(f_{1}, \ldots, f_{n}\right)\right| .
$$

Then, given any $h>1$, we define the norm

$$
\|F(\varphi, X)\|_{h, T_{j}(\varphi, X)}=\sum_{n \geq 0} \frac{h^{n}}{n !}\|F(\varphi, X)\|_{T_{j}^{n}(\varphi, X)} .
$$

In order to control the norm of the activities as function of the field $\varphi$, for any scale $j$ and any $X \in \mathcal{P}_{j}^{c}$ introduce the field regulators, $G_{j}(\varphi, X) \geq 1$, that depends upon derivatives of $\varphi$ only. Accordingly, define

$$
\|F(X)\|_{h, T_{j}(X)}=\sup _{\varphi \in \mathcal{C}_{j}^{2}(X)} \frac{\|F(\varphi, X)\|_{h, T_{j}(\varphi, X)}}{G_{j}(\varphi, X)} .
$$

Finally, we have to weight the polymer activity w.r.t. the size of the set. Given a parameter $A>1$, define

$$
\|F\|_{h, T_{j}}=\sup _{X \in \mathcal{P}_{j}^{c}} A^{|X|_{j}}\|F(X)\|_{h, T_{j}(X)}
$$

\footnotetext{
${ }^{1}$ By lattice Sobolev lemma $\left((6.136)\right.$ of [5] there exists $c>0$ such that, for any $B \in \mathcal{B}_{j}$,
}

$$
\|\varphi\|_{L^{\infty}(B)}^{2} \leq c \sum_{p=0}^{2}\left\|\nabla_{j}^{p} \varphi\right\|_{L_{j}^{2}(B)}^{2} ;
$$

therefore, for any $X \in \mathcal{P}_{j}^{c}$ and $n=1,2$,

$$
\mathbb{E}_{j}\left[\left\|\nabla_{j}^{n} \zeta^{(j)}\right\|_{L^{\infty}(X)}^{2}\right] \leq C|X|_{j}, \quad \mathbb{E}_{j}\left[\left\|\zeta^{(j)}\right\|_{\mathcal{C}_{j}^{2}(X)}^{2}\right] \leq C|X|_{j} \log L .
$$

The powers $L^{j}$ in definition (5.1) are are chosen to have $C$ independent of $L$ and $j$. 
As already announced, in this paper, as opposed to [14], $h$ is independent $L$ and of the scale $j$ (as well as independent of $\alpha, s$ and $z$ ). Consider the function $h_{j}(x):=$ $\Gamma_{j}(x)-\Gamma_{j}(0)$ : by (3.3), there exists a numerical constant $C_{0}>0$ such that, for any $X \in \mathcal{S}_{j}$ that contains $0,\left\|h_{j}\right\|_{\mathcal{C}_{j}^{2}(X)} \leq C_{0}$; then any fixed $h>1$ that satisfies

$$
h \geq 2 \alpha C_{0}
$$

will work for our purposes.

A convenient choice for the functions $G_{j}$, that guarantees the integrability of the polymer activities at any scale, is inspired by [5]: for $X$ a j-polymer, define

$$
W_{j}(\varphi, X)^{2}:=\sum_{B \in \mathcal{B}_{j}(X)}\|\varphi\|_{L^{\infty}\left(B^{*}\right)}^{2}
$$

then, given two positive constants $c_{1}, c_{3}$, and a positive function of $L, \kappa_{L}$, if $X \in \mathcal{P}_{j}^{c}$

$$
\ln G_{j}(\varphi, X)=c_{1} \kappa_{L}\left\|\nabla_{j} \varphi\right\|_{L_{j}^{2}(X)}^{2}+c_{3} \kappa_{L}\left\|\nabla_{j} \varphi\right\|_{L_{j}^{2}(\partial X)}^{2}+c_{1} \kappa_{L} W_{j}\left(\nabla_{j}^{2} \varphi, X\right)^{2} .
$$

$\mathcal{N}_{j}(X)$ with the norm $\|\cdot\|_{h, T_{j}(X)}$ is a Banach space. For the field dependence of $U_{j}$ we shall use the strong field regulator, $G_{j}^{\text {str }}$ : for $B \in \mathcal{B}_{j}$ and $X \in \mathcal{P}_{j}$,

$$
\ln G_{j}^{\mathrm{str}}(\varphi, B)=\kappa_{L} \max _{n=1,2}\left\|\nabla_{j}^{n} \varphi\right\|_{L^{\infty}\left(B^{*}\right)}^{2}, \quad G_{j}^{\mathrm{str}}(\varphi, X)=\prod_{B \in \mathcal{B}_{j}(X)} G_{j}^{\mathrm{str}}(\varphi, B) .
$$

These definitions suits the Coulomb Gas, more than those ones in [5] (Sec. 6.2.45.), designed for the Dipole Gas; the reason is the need of a finer decomposition of the covariance suggested in [7]; we will discuss this in Sec. D. Anyways the basic structure of the regulators is unchanged, therefore we still refer to [5] in most of the proofs below.

We now list some useful features of the field regulator. As apparent from the definition, if $X \in P_{j+1}$,

$$
G_{j}^{\mathrm{str}}\left(\varphi^{\prime}, X\right) \leq G_{j+1}^{\mathrm{str}}\left(\varphi^{\prime}, X\right) .
$$

For any polymer $X \in \mathcal{P}_{j}$, the sets $Y$ 's in $\mathcal{C}_{j}(X)$ have disjoint boundaries, then the norm $L_{j}^{2}(\partial Y)$ (besides the norm $\left.L_{j}^{2}(Y)\right)$ is additive in $Y$; hence

$$
\prod_{Y \in \mathcal{C}_{j}(X)} G_{j}(\varphi, Y)=G_{j}(\varphi, X) .
$$

In the following results, borrowed or inspired by [5], $c_{3}$ and $c_{1}$ must be large enough, but independent of the scale $j$ and of the parameters $L, s$ and $z$.

Lemma 5.1 For $X \in \mathcal{P}_{j}$,

$$
G_{j}^{\mathrm{str}}(\varphi, X) \leq G_{j}(\varphi, X)
$$

For $X \in \mathcal{P}_{j}$ and $B$ a $j$-block that is not inside $X$,

$$
G_{j}^{\mathrm{str}}(\varphi, B) G_{j}(\varphi, X) \leq G_{j}(\varphi, B \cup X) .
$$


Lemma 5.2 If $\kappa_{L}=c(\log L)^{-1}$ with $c>0$ and small enough:

a) for $X \in \mathcal{P}_{j}^{c}$,

$$
\mathbb{E}_{j}\left[G_{j}(\varphi, X)\right] \leq 2^{|X|_{j}} G_{j+1}\left(\varphi^{\prime}, \bar{X}\right) ;
$$

b) if $X \in \mathcal{S}_{j}$, for a $C>1$,

$$
\left(1+\max _{n=1,2}\left\|\nabla_{j+1}^{n} \varphi^{\prime}\right\|_{L^{\infty}\left(X^{*}\right)}\right)^{3} \mathbb{E}_{j}\left[G_{j}(\varphi, X)\right] \leq C \frac{2^{|X|_{j}}}{\kappa_{L}^{3 / 2}} G_{j+1}\left(\varphi^{\prime}, \bar{X}\right) .
$$

Besides (5.17) holds also if $G_{j}(\varphi, X)$ is replaced by $\sup _{t \in[0,1]} G_{j}\left(t \varphi^{\prime}+\zeta, X\right)$.

For the proof of Lemma 5.1] see Lemma 6.21 and formula (6.52) in [5]; for the former we need $c_{1}>4$, in the latter we have to assume $c_{3}<c c_{1}$, for a certain geometrical constant $c$. Lemma 5.2 has analogies with formulas (6.53) and (6.58) in [5], but the proof is a substantial re-shuffling of the one in that paper, and so is given in Sec. D. It means that $\kappa_{L}=\mathrm{O}(1 / \ln L)$, as opposed to $\kappa_{L}=\mathrm{O}\left(1 / L^{2}\right)$ of [14]: this makes an important difference, because the r.h.s. of (5.17) is then proportional to $\mathrm{O}(\ln L)^{3 / 2}$, and is beaten, for large $L$, by any power of $L$.

Lemma 5.2 holds for $j=0,1, \ldots, R-1$. Let $\mathbb{E}_{R}$ be the expectation with covariance $\Gamma_{\geq R}(x ; m)$, followed by the limit $m \rightarrow 0$.

Lemma 5.3 If $\kappa_{L}=c(\log L)^{-1}$ with $c>0$ and small enough,

$$
\mathbb{E}_{R}\left[G_{R}(\varphi, \Lambda)\right] \leq 2 .
$$

We conclude this section with two useful bounds in [5]. For $\lambda \in(0,1)$, define

$$
k_{s}(A, \lambda)=\sup _{V \in P_{j+1}^{c}} A^{|V|_{j+1}} \sum_{Y \in \mathcal{S}_{j}}^{\bar{Y}=V}(\lambda A)^{-|Y|_{j}}
$$

and

$$
k_{l}(A, \lambda)=\sup _{V \in P_{j+1}^{c}} A^{|V|_{j+1}} \sum_{Y \in \phi_{j}}^{\bar{Y}=V}(\lambda A)^{-|Y|_{j}} .
$$

Lemma 5.4 There exists $c_{s}(\lambda)>0$ such that

$$
k_{s}(A, \lambda) \leq c_{s}(\lambda) L^{2} .
$$

There exist $\eta>0$ and $A_{l}(\lambda, L)$ such that, if $A>A_{l}(\lambda, L)$ then

$$
k_{l}(A, \lambda) \leq A^{-\eta} .
$$

In brief, when the sum is over small sets the bound is proportional to a volume factor $L^{2}$; when the sum is over large sets, the bound is finite in $L$ and vanishing for large $A$. For the proof see Lemma 6.19 and Lemma 6.18 in [5]. 


\subsection{Power Counting Analysis}

For the definition set up so far, we can easily derive some fundamental bounds (mostly introduced in [5]), that will be repeatedly used in the rest of the paper. From the definitions one can easily verify the two following facts: first, for any $\varphi \in \mathcal{C}_{j+1}^{2}(X)$, we have $\|\varphi\|_{\mathcal{C}_{j}^{2}(X)} \leq\|\varphi\|_{\mathcal{C}_{j+1}^{2}(X)}$, so that, for any $F \in \mathcal{N}_{j}(X)$

$$
\|F(\varphi, X)\|_{h, T_{j+1}(\varphi, X)} \leq\|F(\varphi, X)\|_{h, T_{j}(\varphi, X)} ;
$$

second, if $Y \subset X$, for any $\varphi \in \mathcal{C}_{j}^{2}(X)$ we have $\|\varphi\|_{\mathcal{C}_{j}^{2}(Y)} \leq\|\varphi\|_{\mathcal{C}_{j}^{2}(X)}$, so that $\mathcal{C}_{j}^{2}(X) \subset$ $\mathcal{C}_{j}^{2}(Y)$ and

$$
\|F(\varphi, X)\|_{h, T_{j}(\varphi, X)} \leq\|F(\varphi, X)\|_{h, T_{j}(\varphi, Y)} .
$$

For any two polymers $Y_{1}, Y_{2}$ not necessarily disjoint and such that $Y_{1} \cup Y_{2} \subset X$, and any two polymer activities, $F_{1} \in \mathcal{N}_{j}\left(Y_{1}\right)$ and $F_{2} \in \mathcal{N}_{j}\left(Y_{2}\right)$, we have the triangular inequality

$$
\left\|F_{1}\left(\varphi, Y_{1}\right)+F_{2}\left(\varphi, Y_{2}\right)\right\|_{h, T_{j}(\varphi, X)} \leq\left\|F_{1}\left(\varphi, Y_{1}\right)\right\|_{h, T_{j}\left(\varphi, Y_{1}\right)}+\left\|F_{2}\left(\varphi, Y_{2}\right)\right\|_{h, T_{j}\left(\varphi, Y_{2}\right)},
$$

and the factorization property

$$
\left\|F_{1}\left(\varphi, Y_{1}\right) F_{2}\left(\varphi, Y_{2}\right)\right\|_{h, T_{j}(\varphi, X)} \leq\left\|F_{1}\left(\varphi, Y_{1}\right)\right\|_{h, T_{j}\left(\varphi, Y_{1}\right)}\left\|F_{2}\left(\varphi, Y_{2}\right)\right\|_{h, T_{j}\left(\varphi, Y_{2}\right)} .
$$

(The former is a consequence of (5.24) and of the triangular inequality for norms; the latter follows from differentiation rules.) By (5.23) and (5.16), for any $X \in \mathcal{P}_{j}^{c}$

$$
\left\|\mathbb{E}_{j}\left[K_{j}(\varphi, X)\right]\right\|_{h, T_{j+1}\left(\varphi^{\prime}, X\right)} \leq\left\|K_{j}\right\|_{h, T_{j}}\left(\frac{A}{2}\right)^{-|X|_{j}} G_{j+1}\left(\varphi^{\prime}, \bar{X}\right)
$$

and similarly, for each charged component

$$
\left\|\mathbb{E}_{j}\left[\widehat{K}_{j}(q, \varphi, X)\right]\right\|_{h, T_{j+1}\left(\varphi^{\prime}, X\right)} \leq\left\|K_{j}\right\|_{h, T_{j}}\left(\frac{A}{2}\right)^{-|X|_{j}} G_{j+1}\left(\varphi^{\prime}, \bar{X}\right) .
$$

We passed from scale $j+1$ to scale $j$ by the bound (5.23) which is of general validity. But, under special circumstances, this step can be done in a more efficient way and the above bounds can be considerably improved.

Theorem 5.5 If $L$ is large enough, there exists $\vartheta>0$ such that, for any $X \in \mathcal{S}_{j}$,

$$
\left\|\operatorname{Rem}_{2} \mathbb{E}_{j}\left[\widehat{K}_{j}(0, \varphi, X)\right]\right\|\left\|_{h, T_{j+1}\left(\varphi^{\prime}, X\right)} \leq L^{-(2+\vartheta)}\right\| K_{j} \|_{h, T_{j}}\left(\frac{A}{2}\right)^{-|X|_{j}} G_{j+1}\left(\varphi^{\prime}, \bar{X}\right) .
$$

Theorem 5.6 If $L$ is large enough, $\alpha^{2} \geq 8 \pi$ and $|q| \geq 1$, then, for any $X \in \mathcal{S}_{j}$,

$$
\left\|\mathbb{E}_{j}\left[\widehat{K}_{j}(q, \varphi, X)\right]\right\|_{h, T_{j+1}\left(\varphi^{\prime}, X\right)} \leq C(\alpha) L^{-2|q|}\left\|K_{j}\right\|_{h, T_{j}}\left(\frac{A}{2}\right)^{-|X|_{j}} G_{j+1}\left(\varphi^{\prime}, \bar{X}\right) .
$$


Theorem 5.7 If $L$ is large enough, $\alpha^{2} \geq 8 \pi$ and $q= \pm 1$, there exists $\vartheta>0$ such that, for any $X \in \mathcal{S}_{j}$,

$$
\left\|\operatorname{Rem}_{0} \mathbb{E}_{j}\left[\widehat{K}_{j}(q, \varphi, X)\right]\right\|_{h, T_{j+1}\left(\varphi^{\prime}, X\right)} \leq L^{-(2+\vartheta)}\left\|K_{j}\right\|_{h, T_{j}}\left(\frac{A}{2}\right)^{-|X|_{j}} G_{j+1}\left(\varphi^{\prime}, \bar{X}\right)
$$

These results make rigorous power counting arguments mentioned below Lemma 4.1. Theorem [5.6 is borrowed from [14] and means that the charged terms - on small sets - are irrelevant as soon as $L^{-2|q|}$ beats the volume factor $L^{2}$, i.e. for $|q| \geq 2$. Theorem [5.5 is taken from [5] and means that the second order Taylor remainder - again, on small sets only - is an irrelevant term because the factor $L^{-3}(\ln L)^{3 / 2}$ on the r.h.s. member beats the volume factor. Theorem 5.7 is a fusion of the first two: it was missing in [14] and prevented that paper to cover the case of the Kosterlitz-Thouless line. It means that a charged component with $|q|=1$ is irrelevant if the zeroth order Taylor term has been taken away. Although we do not need any essential change respect to the formulations in [14] (even in the case of Theorem 5.7, once the $L$-independence of $h$, found in this paper, is taken into account) we reviewed the proofs of these fundamental results in Sec. E.

\section{Smoothness of RG Map}

\subsection{Proof of Lemma 3.3 and 3.2}

Given $X \in \mathcal{S}_{j}$ and $x_{0} \in X$, set $f_{x}^{\mu}:=\left(x-x_{0}\right)^{\mu}$.

$$
\left|\mathcal{F}_{j}\left(K_{j}\right)\right| \leq C \sum_{X \in \mathcal{S}_{j}}^{X \ni 0} \frac{L^{-2 j}}{|X|_{j}}\left\|\mathbb{E}_{j}\left[\widehat{K}_{j}(0, \zeta, X)\right]\right\|_{h, T_{j+1}\left(\varphi^{\prime}, X\right)} \sum_{\mu \in \widehat{e}}\left\|f^{\mu}\right\|_{\mathcal{C}_{j}^{2}(X)}^{2} ;
$$

as $\left\|f^{\mu}\right\|_{\mathcal{C}_{j}^{2}(X)} \leq C L^{j}$ and by (5.28), (6.1) is bounded by $C A^{-1}\left\|K_{j}\right\|_{h, T_{j}}$.

$$
\left|\mathcal{M}_{j}\left(K_{j}\right)\right| \leq \sum_{X \in \mathcal{S}_{j}}^{X \ni 0} \frac{e^{\frac{\alpha^{2}}{2} \Gamma_{j}(0)}}{|X|_{j}} \sum_{\sigma= \pm 1}\left\|\mathbb{E}_{j}\left[\widehat{K}_{j}(\varepsilon, \zeta, X)\right]\right\|_{h, T_{j+1}\left(\varphi^{\prime}, X\right)},
$$

which, by (3.4) and (5.30), is bounded by $C(\alpha) A^{-1}\left\|K_{j}\right\|_{h, T_{j}}$. Lemma 3.3 is proven.

By (3.2), (3.3), (3.4) and as $\partial^{\mu} \Gamma_{j}(0) \equiv 0$, for $|y| \leq C(L) L^{j}$,

$$
\begin{gathered}
\left|e^{-\alpha^{2} \Gamma_{j}(0 \mid y)}-1-\frac{\alpha^{2}}{2} \sum_{\mu, \nu \in \widehat{e}}\left(\partial^{\mu} \partial^{\nu} \Gamma_{j}\right)(0) y^{\mu} y^{\nu}\right| \leq C(L) L^{-3 j}|y|^{3}, \\
e^{-\alpha^{2} \Gamma_{j}(0)}\left|e^{ \pm \alpha^{2} \Gamma_{j}(y)}-1\right| \leq C(L, \alpha)\left|\Gamma_{j}(y)\right|, \quad\left|e^{-\alpha^{2} \Gamma_{j}(0 \mid y)}-1\right| \leq C(L, \alpha)|y| L^{-j}, \\
\left|\left(\partial^{\mu} \partial^{\nu} \Gamma_{j}\right)(0 \mid y)\right| \leq C(L)|y| L^{-3 j},
\end{gathered}
$$


so that (using also the bounds (

$$
\begin{gathered}
\left|\widehat{e}_{3, j}\right| \leq C(L), \quad\left|\widehat{e}_{2, j}\right| \leq C, \quad\left|\widehat{e}_{4, j}\right| \leq C(L, \alpha), \\
\left|a_{j}\right| \leq C(L, \alpha), \quad\left|b_{j}\right| \leq C(L, \alpha) .
\end{gathered}
$$

Finally

$$
\left|\widehat{e}_{1, j}\left(K_{j}\right)\right| \leq \sum_{X \in \mathcal{S}_{j}}^{X \ni 0} \frac{1}{|X|_{j}}\left\|\mathbb{E}_{j}\left[\widehat{K}_{j}(0, \zeta, X)\right]\right\|_{h, T_{j+1}\left(\varphi^{\prime}, X\right)}
$$

which, by (5.28), is bounded by $C A^{-1}\left\|K_{j}\right\|_{h, T_{j}}$. Finally, a bound for $\mathcal{E}_{R}$ is

$$
\left|\mathcal{E}_{R}\right| \leq \frac{1}{|\Lambda|} \ln \mathbb{E}_{R}\left[1+\left\|e^{U_{R}(\zeta, \Lambda)}-1\right\|_{h, T_{R}(\zeta, \Lambda)}+\left\|K_{R}(\zeta, \Lambda)\right\|_{h, T_{R}(\zeta, \Lambda)}\right] ;
$$

therefore (3.17) with $j=R$ follows from (5.18). Proof of Lemma 3.2 is complete.

\subsection{Proof of Theorem 3.4}

Taking into account cancellation (4.20),

$$
\left(\mathcal{L}_{j} K_{j}\right)\left(\varphi^{\prime}, V\right)=\sum_{p=1}^{5}\left(\mathcal{L}_{j}^{(p)} K_{j}\right)\left(\varphi^{\prime}, V\right)
$$

for (Taylor expansions are in $\delta \varphi^{\prime}$ )

$$
\begin{aligned}
&\left(\mathcal{L}_{j}^{(1)} K_{j}\right)\left(\varphi^{\prime}, V\right):=\sum_{Y \in \mathcal{\phi}_{j}(V)}^{\bar{Y}=V} \mathbb{E}_{j}\left[K_{j}(\varphi, Y)\right] \\
&\left(\mathcal{L}_{j}^{(2)} K_{j}\right)\left(\varphi^{\prime}, V\right):=\sum_{q:|q| \geq 2} \sum_{Y \in \mathcal{S}_{j}(V)}^{\bar{Y}=V} \mathbb{E}_{j}\left[\widehat{K}_{j}(q, \varphi, Y)\right] \\
&\left(\mathcal{L}_{j}^{(3)} K_{j}\right)\left(\varphi^{\prime}, V\right):=\sum_{Y \in \mathcal{S}_{j}(V)} \operatorname{Rem}_{2} \mathbb{E}_{j}\left[\widehat{K}_{j}(0, \varphi, Y)\right] \\
&\left(\mathcal{L}_{j}^{(4)} K_{j}\right)\left(\varphi^{\prime}, V\right):=\sum_{q= \pm 1} \sum_{Y \in \mathcal{S}_{j}(V)} \operatorname{Rem}_{0} \mathbb{E}_{j}\left[\widehat{K}_{j}(q, \varphi, Y)\right] \\
&\left(\mathcal{L}_{j}^{(5)} K_{j}\right)\left(\varphi^{\prime}, V\right):=-\sum_{B \in \mathcal{B}_{j}(V)}^{\bar{B}=V}\left[V_{j+1}\left(\bar{s}_{j}, \bar{z}_{j}, \varphi^{\prime}, B\right)-\mathbb{E}_{j}\left[V_{j}(\varphi, B)\right]\right. \\
&\left.+\overline{\mathcal{E}}_{j}|B|-\sum_{X \in \mathcal{S}_{j}} \frac{\sum^{B}\left(\varphi^{\prime}, X\right)}{|X|_{j}}\right]
\end{aligned}
$$


Consider each term separately: we want to prove that

$$
\left\|\mathcal{L}_{j}^{(p)} K_{j}\right\|_{h, T_{j+1}} \leq \rho(A, L)\left\|K_{j}\right\|_{h, T_{j}}
$$

for $p=1, \ldots, 5$; the constant $\rho(A, L)$ is to be small enough if $L$ and $A$ are large enough, uniformly in the scale $j$.

\subsubsection{Norm of $\mathcal{L}^{(1)}$}

Use (5.25), (5.27) and definition (5.20) to find

$$
\begin{gathered}
\left\|\left(\mathcal{L}_{j}^{(1)} K_{j}\right)\left(\varphi^{\prime}, V\right)\right\|_{h, T_{j+1}\left(\varphi^{\prime}, V\right)} \leq \sum_{Y \in \phi_{j}}^{\bar{Y}=V}\left\|\mathbb{E}_{j}\left[K_{j}(\varphi, Y)\right]\right\|_{h, T_{j+1}\left(\varphi^{\prime}, Y\right)} \\
\leq G_{j+1}\left(\varphi^{\prime}, V\right)\left\|K_{j}\right\|_{h, T_{j}} \sum_{Y \in \phi_{j}}^{\bar{Y}=V} A^{-|Y|_{j}} 2^{|Y|_{j}} \\
\leq G_{j+1}\left(\varphi^{\prime}, V\right) A^{-|V|_{j+1}}\left\|K_{j}\right\|_{h, T_{j}} k_{l}(A, 1 / 2) ;
\end{gathered}
$$

by (5.22), we find (6.11) for $\rho(A, L) \geq A^{-\eta}$.

\subsubsection{Norm of $\mathcal{L}^{(2)}$}

Use (5.30) and definition (5.19) to find

$$
\begin{gathered}
\left\|\left(\mathcal{L}_{j}^{(2)} K_{j}\right)\left(\varphi^{\prime}, V\right)\right\|_{h, T_{j+1}\left(\varphi^{\prime}, V\right)} \leq \sum_{q:|q| \geq 2} \sum_{Y \in \mathcal{S}_{j}(V)}^{\bar{Y}=V}\left\|\mathbb{E}_{j}\left[\widehat{K}_{j}(q, \varphi, Y)\right]\right\|_{h, T_{j+1}\left(\varphi^{\prime}, Y\right)} \\
\leq C(\alpha) G_{j+1}\left(\varphi^{\prime}, V\right) A^{-|V| j+1}\left\|K_{j}\right\|_{h, T_{j}} k_{s}(A, 1 / 2) \sum_{q:|q| \geq 2} L^{-2|q|} ;
\end{gathered}
$$

by (5.21) we obtain (6.11) for $\rho(A, L) \geq C(\alpha) L^{-2}$.

\subsubsection{Norm of $\mathcal{L}^{(3)}$}

By (5.29)

$$
\begin{gathered}
\left\|\left(\mathcal{L}_{j}^{(3)} K_{j}\right)\left(\varphi^{\prime}, V\right)\right\|_{h, T_{j+1}\left(\varphi^{\prime}, V\right)} \leq \sum_{Y \in \mathcal{S}_{j}(V)}^{\bar{Y}=V}\left\|\operatorname{Rem}_{2} \mathbb{E}_{j}\left[\widehat{K}_{j}(0, \varphi, Y)\right]\right\|_{h, T_{j+1}\left(\varphi^{\prime}, Y\right)} \\
\leq G_{j+1}\left(\varphi^{\prime}, V\right) A^{-|V|_{j+1}}\left\|K_{j}\right\|_{h, T_{j}} L^{-(2+\vartheta)} k_{s}(A, 1 / 2)
\end{gathered}
$$

by (5.21) we obtain (6.11) for $\rho(A, L) \geq C L^{-\vartheta}$. 


\subsubsection{Norm of $\mathcal{L}^{(4)}$}

By (5.31),

$$
\begin{gathered}
\left\|\left(\mathcal{L}_{j}^{(4)} K_{j}\right)\left(\varphi^{\prime}, V\right)\right\|_{h, T_{j+1}\left(\varphi^{\prime}, V\right)} \leq \sum_{q= \pm 1} \sum_{Y \in \mathcal{S}_{j}(V)}^{\bar{Y}=V}\left\|\operatorname{Rem}_{0} \mathbb{E}_{j}\left[\widehat{K}_{j}(q, \varphi, Y)\right]\right\|_{h, T_{j+1}\left(\varphi^{\prime}, Y\right)} \\
\leq G_{j+1}\left(\varphi^{\prime}, V\right) A^{-|V|_{j+1}}\left\|K_{j}\right\|_{h, T_{j}} L^{-(2+\vartheta)} k_{s}(A, 1 / 2) .
\end{gathered}
$$

by (5.21) we obtain (6.11) for $\rho(A, L) \geq C L^{-\vartheta}$.

\subsubsection{Norm of $\mathcal{L}^{(5)}$}

Here we prove that (4.18) is irrelevant as claimed before (4.18). Further decompose this term as

$$
\left(\mathcal{L}_{j}^{(5)} K_{j}\right)\left(\varphi^{\prime}, V\right):=\left(\mathcal{L}_{j}^{(5, a)} K_{j}\right)\left(\varphi^{\prime}, V\right)+\left(\mathcal{L}_{j}^{(5, b)} K_{j}\right)\left(\varphi^{\prime}, V\right),
$$

where

$$
\begin{gathered}
\left(\mathcal{L}_{j}^{(5, a)} K_{j}\right)\left(\varphi^{\prime}, V\right)=\sum_{B \in \mathcal{B}_{j}(V)}^{\bar{B}=V}\left[\sum_{X \in \mathcal{S}_{j}} \sum_{x_{0} \in X} \frac{\operatorname{Tay}_{2} \mathbb{E}_{j}\left[\widehat{K}_{j}(0, \varphi, X)\right]}{|X||X|_{j}}\right. \\
\left.-V_{j+1}\left(\mathcal{F}_{j}\left(K_{j}\right), 0, \varphi^{\prime}, B\right)-\widehat{e}_{1, j}\left(K_{j}\right)\right] \\
\left(\mathcal{L}_{j}^{(5, b)} K_{j}\right)\left(\varphi^{\prime}, V\right)=\sum_{B \in \mathcal{B}_{j}(V)}\left[\sum_{X \in \mathcal{S}_{j}}^{\bar{B}=V} \sum_{q= \pm x_{0} \in X} \frac{e^{i q \alpha \varphi_{x_{0}}^{\prime} \mathbb{E}_{j}\left[\widehat{K}_{j}(q, \zeta, X)\right]}}{|X||X|_{j}}\right. \\
\left.-V_{j+1}\left(0, L^{2} e^{-\frac{\alpha^{2}}{2} \Gamma_{j}(0)} \mathcal{M}_{j}\left(K_{j}\right), \varphi^{\prime}, B\right)\right]
\end{gathered}
$$

Consider $\mathcal{L}_{j}^{(5, a)}$. The term proportional to $\widehat{e}_{1, j}$ exactly cancels the one proportional to the zero order of $\operatorname{Tay}_{2} \mathbb{E}_{j}\left[\widehat{K}_{j}(0, \varphi, X)\right]$. We are left with the difference between $V_{j+1}\left(\mathcal{F}_{j}\left(K_{j}\right), 0, \varphi^{\prime}, B\right)$ and the 2 -nd order of $\operatorname{Tay}_{2} \mathbb{E}_{j}\left[\widehat{K}_{j}(0, \varphi, X)\right]$ (the first being zero by symmetry), that, by invariance under space translations of the problem, yields

$$
\left(\mathcal{L}_{j}^{(5, a)} K_{j}\right)\left(\varphi^{\prime}, V\right)=\sum_{B \in \mathcal{B}_{j}(V)}^{\bar{B}=V} \sum_{X \in \mathcal{S}_{j}}^{X \supset B} \sum_{\substack{x_{0} \in X, x_{1}, x_{2} \in X^{*}}} \frac{R_{x_{1}, x_{2}}^{x_{0}}\left(\varphi^{\prime}\right)}{2|X \| X|_{j}} \mathbb{E}_{j}\left[\frac{\partial^{2} \widehat{K}_{j}}{\partial \varphi_{x_{1}} \partial \varphi_{x_{2}}}(0, \zeta, X)\right]
$$


where $R_{x_{1}, x_{2}}^{x_{0}}\left(\varphi^{\prime}\right)$ is given by

$$
\begin{gathered}
\left(\varphi_{x_{2}}^{\prime}-\varphi_{x_{0}}^{\prime}\right)\left(\varphi_{x_{1}}^{\prime}-\varphi_{x_{0}}^{\prime}\right)-L^{-2 j} \sum_{x \in B} \sum_{\mu, \nu \in \widehat{e}}\left(\partial^{\mu} \varphi^{\prime}\right)_{x}\left(\partial^{\nu} \varphi^{\prime}\right)_{x}\left(x_{2}-x_{0}\right)^{\mu}\left(x_{1}-x_{0}\right)^{\nu} \\
=L^{-2 j} \sum_{x \in B}\left[u_{x_{1}, x_{0}}\left(x, \varphi^{\prime}\right) u_{x_{2}, x_{0}}\left(x, \varphi^{\prime}\right)+v_{x_{1}, x_{0}}\left(x, \varphi^{\prime}\right) u_{x_{2}, x_{0}}\left(x, \varphi^{\prime}\right)\right. \\
\left.+u_{x_{1}, x_{0}}\left(x, \varphi^{\prime}\right) v_{x_{2}, x_{0}}\left(x, \varphi^{\prime}\right)\right]
\end{gathered}
$$

and

$$
\begin{aligned}
& u_{x_{1}, x_{0}}(x, \varphi):=\varphi_{x_{1}}-\varphi_{x_{0}}-\sum_{\mu \in \widehat{e}}\left(\partial^{\mu} \varphi\right)_{x}\left(x_{1}-x_{0}\right)^{\mu} \\
& v_{x_{1}, x_{0}}(x, \varphi):=\sum_{\mu \in \widehat{e}}\left(\partial^{\mu} \varphi\right)_{x}\left(x_{1}-x_{0}\right)^{\mu} .
\end{aligned}
$$

Notice that $\left|x_{0}-x_{1}\right|,\left|x_{1}-x_{2}\right| \leq C L^{j}$; it implies

$$
\begin{aligned}
\left\|u_{x_{1}, x_{0}}\left(x, \varphi^{\prime}\right)\right\|_{h, T_{j+1}(\varphi, X)} & \leq C L^{-2}\left(1+\max _{n=1,2}\left\|\nabla_{j+1}^{n} \varphi^{\prime}\right\|_{L^{\infty}\left(X^{*}\right)}\right) \\
\left\|v_{x_{1}, x_{0}}\left(x, \varphi^{\prime}\right)\right\|_{h, T_{j+1}(\varphi, X)} & \leq C L^{-1}\left(1+\max _{n=1,2}\left\|\nabla_{j+1}^{n} \varphi^{\prime}\right\|_{L^{\infty}\left(X^{*}\right)}\right)
\end{aligned}
$$

and then a bound for $\left\|\left(\mathcal{L}_{j}^{(5, a)} K_{j}\right)\left(\varphi^{\prime}, V\right)\right\|_{h, T_{j+1}\left(\varphi^{\prime}, V\right)}$ is

$$
\begin{aligned}
& \leq C\left\|K_{j}\right\|_{h, T_{j}} \sum_{B \in \mathcal{B}_{j}(V)} \sum_{X \in \mathcal{S}_{j}}^{\bar{B}=V}\left(\frac{A}{2}\right)^{-|X|_{j}} \sup _{x_{0}, x_{1}, x_{2}}\left\|R_{x_{0}, x_{1}, x_{2}}^{x_{0}}\left(\varphi^{\prime}\right)\right\|_{h, T_{j+1\left(\varphi^{\prime}, V\right)}} \\
& \leq C^{\prime}\left\|K_{j}\right\|_{h, T_{j}} A^{-|V|_{j+1}} L^{-1}\left(1+\max _{n=1,2}\left\|\nabla_{j+1}^{n} \varphi^{\prime}\right\|_{L^{\infty}\left(V^{*}\right)}^{2}\right) \\
& \leq C^{\prime \prime} \kappa_{L}^{-1} L^{-1}\left\|K_{j}\right\|_{h, T_{j}} G_{j+1}^{\mathrm{str}}\left(\varphi^{\prime}, V\right) A^{-|V|_{j+1}}
\end{aligned}
$$

(the sums over $B$ and $X$ have no more than $C L^{2}$ terms, in all of which $|V|_{j+1}=1$ ). $G_{j+1}^{\text {str }}\left(\varphi^{\prime}, V\right)$ can be replaced by $G_{j+1}\left(\varphi^{\prime}, V\right)$ by (5.14). Next consider $\mathcal{L}_{j}^{(5, b)}$. Simple computations and symmetry $\widehat{K}_{j}(-1, \zeta, X)=\widehat{K}_{j}(1, \zeta, X)$, give

$$
\begin{aligned}
\left(\mathcal{L}_{j}^{(5, b)} K_{j}\right)\left(\varphi^{\prime}, V\right) & =\sum_{B \in \mathcal{B}_{j}(V)}^{\bar{B}=V} L^{-2 j} \sum_{\substack{x_{0} \in B \\
q= \pm 1}} e^{i q \alpha \varphi_{x_{0}}^{\prime}} . \\
& \cdot \sum_{X \in \mathcal{S}_{j}}^{X \supset B} \sum_{y_{0} \in X} \frac{\left(e^{i q \alpha\left(\varphi_{y_{0}}^{\prime}-\varphi_{x_{0}}^{\prime}\right)}-1\right)}{2|X \| X|_{j}} \sum_{\sigma= \pm 1} \mathbb{E}_{j}\left[\widehat{K}_{j}(\varepsilon, \zeta, X)\right] .
\end{aligned}
$$


Notice that by construction $\left|x_{0}-y_{0}\right| \leq C L^{j}$ so that, by (5.14),

$$
\begin{aligned}
\left\|e^{i q \alpha\left(\varphi_{y_{0}}^{\prime}-\varphi_{x_{0}}^{\prime}\right)}-1\right\|_{h, T_{j+1}\left(\varphi^{\prime}, X\right)} & \leq C(\alpha) L^{-1}\left(1+\max _{n=1,2}\left\|\nabla_{j+1}^{n} \varphi^{\prime}\right\|_{L^{\infty}\left(X^{*}\right)}\right) \\
& \leq C^{\prime}(\alpha) \kappa_{L}^{-1} L^{-1} G_{j+1}\left(\varphi^{\prime}, V\right)
\end{aligned}
$$

and then, using (5.30), a bound for $\left\|\left(\mathcal{L}_{j}^{(5, b)} K_{j}\right)\left(\varphi^{\prime}, V\right)\right\|_{h, T_{j+1}\left(\varphi^{\prime}, V\right)}$ is

$$
\begin{gathered}
C(\alpha) \kappa_{L}^{-1} L^{-1} G_{j+1}\left(\varphi^{\prime}, V\right) \sum_{\sigma= \pm} \sum_{B \in \mathcal{B}_{j}(V)}^{\bar{B}=V} \sum_{X \in \mathcal{S}_{j}}^{X \supset B} \frac{1}{|X|_{j}}\left\|\mathbb{E}_{j}\left[\widehat{K}_{j}(\varepsilon, \zeta, X)\right]\right\|_{h, T_{j+1}\left(\varphi^{\prime}, V\right)} \\
\leq C^{\prime}(\alpha) \kappa_{L}^{-1} L^{-1} G_{j+1}\left(\varphi^{\prime}, V\right) A^{-|V|_{j+1}}\left\|K_{j}\right\|_{h, T_{j}}
\end{gathered}
$$

Again we obtain (6.11) for $\rho(A, L) \geq C(\alpha) \kappa_{L}^{-1} L^{-1}$.

\subsection{Preparation Bounds}

We begin with bound (6.74) of [5].

Lemma 6.1 There exists $\eta>0$ such that, for any $X \in P_{j}$,

$$
(1+2 \eta)|\bar{X}|_{j+1} \leq|X|_{j}+8(1+2 \eta)\left|\mathcal{C}_{j}(X)\right|
$$

In the next section we shall need bounds on the "building blocks" of the RG map. Let us introduce a vector notation and a norm with weight $\mu \geq 0$,

$$
\mathcal{K}_{j}:=\left(s_{j}, z_{j}, K_{j}\right), \quad\left|\mathcal{K}_{j}\right|_{\mu, j}:=\mu^{-1}\left|s_{j}\right|+\mu^{-1}\left|z_{j}\right|+\mu^{-2}\left\|K_{j}\right\|_{h, T_{j}} .
$$

Lemma 6.2 There exist $\eta>0, C \equiv C(A, L, \alpha)>1$ such that, for $\varepsilon_{0}$ small enough, 
any $\mathcal{K}_{j}$ and $\dot{\mathcal{K}}_{j}$ with $\left|\mathcal{K}_{j}\right|_{1, j},\left|\dot{\mathcal{K}}_{j}\right|_{1, j} \leq \varepsilon_{0}$ and $D \in \mathcal{B}_{j+1}$ or $Y \in \mathcal{P}_{j+1}^{c}$

$$
\begin{aligned}
& \left\|e^{U_{j}(\varphi, D)}-e^{\dot{U}_{j}(\varphi, D)}\right\|_{h, T_{j}\left(\varphi^{\prime}, D\right)} \leq C \varepsilon_{0}\left|\mathcal{K}_{j}-\dot{\mathcal{K}}_{j}\right|_{\varepsilon_{0}, j} G_{j}^{\mathrm{str}}(\varphi, D) \\
& \left\|e^{U_{j+1}\left(\varphi^{\prime}, D\right)}-e^{\dot{U}_{j+1}\left(\varphi^{\prime}, D\right)}\right\|_{h, T_{j}\left(\varphi^{\prime}, D\right)} \leq C \varepsilon_{0}\left|\mathcal{K}_{j}-\dot{\mathcal{K}}_{j}\right|_{\varepsilon_{0}, j} G_{j+1}^{\mathrm{str}}\left(\varphi^{\prime}, D\right), \\
& \left\|P_{j}\left(\varphi^{\prime}, \zeta, D\right)-\dot{P}_{j}\left(\varphi^{\prime}, \zeta, D\right)\right\|_{h, T_{j}\left(\varphi^{\prime}, D\right)} \\
& \quad \leq C \varepsilon_{0}\left|\mathcal{K}_{j}-\dot{\mathcal{K}}_{j}\right|_{\varepsilon_{0}, j} A^{-(1+\eta)}\left[G_{j+1}^{\mathrm{str}}\left(\varphi^{\prime}, D\right)+G_{j}^{\mathrm{str}}(\varphi, D)\right] \\
& \left\|J_{j}\left(\varphi^{\prime}, D, Y\right)-\dot{J}_{j}\left(\varphi^{\prime}, D, Y\right)\right\|_{h, T_{j}\left(\varphi^{\prime}, Y\right)} \\
& \quad \leq C \varepsilon_{0}^{2}\left|\mathcal{K}_{j}-\dot{\mathcal{K}}_{j}\right|_{\varepsilon_{0}, j} A^{-(1+\eta)\left|D^{*}\right| j+1} G_{j+1}^{\mathrm{str}}\left(\varphi^{\prime}, D\right) \\
& \left\|R_{j}\left(\varphi^{\prime}, \zeta, Y\right)-\dot{R}_{j}\left(\varphi^{\prime}, \zeta, Y\right)\right\|_{h, T_{j}\left(\varphi^{\prime}, Y\right)} \\
& \leq C \varepsilon_{0}^{2}\left|\mathcal{K}_{j}-\dot{\mathcal{K}}_{j}\right|_{\varepsilon_{0}, j} A^{-(1+\eta)|Y|_{j+1}}\left[G_{j+1}^{\mathrm{str}}\left(\varphi^{\prime}, Y\right)+G_{j}(\varphi, Y)\right]
\end{aligned}
$$

where $\dot{U}_{j}, \dot{U}_{j+1}, \dot{P}_{j}, \dot{R}_{j}$ and $\dot{J}_{j}$ contains parameters $\dot{\mathcal{K}}_{j}=\left(\dot{s}_{j}, \dot{z}_{j}, \dot{K}_{j}\right)$ instead of $\mathcal{K}_{j}=\left(s_{j}, z_{j}, K_{j}\right)$.

Remark. Although the $T_{j+1}\left(\varphi^{\prime}, Y\right)$ norm requires a decay in the size of $Y$ as $A^{-|Y|_{j+1}}$, in the r.h.s. of (6.30), (6.31) and (6.32) the decay is (basically) $A^{-(1+\eta)|Y|_{j+1}}$; this is because the extra factor $A^{-\eta|Y|_{j+1}}$ is needed to control the sum in (4.33) (cfr. Sec. 6.4). In (6.30) and (6.31), $|Y|_{j+1}$ is bounded and a $A^{-\eta|Y|_{j+1}}$ factor can be extracted from $C(A, L, \alpha)$; in (6.32), instead, we shall need Lemma 6.1 to have such an improvement.

Proof. Let $B \in \mathcal{B}_{j}(D)$ and use (5.25) and (5.26) to obtain

$$
\begin{aligned}
& \left\|V_{j}(\varphi, B)-\dot{V}_{j}(\varphi, B)\right\|_{h, T_{j}\left(\varphi^{\prime}, B\right)} \leq\left|s_{j}-\dot{s}_{j}\right|\left(h+\left\|\nabla_{j} \varphi\right\|_{L^{\infty}(B)}\right)^{2}+2\left|z_{j}-\dot{z}_{j}\right| e^{\alpha h} \\
& \quad \leq C(\alpha)\left[\left|s_{j}-\dot{s}_{j}\right|+\left|z_{j}-\dot{z}_{j}\right|\right]\left(1+\max _{n=1,2}\left\|\nabla_{j}^{n} \varphi\right\|_{L^{\infty}\left(B^{*}\right)}^{2}\right) .
\end{aligned}
$$


A similar bound holds for $W_{j}$ that, by support property of the propagator, depends on fields at $B^{*}$ (more details are in Appendix C.3):

$$
\begin{aligned}
& \left\|W_{j}(\varphi, B)-\dot{W}_{j}(\varphi, B)\right\|_{h, T_{j}\left(\varphi^{\prime}, B\right)} \\
& \quad \leq C(\alpha)\left[\left|s_{j}-\dot{s}_{j}\right|+\left|z_{j}-\dot{z}_{j}\right|\right] A_{*}^{-1}\left(1+\max _{n=1,2}\left\|\nabla_{j}^{n} \varphi\right\|_{L^{\infty}\left(B^{*}\right)}^{2}\right) .
\end{aligned}
$$

From the definition of $G_{j}^{\text {str }}$

$$
\sum_{B \in \mathcal{B}_{j}(D)}\left(1+\max _{n=1,2}\left\|\nabla_{j}^{n} \varphi\right\|_{L^{\infty}\left(B^{*}\right)}^{2}\right) \leq C(L) G_{j}^{\mathrm{str}}(\varphi, D)^{\frac{1}{3}}
$$

and for $\varepsilon_{0}$ smaller than a $\varepsilon_{0}(L, \alpha)$,

$$
\prod_{B \in \mathcal{B}_{j}(D)} \exp \left\{C(\alpha) \varepsilon_{0}\left(1+\max _{n=1,2}\left\|\nabla_{j}^{n} \varphi\right\|_{L^{\infty}\left(B^{*}\right)}^{2}\right)\right\} \leq 2 G_{j}^{\mathrm{str}}(\varphi, D)^{\frac{1}{3}} .
$$

Then (6.28) follows by factorization property (5.15) and these inequalities. (6.29) is obtained by repeating (6.33) and (6.34) on scale $j+1$; and by taking into account that, by (3.16), (3.14) and the bounds in Sec. 6.1, if $A$ is large enough,

$$
\max \left\{\left|\mathcal{E}_{j}-\dot{\mathcal{E}}_{j}\right| L^{2 j+2},\left|s_{j+1}-\dot{s}_{j+1}\right|,\left|z_{j+1}-\dot{z}_{j+1}\right|\right\} \leq C(L, \alpha)\left|\mathcal{K}_{j}-\dot{\mathcal{K}}_{j}\right|_{1, j}
$$

Accordingly, for $\varepsilon_{0}$ smaller than a $\varepsilon_{0}(L, \alpha)$,

$$
\begin{array}{r}
\left\|e^{U_{j+1}\left(\varphi^{\prime}, D\right)+\mathcal{E}_{j}|D|}-e^{\dot{U}_{j+1}\left(\varphi^{\prime}, D\right)+\dot{\mathcal{E}}_{j}|D|}\right\|_{h, T_{j}\left(\varphi^{\prime}, D\right)} \\
\leq C(L, \alpha)\left|\mathcal{K}_{j}-\dot{\mathcal{K}}_{j}\right|_{1, j} G_{j}^{\mathrm{str}}\left(\varphi^{\prime}, D\right),
\end{array}
$$

which, together to (6.28), proves (6.30). Next, consider (6.31). By (4.15), for any $X \in \mathcal{S}_{j}$ that contains a given $B \in \mathcal{B}_{j}(D)$ (if $L>8$ then $X^{*} \subset D^{*}$ )

$$
\left\|\bar{Q}_{j}\left(\varphi^{\prime}, X\right)-\dot{\bar{Q}}_{j}\left(\varphi^{\prime}, X\right)\right\|_{h, T_{j}\left(\varphi^{\prime}, X\right)} \leq C(\alpha) A^{-|X|_{j}}\|K-\dot{K}\|_{h, T_{j}} G_{j+1}^{\mathrm{str}}\left(\varphi^{\prime}, D\right)
$$

then, as $\left|D^{*}\right| \leq 4 S$, replace $C(\alpha) A^{-|X|_{j}}$ with $C(A, \alpha) A^{-(1+\eta)\left|D^{*}\right|_{j+1}}$. Besides, for any $Y \in \mathcal{S}_{j+1}$ that contains a given block $D \in \mathcal{B}_{j+1}$, by the second order computation in in Appendix C.2.

$$
\begin{aligned}
& \left\|Q_{j}\left(\varphi^{\prime}, Y\right)-\dot{Q}_{j}\left(\varphi^{\prime}, Y\right)\right\|_{h, T_{j}\left(\varphi^{\prime}, Y\right)} \\
& \leq C(L, \alpha) \varepsilon_{0}\left[\left|s_{j}-\dot{s}_{j}\right|+\left|z_{j}-\dot{z}_{j}\right|\right] G_{j+1}^{\mathrm{str}}\left(\varphi^{\prime}, D\right)
\end{aligned}
$$

again we replace $C(L, \alpha)$ with $C(A, L, \alpha) A^{-(1+\eta)\left|D^{*}\right|_{j+1}}$ as $\left|D^{*}\right|_{j+1} \leq 4 S$. Together, (6.39) and (6.40) prove (6.31). Bounds so far have been straightforward consequences of the properties of the norms. As announced, bound (6.32) is slightly 
more sophisticated because requires Lemma 6.1.

$$
\begin{gathered}
\widetilde{K}_{j}(\varphi, Y)-\dot{\widetilde{K}}_{j}(\varphi, Y)=\sum_{X \in \mathcal{P}_{j}^{c}}^{\bar{X}=Y}\left[U_{j}(\varphi, Y \backslash X)-\dot{U}_{j}(\varphi, Y \backslash X)\right] \prod_{Z \in \mathcal{C}_{j}(X)} K_{j}(\varphi, Z) . \\
\cdot \int_{0}^{1} d t\left[e^{t U_{j}(\varphi, Y \backslash X)+(1-t) \dot{U}_{j}(\varphi, Y \backslash X)}\right] \\
+\sum_{X \in \mathcal{P}_{j}^{c}} e^{\dot{U}_{j}(\varphi, Y \backslash X)} \sum_{X_{0} \in((X))_{j}} \prod_{Z \in \mathcal{C}_{j}\left(X_{0}\right)}\left[K_{j}(\varphi, Z)-\dot{K}_{j}(\varphi, Z)\right] \prod_{Z \in \mathcal{C}_{j}\left(X \backslash X_{0}\right)} \dot{K}_{j}(\varphi, Z)
\end{gathered}
$$

where $((X))_{j}$ is the family of sets made of unions of connected parts of $X$, empty set excluded. Therefore, using the factorization property (5.15) and previous bounds

$$
\begin{aligned}
& \left\|\widetilde{K}_{j}(\varphi, Y)-\dot{\widetilde{K}}_{j}(\varphi, Y)\right\|_{h, T_{j}(\varphi, Y)} \\
& \leq\left[\left|s_{j}-\dot{s}_{j}\right|+\left|z_{j}-\dot{z}_{j}\right|\right] G_{j}(\varphi, Y) \sum_{X \in \mathcal{P}_{j}^{c}}^{\bar{X}=Y} C(\alpha)^{|Y \backslash X|_{j}} A^{-|X|_{j}} \varepsilon_{0}^{\left|\mathcal{C}_{j}(X)\right|} \\
& \quad+\varepsilon_{0}^{-1}\left\|K_{j}-\dot{K}_{j}\right\|_{h, T_{j}} G_{j}(\varphi, Y) \sum_{X \in \mathcal{P}_{j}^{c}}^{\bar{X}=Y} 2^{|Y \backslash X|_{j}} 4^{\left|C_{j}(X)\right|} A^{-|X|_{j}} \varepsilon_{0}^{\left|\mathcal{C}_{j}(X)\right|} \\
& \leq \varepsilon_{0}\left|\mathcal{K}_{j}-\dot{\mathcal{K}}_{j}\right|_{\varepsilon_{0}, j} G_{j}(\varphi, Y) C^{\prime}(\alpha)^{|Y|_{j}} \sum_{X \in \mathcal{P}_{j}^{c}}^{\bar{X}=Y} A^{-|X|_{j}}\left(4 \varepsilon_{0}\right)^{\left|\mathcal{C}_{j}(X)\right|}
\end{aligned}
$$

In order to extract the factor $A^{-(1+\eta)|Y|_{j+1}}$ and, at the same time, to control the sum over $X$, which is made of no more than $2^{|Y|_{j}}=2^{L^{2}|Y|_{j+1}}$ terms, use (6.26) and obtain

$$
A^{-|X|_{j}} A^{-8(1+2 \eta)\left|C_{j}(X)\right|} \leq A^{-(1+2 \eta)|Y|_{j+1}}
$$

Therefore, for $4 A^{8(1+2 \eta)} \varepsilon_{0}<1$, bound (6.42) is smaller than

$$
A^{-(1+\eta)|Y|_{j+1}} \varepsilon_{0}\left|\mathcal{K}_{j}-\dot{\mathcal{K}}_{j}\right|_{\varepsilon_{0}, j} G_{j}(\varphi, Y)\left(C(\alpha)^{L^{2}} A^{-\eta}\right)^{|Y|_{j+1}} 4 A^{8(1+2 \eta)} \varepsilon_{0}
$$

finally, for $A$ large enough, $C(\alpha)^{L^{2}} A^{-\eta} \leq 1$ and choosing $C(A, \alpha, L) \geq 4 A^{8(1+2 \eta)}$,

$$
\begin{aligned}
\| \widetilde{K}_{j}(\varphi, Y) & -\dot{\widetilde{K}}_{j}(\varphi, Y) \|_{h, T_{j}\left(\varphi^{\prime}, Y\right)} \\
\leq & \leq C(A, L, \alpha) A^{-(1+\eta)|Y|_{j+1}} \varepsilon_{0}^{2}\left|\mathcal{K}_{j}-\dot{\mathcal{K}}_{j}\right|_{\varepsilon_{0}, j} G_{j}(\varphi, Y) .
\end{aligned}
$$

To conclude, (6.32) follows from (6.31) and (6.45). This completes the proof. 


\subsection{Proof of Theorem 3.5}

We need an explicit formula for $\mathcal{R}_{j}\left(s_{j}, z_{j}, K_{j}\right)$. Expanding (4.33) we obtain:

$$
\begin{gathered}
K_{j+1}\left(\varphi^{\prime}, Y^{\prime}\right)=\mathbb{E}_{j}\left[P_{j}^{Y^{\prime}}\left(\varphi^{\prime}, \zeta\right)\right]+\left(e^{-\mathcal{E}_{j}\left|Y^{\prime}\right|}-1\right) \mathbb{E}_{j}\left[P_{j}^{Y^{\prime}}\left(\varphi^{\prime}, \zeta\right)\right] \\
+\sum_{\mathcal{C}_{j+1}\left(X_{0} \cup X_{1}\right) \geq 1}^{\rightarrow Y^{\prime}} \mathbb{E}_{j}\left[P_{j}^{Z}\left(\varphi^{\prime}, \zeta\right) R_{j}^{X_{1}}\left(\varphi^{\prime}, \zeta\right)\right] J_{j}^{X_{0},(D)}\left(\varphi^{\prime}\right) \\
+\sum_{\mathcal{C}_{j+1}\left(X_{0} \cup X_{1}\right) \geq 1}\left(e^{-\mathcal{E}_{j}|W|+U_{j+1}\left(\varphi^{\prime}, W \backslash Y^{\prime}\right)}-1\right) \cdot \\
\cdot \mathbb{E}_{j}\left[P_{j}^{Z}\left(\varphi^{\prime}, \zeta\right) R_{j}^{X_{1}}\left(\varphi^{\prime}, \zeta\right)\right] J_{j}^{X_{0},(D)}\left(\varphi^{\prime}\right) .
\end{gathered}
$$

Next, we have to remove the part $\mathcal{L}_{j} K_{j}$ : to this purpose, further expand the first term in the r.h.s. of (6.46)

$$
\begin{aligned}
\sum_{D \in \mathcal{B}_{j+1}}^{D=Y^{\prime}} \mathbb{E}_{j}\left[P_{j}\left(\varphi^{\prime}, \zeta, D\right)\right]+ & \frac{1}{2} \sum_{\substack{D_{0}, D_{1} \in \mathcal{B}_{j+1} \\
D_{0} \neq D_{1}}}^{D_{0} \cup D_{1}=Y^{\prime}} \mathbb{E}_{j}\left[P_{j}\left(\varphi^{\prime}, \zeta, D_{0}\right) P_{j}\left(\varphi^{\prime}, \zeta, D_{1}\right)\right] \\
& +\sum_{\substack{Z \in P_{j+1} \\
|Z|_{j+1} \geq 3}}^{Z=Y^{\prime}} \mathbb{E}_{j}\left[P_{j}^{Z}\left(\varphi^{\prime}, \zeta\right)\right] ;
\end{aligned}
$$

and also further expand the second line of the r.h.s. member of (6.46)

$$
\begin{aligned}
& \mathbb{E}_{j}\left[R_{j}\left(\varphi^{\prime}, \zeta, Y^{\prime}\right)\right]+\sum_{Y \in \mathcal{S}_{j+1}} \sum_{D \in \mathcal{B}_{j+1}(Y)}^{D=Y^{\prime}} J_{j}\left(\varphi^{\prime}, D, Y\right) \\
& +\sum_{\substack{\mathcal{C}_{j+1}\left(X_{0} \cup X_{1}\right) \geq 1 \\
|Z|_{j+1}+\mathcal{C}_{j+1}\left(X_{0} \cup X_{1}\right) \geq 2}}^{\rightarrow Y^{\prime}} \mathbb{E}_{j}\left[P_{j}^{Z}\left(\varphi^{\prime}, \zeta\right) R_{j}^{X_{1}}\left(\varphi^{\prime}, \zeta\right)\right] J_{j}^{X_{0},(D)}\left(\varphi^{\prime}\right) .
\end{aligned}
$$

Grouping together the above decompositions and definition (4.12), we obtain

$$
\mathcal{R}_{j}\left(\varphi^{\prime}, Y^{\prime}\right):=K_{j+1}\left(\varphi^{\prime}, Y^{\prime}\right)-\left(\mathcal{L}_{j} K_{j}\right)\left(\varphi^{\prime}, Y^{\prime}\right)=\sum_{s=1}^{6} \mathcal{R}_{j}^{(s)}\left(\varphi^{\prime}, Y^{\prime}\right)
$$


where the eight summands are

$$
\begin{aligned}
& \mathcal{R}_{j}^{(1)}\left(\varphi^{\prime}, Y^{\prime}\right):=\sum_{D \in \mathcal{B}_{j+1}}^{D=Y^{\prime}}\left[\mathbb{E}_{j}\left[P_{j}\left(\varphi^{\prime}, \zeta, D\right)\right]+\mathcal{E}_{j}|D|+U_{j+1}\left(\varphi^{\prime}, D\right)\right. \\
& \left.-\mathbb{E}_{j}\left[U_{j}(\varphi, D)\right]-\frac{1}{2} \mathbb{E}_{j}^{T}\left[V_{j}(\varphi, D) ; V_{j}(\varphi, D)\right]\right] \\
& \mathcal{R}_{j}^{(2)}\left(\varphi^{\prime}, Y^{\prime}\right):=\sum_{D \in \mathcal{B}_{j+1}}^{D=Y^{\prime}}\left[\mathbb{E}_{j}\left[W_{j}\left(s_{j+1}, z_{j+1}, \varphi, D\right)\right]-\mathbb{E}_{j}\left[W_{j}\left(s_{j}, z_{j}, \varphi, D\right)\right]\right] \\
& \mathcal{R}_{j}^{(3)}\left(\varphi^{\prime}, Y^{\prime}\right):=\frac{1}{2} \sum_{\substack{D_{0}, D_{1} \in \mathcal{B}_{j+1} \\
D_{0} \neq D_{1}}}^{D_{0} \cup D_{1}=Y^{\prime}}\left[\mathbb{E}_{j}\left[P_{j}\left(\varphi^{\prime}, \zeta, D_{0}\right) P_{j}\left(\varphi^{\prime}, \zeta, D_{1}\right)\right]\right. \\
& \left.-\mathbb{E}_{j}^{T}\left[V_{j}\left(\varphi, D_{0}\right) ; V_{j}\left(\varphi, D_{1}\right)\right]\right] \\
& \mathcal{R}_{j}^{(4)}\left(\varphi^{\prime}, Y^{\prime}\right):=\sum_{\substack{\mathcal{C}_{j+1}\left(X_{0} \cup X_{1}\right) \geq 1 \\
|Z|_{j+1}+\mathcal{C}_{j+1}\left(X_{0} \cup X_{1}\right) \geq 2}}^{\rightarrow Y^{\prime}} \mathbb{E}_{j}\left[P_{j}^{Z}\left(\varphi^{\prime}, \zeta\right) R_{j}^{X_{1}}\left(\varphi^{\prime}, \zeta\right)\right] J_{j}^{X_{0},(D)}\left(\varphi^{\prime}\right) \\
& \mathcal{R}_{j}^{(5)}\left(\varphi^{\prime}, Y^{\prime}\right):=\sum_{\mathcal{C}_{j+1}\left(X_{0} \cup X_{1}\right) \geq 1}^{\rightarrow Y^{\prime}}\left(e^{-\mathcal{E}_{j}|W|+U_{j+1}\left(\varphi^{\prime}, Y^{\prime} \backslash W\right)}-1\right) . \\
& \cdot \mathbb{E}_{j}\left[P_{j}^{Z}\left(\varphi^{\prime}, \zeta\right) R_{j}^{X_{1}}\left(\varphi^{\prime}, \zeta\right)\right] J_{j}^{X_{0},(D)}\left(\varphi^{\prime}\right) \\
& \mathcal{R}_{j}^{(6)}\left(\varphi^{\prime}, Y^{\prime}\right):=\left(e^{-\mathcal{E}_{j}\left|Y^{\prime}\right|}-1\right) \mathbb{E}_{j}\left[P_{j}^{Y^{\prime}}\left(\varphi^{\prime}, \zeta\right)\right]+\sum_{\substack{Z \in P_{j+1} \\
|Z|_{j+1} \geq 3}}^{Z=Y^{\prime}} \mathbb{E}_{j}\left[P_{j}^{Z}\left(\varphi^{\prime}, \zeta\right)\right] \\
& \mathcal{R}_{j}^{(7)}\left(\varphi^{\prime}, Y^{\prime}\right):=\sum_{\substack{X^{\prime} \in P_{j} \\
\mathcal{C}_{j}\left(X^{\prime}\right) \geq 2}}^{\overline{X^{\prime}}=Y^{\prime}} \mathbb{E}_{j}\left[e^{U_{j}\left(\varphi, Y^{\prime} \backslash X^{\prime}\right)} \prod_{Y \in \mathcal{C}_{j}\left(X^{\prime}\right)} K_{j}(\varphi, Y)\right] \\
& \mathcal{R}_{j}^{(8)}\left(\varphi^{\prime}, Y^{\prime}\right):=\sum_{X^{\prime} \in P_{j}}^{\overline{X^{\prime}}=Y^{\prime}} \mathbb{E}_{j}\left[\left(e^{U_{j}\left(\varphi, Y^{\prime} \backslash X^{\prime}\right)}-1\right) \prod_{Y \in \mathcal{C}_{j}\left(X^{\prime}\right)} K_{j}(\varphi, Y)\right] .
\end{aligned}
$$


Notice that, for $j=0$, we instead have

$$
\begin{aligned}
\mathcal{R}_{0}\left(\varphi^{\prime}, Y^{\prime}\right):= & \sum_{D \in \mathcal{B}_{1}}^{D=Y^{\prime}}\left[\mathbb{E}_{0}\left[P_{0}\left(\varphi^{\prime}, \zeta, D\right)\right]+\mathcal{E}_{1}|D|+V_{1}\left(\varphi^{\prime}, D\right)-\mathbb{E}_{1}\left[V_{1}(\varphi, D)\right]\right] \\
& +\left(e^{-\mathcal{E}_{1}\left|Y^{\prime}\right|}-1\right) \mathbb{E}_{j}\left[P_{0}^{Y^{\prime}}\left(\varphi^{\prime}, \zeta\right)\right]+\sum_{\substack{Z \in P_{1} \\
|Z|_{1} \geq 2}}^{Z=Y^{\prime}} \mathbb{E}_{j}\left[P_{j}^{Z}\left(\varphi^{\prime}, \zeta\right)\right]
\end{aligned}
$$

Then, Lemma 3.5 is a direct consequence of the following result.

Lemma 6.3 There exists $C(A, L, \alpha)>1$ such that, for $\varepsilon_{0}$ small enough, any $\mathcal{K}_{j}$ and $\dot{\mathcal{K}}_{j}$ with $\left|\mathcal{K}_{j}\right|_{\varepsilon_{0}, j},\left|\dot{\mathcal{K}}_{j}\right|_{\varepsilon_{0}, j} \leq 1$ and $Y^{\prime} \in \mathcal{P}_{j+1}^{c}$ : if $j=0$,

$$
\left\|\mathcal{R}_{0}-\dot{\mathcal{R}}_{0}\right\|_{h, T_{0}} \leq C(A, L, \alpha) \varepsilon_{0}[|s-\dot{s}|+|z-\dot{z}|]
$$

while, if $j=1, \ldots, R-1$,

$$
\left\|\mathcal{R}_{j}^{(m)}-\dot{\mathcal{R}}_{j}^{(m)}\right\|_{h, T_{j}} \leq C(A, L, \alpha) \varepsilon_{0}^{3}\left|\mathcal{K}_{j}-\dot{\mathcal{K}}_{j}\right|_{\varepsilon_{0}, j}
$$

for any $m=1,2, \ldots, 8$.

Remark. Many details of the construction of $K_{j+1}$ out of $K_{j}$ and $U_{j}$, (4.33), are designed with the goal of having (6.52), (6.53). In particular, the preliminary reblocking of $K_{j}$, with support on $j$-polymers, into $\widetilde{K}_{j}$, with support on $j+1$-polymers - a step not done in [5] - seems necessary here to obtain (6.55) form (6.54) through the new integration property (5.16).

Proof. First consider $\mathcal{R}_{j}^{(4)}$ that is the most instructive term. By Lemma 6.2, its bound is made of three kind of factors: a product of field regulators; a product of $A^{-1}$ 's; a product of $\left\|\mathcal{K}_{j}\right\|_{h, T_{j}}$ 's and $C(A, L, \alpha)$ 's. The field regulators stemming from the $P_{j}$ and $R_{j}$ factors can be merged together by Lemma 5.1; given disjoint $Z, X_{1} \in \mathcal{P}_{j+1}$,

$$
\begin{aligned}
& \prod_{D \in \mathcal{B}_{j+1}(Z)}\left[G_{j}^{\mathrm{str}}(\varphi, D)+G_{j+1}^{\mathrm{str}}\left(\varphi^{\prime}, D\right)\right] \prod_{Y \in \mathcal{C}_{j+1}\left(X_{1}\right)}\left[G_{j}(\varphi, Y)+G_{j+1}^{\mathrm{str}}\left(\varphi^{\prime}, Y\right)\right] \\
\leq & \sum_{\substack{W_{1} \in \mathcal{P}_{j+1}(Z) \\
W_{2} \in\left(\left(X_{1}\right)\right)_{j+1}}} G_{j+1}^{\mathrm{str}}\left(\varphi^{\prime}, Z \backslash W_{1}\right) G_{j+1}^{\mathrm{str}}\left(\varphi^{\prime}, X_{1} \backslash W_{2}\right) G_{j}\left(\varphi, W_{1} \cup W_{2}\right)
\end{aligned}
$$

where $\left(\left(X_{1}\right)\right)_{j+1}$ is the collections of subsets of $X_{1}$ made of unions of connected parts of $X_{1}$. There are no more than $2^{\left|\mathcal{C}_{j+1}\left(X_{1}\right)\right|+|Z|_{j+1}}$ terms in the sum above. Then take 
the expectation $\mathbb{E}_{j}$ in (6.54) using (5.16), and merge also the (strong) field regulators stemming from the bounds for the $J_{j}$ 's factors, to obtain the upper bound for (6.54)

$$
2^{\left|\mathcal{C}_{j+1}\left(X_{1}\right)\right|+|Z|_{j+1}} 2^{L^{2}\left|X_{1} \cup Z\right|_{j+1}} G_{j+1}\left(\varphi^{\prime}, \cup_{Y} D_{Y} \cup X_{1} \cup Z\right)
$$

Next, collect all the $A^{-1}$ factors from the bounds of $P_{j}$ 's, $R_{j}$ 's and $J_{j}$ 's to obtain $A^{-(1+\eta)\left|Y^{\prime}\right|_{j+1}}$. Finally, since the variation in the vector $\mathcal{K}_{j}$ can occur in each of the factors, that are no more than $\left|Y^{\prime}\right|_{j+1}$, we obtain:

$$
\begin{aligned}
& \left\|\mathcal{R}_{j}^{(4)}\left(\varphi^{\prime}, Y^{\prime}\right)-\dot{\mathcal{R}}_{j}^{(4)}\left(\varphi^{\prime}, Y^{\prime}\right)\right\|_{h, T_{j}\left(\varphi^{\prime}, Y^{\prime}\right)} \\
& \leq G_{j+1}\left(\varphi^{\prime}, Y^{\prime}\right) A^{-(1+\eta)\left|Y^{\prime}\right|_{j+1}}\left|\mathcal{K}_{j}-\dot{\mathcal{K}}_{j}\right|_{\varepsilon_{0}, j}\left|Y^{\prime}\right|_{j+1} 2^{L^{2}\left|Y^{\prime}\right|_{j+1}} \\
& \sum_{\substack{\mathcal{C}_{j+1}\left(X_{0} \cup X_{1}\right) \geq 1 \\
|Z|_{j+1}+\mathcal{C}_{j+1}\left(X_{0} \cup X_{1}\right) \geq 2}}^{\rightarrow Y^{\prime}}\left[2 C(A, L, \alpha) \varepsilon_{0}\right]^{|Z|_{j+1}+2\left|\mathcal{C}_{j+1}\left(X_{0} \cup X_{1}\right)\right|} .
\end{aligned}
$$

Notice the following facts: the number of term in the sum is not bigger than

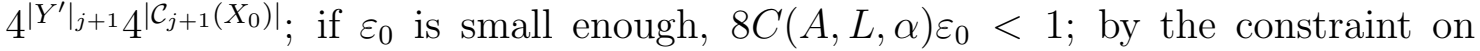
the sum there is a least factor $\left[8 C(A, L, \alpha) \varepsilon_{0}\right]^{3}$ in each summand; finally, for $A$ large enough, $\left(A^{-\eta} 2^{\left(L^{2}+2\right)}\right)^{\left|Y^{\prime}\right|_{j+1}}\left|Y^{\prime}\right|_{j+1}<1$. Therefore, for $C^{\prime}(A, L, A)>[8 C(A, L, \alpha)]^{3}$, an upper bound for (6.56) is

$$
C^{\prime}(A, L, \alpha) \varepsilon_{0}^{3}\left|\mathcal{K}_{j}-\dot{\mathcal{K}}_{j}\right|_{\varepsilon_{0}, j} G_{j+1}\left(\varphi^{\prime}, Y^{\prime}\right) A^{-\left|Y^{\prime}\right|_{j+1}}
$$

This proves $(\underline{6.53})$ for $m=4$. Now consider $\mathcal{R}_{j}^{(5)}$. It can be recasted as

$$
\begin{gathered}
\sum_{\mathcal{C}_{j+1}\left(X_{0} \cup X_{1}\right) \geq 1}^{\rightarrow Y^{\prime}} \sum_{\substack{W_{0} \in \mathcal{P}_{j}(W) \\
W_{1} \in \mathcal{P}_{j}\left(Y^{\prime} \backslash W\right)}}^{\left|W_{0} \cup W_{1}\right|_{j+1} \geq 1}\left(e^{-\mathcal{E}_{j} L^{2 j+2}}-1\right)^{\left|W_{0}\right|_{j+1}} J_{j}^{X_{0},(D)}\left(\varphi^{\prime}\right) \cdot \\
\cdot \mathbb{E}_{j}\left[P_{j}^{Z}\left(\varphi^{\prime}, \zeta\right) R_{j}^{X_{1}}\left(\varphi^{\prime}, \zeta\right)\right] \prod_{D_{1} \in \mathcal{B}_{j+1}\left(W_{1}\right)}\left(e^{U_{j+1}\left(\varphi^{\prime}, D_{1}\right)}-1\right) .
\end{gathered}
$$

Bound (6.53) for $m=5$ follows, mostly, as for $m=4$; so we just stress one detail: after field regulators are multiplied and integrated, we have to merge strong field regulators originated by the bound on (possibly, the variation of) $J_{j}$ 's, but also $e^{-U_{j+1}\left(\varphi^{\prime}, D_{1}\right)}-1$ 's; still, by construction, the final bound for the field regulators is $G_{j+1}\left(\varphi^{\prime}, Y^{\prime}\right)$, as wanted.

Bounds (6.53) for $m=7,8$ can be obtained in the very same fashion as (6.32); while cases $m=1,2,3,6$ are even simpler than the previous ones. This completes the proof of Lemma 3.5 . 


\section{Stable Manifold Theorem}

In this discussion, $\alpha^{2}=8 \pi$. Then, a natural way to recast (3.14) is

$$
\begin{aligned}
& s_{j+1}=s_{j}-a z_{j}^{2}+\mathcal{F}_{j}\left(s_{j}, z_{j}, K_{j}\right) \\
& z_{j+1}=z_{j}-b s_{j} z_{j}+\mathcal{M}_{j}\left(s_{j}, z_{j}, K_{j}\right) \\
& K_{j+1}=\mathcal{L}_{j}\left(K_{j}\right)+\mathcal{R}_{j}\left(z_{j}, s_{j}, K_{j}\right)
\end{aligned}
$$

for $a \equiv a(L)$ and $b \equiv b(L)$ given in Lemma 3.1, and

$$
\begin{aligned}
\mathcal{F}_{j}\left(s_{j}, z_{j}, K_{j}\right):=\mathcal{F}_{j}\left(K_{j}\right)-\left(a_{j}-a\right) z_{j}^{2} & \\
\mathcal{M}_{j}\left(s_{j}, z_{j}, K_{j}\right):= & L^{2} e^{-\frac{\alpha^{2}}{2} \Gamma_{j}(0)} \mathcal{M}_{j}\left(K_{j}\right)+\left[L^{2} e^{-\frac{\alpha^{2}}{2} \Gamma_{j}(0)}-1\right] z_{j} \\
& -\left[L^{2} e^{-\frac{\alpha^{2}}{2} \Gamma_{j}(0)} b_{j}-b\right] s_{j} z_{j} .
\end{aligned}
$$

The flow equation for $j=0$ is simpler and given in (3.13). We want to redefine the dynamical system so that it begins at step 1 instead of step 0 (and so to absorb the constants $a$ and $b)$. To this purpose, define $\left(x_{j}, y_{j}, W_{j}\right)_{j \geq 1}$ such that: $\left(x_{1}, y_{1}, W_{1}\right):=\left(b s_{1}, \sqrt{a b} z_{1}, 0\right)$, while, for $j \geq 2,\left(x_{j}, y_{j}, W_{j}\right):=\left(b s_{j}, \sqrt{a b} z_{j}, K_{j}\right)$. Accordingly the flow equation becomes

$$
\begin{aligned}
& x_{j+1}-x_{j}=-y_{j}^{2}+\widetilde{\mathcal{F}}_{j}\left(x_{j}, y_{j}, W_{j}\right) \\
& y_{j+1}-y_{j}=-x_{j} y_{j}+\widetilde{\mathcal{M}}_{j}\left(x_{j}, y_{j}, W_{j}\right) \\
& W_{j+1}=\mathcal{L}_{j}\left(W_{j}\right)+\widetilde{\mathcal{R}}_{j}\left(x_{j}, y_{j}, W_{j}\right) .
\end{aligned}
$$

with, for $j=1$ and $\lambda=\left[L^{2} e^{-\frac{\alpha^{2}}{2} \Gamma_{0}(0)}\right]^{-1}$,

$$
\begin{aligned}
& \widetilde{\mathcal{F}}_{1}\left(x_{1}, y_{1}, W_{1}\right):=b \mathcal{F}_{1}\left(s_{1}, z_{1}, \mathcal{R}_{0}\left(s_{1}, \lambda z_{1}\right)\right) \\
& \widetilde{\mathcal{M}}_{j}\left(x_{1}, y_{1}, W_{1}\right):=\sqrt{a b} \mathcal{M}_{1}\left(s_{1}, z_{1}, \mathcal{R}_{0}\left(s_{1}, \lambda z_{1}\right)\right) \\
& \widetilde{\mathcal{R}}_{1}\left(x_{1}, y_{1}, W_{1}\right):=\mathcal{L}_{1}\left(\mathcal{R}_{0}\left(s_{1}, \lambda z_{1}\right)\right)+\mathcal{R}_{1}\left(s_{1}, z_{1}, \mathcal{R}_{0}\left(s_{1}, \lambda z_{1}\right)\right) ;
\end{aligned}
$$

while, for $j \geq 2$,

$$
\begin{aligned}
& \widetilde{\mathcal{R}}_{j}\left(x_{j}, y_{j}, W_{j}\right):=\mathcal{R}_{j}\left(s_{j}, z_{j}, K_{j}\right), \\
& \widetilde{\mathcal{F}}_{j}\left(x_{j}, y_{j}, W_{j}\right):=b \mathcal{F}_{j}\left(s_{j}, z_{j}, K_{j}\right), \\
& \widetilde{\mathcal{M}}_{j}\left(x_{j}, y_{j}, W_{j}\right):=\sqrt{a b} \mathcal{M}_{j}\left(s_{j}, z_{j}, K_{j}\right) .
\end{aligned}
$$

Then, equations (3.13) and (3.14) for the RG flow are equivalent to (7.3). Note the following consequences of Lemmas 3.1, 3.3, 3.4 and 3.5. if $\left|x_{j}\right|,\left|y_{j}\right|,\left|\dot{x}_{j}\right|,\left|\dot{y}_{j}\right| \leq \varepsilon_{j}$ 
and $\left\|W_{j}\right\|_{h, j},\left\|\dot{W}_{j}\right\|_{h, j} \leq \varepsilon_{j}^{2}$

$$
\begin{gathered}
\left|\widetilde{\mathcal{F}}_{j}\left(x_{j}, y_{j}, W_{j}\right)-\widetilde{\mathcal{F}}_{j}\left(\dot{x}_{j}, \dot{y}_{j}, \dot{W}_{j}\right)\right| \leq C(L) L^{-\frac{j}{4}}\left|y_{j}^{2}-\dot{y}_{j}^{2}\right| \\
+C(L) A^{-1}\left[\left\|W_{j}-\dot{W}_{j}\right\|_{h, T_{j}}+\delta_{j, 1} \varepsilon_{1}\left(\left|x_{1}-\dot{x}_{1}\right|+\left|y_{1}-\dot{y}_{1}\right|\right)\right] \\
\left|\widetilde{\mathcal{M}}_{j}\left(x_{j}, y_{j}, W_{j}\right)-\widetilde{\mathcal{M}}_{j}\left(\dot{x}_{j}, \dot{y}_{j}, \dot{W}_{j}\right)\right| \leq c L^{-\frac{j}{4}}\left|y_{j}-\dot{y}_{j}\right|+C(L) L^{-\frac{j}{4}}\left|x_{j} y_{j}-\dot{x}_{j} \dot{y}_{j}\right| \\
+C(L) A^{-1}\left[\left\|W_{j}-\dot{W}_{j}\right\|_{h, T_{j}}+\delta_{j, 1} \varepsilon_{1}\left(\left|x_{1}-\dot{x}_{1}\right|+\left|y_{1}-\dot{y}_{1}\right|\right)\right] \\
\left|\widetilde{\mathcal{R}}_{j}\left(x_{j}, y_{j}, W_{j}\right)-\widetilde{\mathcal{R}}_{j}\left(\dot{x}_{j}, \dot{y}_{j}, \dot{W}_{j}\right)\right| \leq c\left(L^{-\vartheta}+A^{-\eta}\right) \delta_{j, 1} \varepsilon_{1}\left(\left|x_{1}-\dot{x}_{1}\right|+\left|y_{1}-\dot{y}_{1}\right|\right) \\
\quad+C(A, L)\left[\varepsilon_{j}^{2}\left|x_{j}-\dot{x}_{j}\right|+\varepsilon_{j}^{2}\left|y_{j}-\dot{y}_{j}\right|+\varepsilon_{j}\left\|W_{j}-\dot{W}_{j}\right\|_{h, T_{j}}\right]
\end{gathered}
$$

The RG equations that we are describing here are well defined, and so considered, in "infinite volume", i.e. for any $j \geq 1$ and with polymer activities defined on $\mathbb{Z}^{2}$. Theorem 3.6 (which is about the RG on finite lattice $\Lambda$ and with $j=1, \ldots, R$ ) will be a corollary of the following result - see the remark after the proof. Hypotheses slightly more general than what we need, since $W_{1}$ is not assumed to be 0 .

Theorem 7.1 Consider the solution of 7.3) with initial data $\left(x_{1}, y_{1}, W_{1}\right)$. If $L$ and $A$ are large enough, if $\left|y_{1}\right| \leq \varepsilon_{1}$ for a small $\varepsilon_{1} \equiv \varepsilon_{1}(A, L)$ and if $\left\|W_{1}\right\|_{h, T_{1}}$ is small enough w.r.t $\left|y_{1}\right|$, there exist $x_{1}=\Sigma\left(y_{1}\right)$ such that $\left|x_{1}\right| \leq 2 \varepsilon_{1}$ and

$$
x_{j}=\left|q_{j}\right|+O\left(j^{-\frac{3}{2}}\right), \quad y_{j}=q_{j}+O\left(j^{-\frac{3}{2}}\right), \quad\left\|W_{j}\right\|_{h, T_{j}}=O\left(j^{-3}\right),
$$

where $q_{j}$ is the same defined in (3.19) with $q_{1}:=y_{1}$.

Proof. Without loss of generality, assume $y_{1}$ positive. The strategy - partially inspired to [10] - is to mimic the treatment of the continuous version of (7.3). We look for a solution $\left(x_{j}, y_{j}, W_{j}\right)$ of (7.3) into the form $x_{j}=q_{j}+u_{j}, y_{j}=q_{j}+v_{j}$, where $u_{j}$, $v_{j}, W_{j}$ are unknowns with initial data $u_{1}=x_{1}-y_{1}, v_{1}=0$ and $W_{1}$. By the identity $q_{j+1}-q_{j}=-q_{j} q_{j+1}$, the equations for $u_{j}$ and $v_{j}$ are

$$
\begin{aligned}
& u_{j+1}-u_{j}=-2 v_{j} q_{j}+\mathcal{U}_{j}\left(u_{j}, v_{j}, W_{j}\right) \\
& v_{j+1}-v_{j}=-u_{j} q_{j}-v_{j} q_{j}+\mathcal{V}_{j}\left(u_{j}, v_{j}, W_{j}\right)
\end{aligned}
$$

for $\mathcal{U}_{j}\left(u_{j}, v_{j}, W_{j}\right):=-v_{j}^{2}-q_{j}^{2} q_{j+1}+\widetilde{\mathcal{F}}_{j}\left(x_{j}, y_{j}, W_{j}\right)$ and $\mathcal{V}_{j}\left(u_{j}, v_{j}, W_{j}\right):=-u_{j} v_{j}-$ $q_{j}^{2} q_{j+1}+\widetilde{\mathcal{M}}_{j}\left(x_{j}, y_{j}, W_{j}\right)$. In order to diagonalize the linear part, introduce the stable direction, $w_{j}^{+}:=u_{j}+2 v_{j}$, and the unstable direction, $w_{j}^{-}:=u_{j}-v_{j}$, that have initial data $w_{1}^{+}=w_{1}^{-}=x_{1}-y_{1}$ and satisfy

$$
\begin{aligned}
& w_{j+1}^{+}-w_{j}^{+}=\left(-2 q_{j+1}+q_{j+1}^{2}\right) w_{j}^{+}+\mathcal{W}_{j}^{+}\left(u_{j}, v_{j}, W_{j}\right) \\
& w_{j+1}^{-}-w_{j}^{-}=q_{j} w_{j}^{-}+\mathcal{W}_{j}^{-}\left(u_{j}, v_{j}, W_{j}\right)
\end{aligned}
$$


for $\mathcal{W}_{j}^{+}:=\mathcal{U}_{j}+2 \mathcal{V}_{j}+\left[2 q_{j}-q_{j+1}\right] q_{j+1} w_{j}^{+}$and $\mathcal{W}_{j}^{-}:=\mathcal{U}_{j}-\mathcal{V}_{j}$ (changing the linear order of the former equation into $\left(-2 q_{j+1}+q_{j+1}^{2}\right) w_{j}^{+}$, up to a correction $O\left(q_{j}^{2}\right) w_{j}^{+}$ that is absorbed into $\mathcal{W}_{j}^{+}$, will make easier the next step). Since $q_{j}$ is strictly positive, $w_{j}^{+}$is driven to zero by the linear term in any case; whereas $w_{j}^{-}$converges to zero only for a special initial $w_{1}^{+}=w_{1}^{-}=x_{1}-y_{1}$, to be found. With some simple algebra, turn (7.11), together to the condition $w_{\infty}^{-}=0$, into a form that resembles the integral equation for the continuous flow:

$$
\begin{aligned}
& w_{j}^{+}=\left(\frac{q_{j}}{q_{1}}\right)^{2} w_{1}^{+}+\sum_{s=1}^{j-1}\left(\frac{q_{j}}{q_{s+1}}\right)^{2} \mathcal{W}_{s}^{+}\left(u_{j}, v_{j}, W_{j}\right) \\
& w_{j}^{-}=-\sum_{s \geq j} \frac{q_{s+1}}{q_{j}} \mathcal{W}_{s}^{-}\left(u_{j}, v_{j}, W_{j}\right) \\
& W_{j}=\mathcal{L}_{j-1,1} W_{1}+\sum_{s=1}^{j-1} \mathcal{L}_{j-1, s+1} \mathcal{W}_{s}^{0}\left(u_{j}, v_{j}, W_{j}\right)
\end{aligned}
$$

for $\mathcal{L}_{n, m}:=\mathcal{L}_{n} \circ \mathcal{L}_{n-1} \circ \cdots \circ \mathcal{L}_{m}$ and $\mathcal{W}_{j}^{0}\left(u_{j}, v_{j}, W_{j}\right):=\widetilde{\mathcal{R}}_{j}\left(x_{j}, y_{j}, K_{j}\right)$. (As in standard conventions, when a counter ranges over an empty set, the corresponding summation is zero, the corresponding chain of convolutions is the identity.)

Introduce a vector notation, $\underline{w}=\left(w_{j}^{+}, w_{j}^{-}, W_{j}\right)_{j \geq 1}$, and the norm

$$
\|\underline{w}\|=\sup _{j \geq 1} \max \left\{\left(\tau h_{j}\right)^{-1}\left|w_{j}^{+}\right|, 2\left(\tau h_{j}\right)^{-1}\left|w_{j}^{-}\right|,\left(\tau h_{j}\right)^{-2}\left\|W_{j}\right\|_{h, T_{j}}\right\}
$$

where $h_{j}$ is the sequence $h_{j}=y_{1}\left[1+y_{1}(j-1)\right]^{-\frac{3}{2}}$ and $\tau>0$. It is easy to see that $\mathbb{W}=\{\underline{w}:\|\underline{w}\| \leq 1\}$ is a Banach space. Then define the operator $\mathcal{T}=$ $\left(\mathcal{T}_{j}^{+}, \mathcal{T}_{j}^{-}, \mathcal{T}_{j}^{0}\right)_{j}$ such that $(7.12)$ reads $\underline{w}=\mathcal{T} \underline{w}$, i.e.

$$
w_{j}^{+}=\mathcal{T}_{j}^{+}(\underline{w}), \quad w_{j}^{-}=\mathcal{T}_{j}^{-}(\underline{w}), \quad W_{j}=\mathcal{T}_{j}^{0}(\underline{w}) .
$$

We shall prove that, if $\tau$ is small enough (independently of $A$ and $L$ ), $\mathcal{T}$ is a contraction of $\mathbb{W}$; then $\underline{w}=\mathcal{T} \underline{w}$ has a unique solution in $\mathbb{W}$.

Define $p_{j}:=2^{-(j-1)}$ and the operators

$$
T_{j}^{+}(\mathcal{W}):=\sum_{s=1}^{j-1}\left(\frac{q_{j}}{q_{s+1}}\right)^{2} \mathcal{W}_{s}, \quad T_{j}^{-}(\mathcal{W}):=-\sum_{s \geq j} \frac{q_{s+1}}{q_{j}} \mathcal{W}_{s}
$$

For $\alpha= \pm$,

$$
\left|T_{j}^{\alpha}\left(h^{2}\right)\right| \leq C h_{j}, \quad\left|T_{j}^{\alpha}(p q)\right| \leq C h_{j} .
$$

By definition of $\mathcal{W}_{j}^{\alpha}$, for $\alpha= \pm$, if $L$ is large enough,

$$
\left|\mathcal{W}_{j}^{\alpha}\left(u_{j}, v_{j}, W_{j}\right)\right| \leq C h_{j}^{2}\left[\tau^{2}+q_{1}\right]+C(L)\left[p_{j} q_{j}^{2}+A^{-1} h_{j}^{2}\right]+C L^{-\frac{1}{4}} p_{j} q_{j}
$$


therefore, using (7.14),

$$
\left|T_{j}^{\alpha}\left(\mathcal{W}^{\alpha}\right)\right| \leq C h_{j}\left[\tau^{2}+q_{1}+L^{-\frac{1}{4}}+C(L)\left(A^{-1}+q_{1}\right)\right] .
$$

Now consider two vectors in $\mathbb{W}, \underline{w}=\left(u_{j}, v_{j}, W_{j}\right)_{j}$ and $\underline{\dot{w}}=\left(\dot{u}_{j}, \dot{v}_{j}, \dot{W}_{j}\right)_{j}$; then

$$
\begin{aligned}
& \left|\mathcal{W}_{j}^{\alpha}\left(u_{j}, v_{j}, W_{j}\right)-\mathcal{W}_{j}^{\alpha}\left(\dot{u}_{j}, \dot{v}_{j}, \dot{W}_{j}\right)\right| \\
& \leq C\|\underline{w}-\underline{\dot{w}}\| \tau\left[h_{j}^{2}\left[\tau+q_{1}\right]+C(L) A^{-1} h_{j}^{2}+L^{-\frac{1}{4}} p_{j} q_{j}\right]
\end{aligned}
$$

hence, by (7.14),

$$
\left|T_{j}^{\alpha}\left(\mathcal{W}^{\alpha}\right)-T_{j}^{\alpha}\left(\dot{\mathcal{W}}^{\alpha}\right)\right| \leq C\|\underline{w}-\underline{\dot{w}}\| \tau h_{j}\left[\tau+q_{1}+L^{-\frac{1}{4}}+C(L) A^{-1}\right] .
$$

Finally, from (7.16) and (7.18), assuming $\tau$ small enough so that $16 C \tau \leq 1, L$ large enough so that $16 C L^{-\frac{1}{4}} \leq \tau, A$ large enough so that $16 C(L) A^{-1} C \leq \tau$ and finally $q_{1}=\left|y_{1}\right|$ small enough so that $16 C(L) q_{1} C \leq \tau$,

$$
\left|T_{j}^{\alpha}\left(\mathcal{W}^{\alpha}\right)\right| \leq \frac{\tau h_{j}}{2} \quad\left|T_{j}^{\alpha}\left(\mathcal{W}^{\alpha}\right)-T_{j}^{\alpha}\left(\dot{\mathcal{W}}^{\alpha}\right)\right| \leq\|\underline{w}-\underline{\dot{w}}\| \frac{\tau h_{j}}{4} .
$$

Now consider the third of (7.12). For a polymer activity $\mathcal{W}_{j}$, define

$$
T_{j}^{0}(\mathcal{W})=\sum_{s=1}^{j-1} \mathcal{L}_{j-1, s+1} \mathcal{W}_{s}
$$

Suppose that $\left\|\mathcal{W}_{j}\right\|_{h, T_{j}} \leq h_{j}^{2}$; if $\left(L^{-\vartheta}+A^{-\eta}\right)$ is small enough ( $c f r$. Lemma 3.4),

$$
\left\|\left(T_{0} Q\right)_{j}\right\|_{j} \leq C h_{j}^{2} .
$$

By (7.8)

$$
\left\|\mathcal{W}_{j}^{0}\left(u_{j}, v_{j}, W_{j}\right)\right\|_{h, T_{j+1}} \leq C\left[L^{-\vartheta}+A^{-\eta}+C(A, L) q_{1}\right] h_{j}^{2}
$$

and then

$$
\left\|T_{j}^{0}\left(\mathcal{W}^{0}\right)\right\|_{h, T_{j}} \leq C\left[L^{-\vartheta}+A^{-\eta}+C(A, L) q_{1}\right] h_{j}^{2}
$$

Besides, given two vectors in $\mathbb{W}, \underline{w}=\left(u_{j}, v_{j}, W_{j}\right)_{j}$ and $\underline{\dot{w}}=\left(\dot{u}_{j}, \dot{v}_{j}, \dot{W}_{j}\right)_{j}$,

$$
\begin{aligned}
\left\|\mathcal{W}_{j}^{0}\left(u_{j}, v_{j}, W_{j}\right)-\mathcal{W}_{j}^{0}\left(\dot{u}_{j}, \dot{v}_{j}, \dot{W}_{j}\right)\right\|_{h, T_{j+1}} \\
\leq \tau\|\underline{w}-\underline{\dot{w}}\| C\left[L^{-\vartheta}+A^{-\eta}+C(A, L) q_{1}\right] h_{j}^{2} .
\end{aligned}
$$

and using (7.20) again,

$$
\left\|T_{j}^{0}\left(\mathcal{W}^{0}\right)-T_{j}^{0}\left(\dot{\mathcal{W}}^{0}\right)\right\|_{h, T_{j}} \leq \tau\|\underline{w}-\underline{\dot{w}}\| C\left[L^{-\vartheta}+A^{-\eta}+C(A, L) q_{1}\right] h_{j}^{2} .
$$


For $L$ and $A$ large enough, $4 C\left[L^{-\vartheta}+A^{-\eta}\right] \leq \tau^{2}$; then, for $q_{1}$ small enough, $4 C(A, L, \alpha) q_{1} C<\tau^{2} ;$ then

$$
\left\|T_{j}^{0}\left(\mathcal{W}^{0}\right)\right\|_{h, T_{j}} \leq\left(\tau h_{j}\right)^{2}, \quad\left\|T_{j}^{0}\left(\mathcal{W}^{0}\right)-T_{j}^{0}\left(\dot{\mathcal{W}}^{0}\right)\right\|_{h, T_{j}} \leq \frac{\left(\tau h_{j}\right)^{2}}{2}\|\underline{w}-\underline{\dot{w}}\| .
$$

Finally, consider the operator $\mathcal{T}$. By (17.19) and (17.25), if $\underline{w} \in \mathbb{W}$,

$$
\begin{gathered}
\left|\mathcal{T}_{j}^{+}(\underline{w})\right| \leq\left(\frac{q_{j}}{q_{1}}\right)^{2}\left|w_{1}^{-}\right|+\left|T_{j}^{+}\left(\mathcal{W}^{+}\right)\right| \leq \tau h_{j}, \quad\left|\mathcal{T}_{j}^{-}(\underline{w})\right| \leq\left|T_{j}^{-}\left(\mathcal{W}^{-}\right)\right| \leq \frac{\tau h_{j}}{2} \\
\left\|\mathcal{T}_{j}^{0}(\underline{w})\right\|_{h, T_{j}} \leq\left\|T_{j}^{0}\left(\mathcal{W}^{0}\right)\right\|_{h, T_{j}} \leq\left(\tau h_{j}\right)^{2}
\end{gathered}
$$

and, if $\underline{w}, \underline{\dot{w}} \in \mathbb{W}$, with similar derivation

$$
\begin{gathered}
\left|\mathcal{T}_{j}^{+}(\underline{w})-\mathcal{T}_{j}^{+}(\underline{\dot{w}})\right| \leq \frac{\tau h_{j}}{2}\|\underline{w}-\underline{\dot{w}}\|, \quad\left|\mathcal{T}_{j}^{-}(\underline{w})-\mathcal{T}_{j}^{-}(\underline{\dot{w}})\right| \leq \frac{\tau h_{j}}{4}\|\underline{w}-\underline{\dot{w}}\|, \\
\left\|\mathcal{T}_{j}^{0}(\underline{w})-\mathcal{T}_{j}^{0}(\underline{\dot{w}})\right\|_{h, T_{j}} \leq \frac{\left(\tau h_{j}\right)^{2}}{2} .
\end{gathered}
$$

Hence $\mathcal{T}$ is a contraction on $\mathbb{W}$ with norm $1 / 2$. The Theorem is proven.

Remark. Using the same choice $\Sigma\left(y_{1}\right)$ for $x_{1}$ found above in the infinite-volume case, the very same bounds for the decay of $x_{j}, y_{j}, W_{j}$ are valid also for the finitevolume RG flow. In fact, the flows of $x_{j}$ and $y_{j}$ are unchanged, for $\widetilde{F}_{j}$ and $\widetilde{\mathcal{M}}_{j}$ are built out of $W_{j}$ 's living on small sets only. The flow of $W_{j}$, instead, is changed, but only when the support of $W_{j}$ is a polymer that wraps around the torus $\Lambda$ - a nonsmall at any scale $j=1,2, \ldots, R-1$ : since $W_{j}$ is anyways a stable (i.e. contracting) direction of the flow, this change does not require a different initial datum $\left(x_{1}, y_{1}\right)$. Therefore, Theorem 7.1 indirectly implies Theorem 3.6.

\section{A Functional Integral Formula}

\section{A.1 Siegert-Kac transformation}

We begin with stressing two properties of the lattice Yukawa potential in two dimensions, (2.3). First, the self-energy of a particle,

$$
W_{\Lambda}(0 ; m)=\frac{1}{|\Lambda|} \sum_{p \in \Lambda^{*}} \frac{1}{m^{2}-\widehat{\Delta}(p)}
$$

is positive divergent. Next, if we define the normalized interaction $W_{\Lambda}(x \mid 0 ; m):=$ $W_{\Lambda}(x ; m)-W_{\Lambda}(0 ; m)$, we find

$$
W_{\Lambda}(x \mid 0):=\lim _{m \rightarrow 0} W_{\Lambda}(x \mid 0 ; m)=\frac{1}{|\Lambda|} \sum_{p \in \Lambda^{*} \backslash\{0\}} \frac{e^{i p x}-1}{-\widehat{\Delta}(p)} ;
$$


then, in the infinite volume, $W(x \mid 0):=\lim _{\Lambda \rightarrow \infty} W_{\Lambda}(x \mid 0)$ has large $|x|$ behavior

$$
W(x \mid 0)=-\frac{1}{2 \pi} \ln |x|+O(1)
$$

which is what is called the Coulomb potential in two-dimensions. Now consider (2.4) written in terms of normalized interactions and self-energies,

$$
H_{\Lambda}(\omega ; m)=\frac{1}{2} \sum_{i, j=1}^{n} \sigma_{i} \sigma_{j} W_{\Lambda}\left(x_{i}-x_{j} \mid 0 ; m\right)+\frac{1}{2}\left(\sum_{i=1}^{n} \sigma_{i}\right)^{2} W_{\Lambda}(0 ; m) .
$$

As consequence of (A.2) and (A.3), in the limit $m \rightarrow 0$, the latter term of (A.4) assign an infinite energy, i.e. a zero probabilistic weight, to configurations of $\Omega_{n}$ that are not globally neutral. For neutral configurations, instead, the latter term is zero and the particle interacts through the normalized potential. This justifies the use of the Yukawa potential as regularization of the Coulomb one. Notice that $W_{\Lambda}(x ; m)$ is positive definite and thus $e^{-H_{\Lambda}(\omega ; m)} \leq 1$; then, in the formula for the partition function, the sum on $n$ can be taken before the limit $m \rightarrow 0$, for the resulting series is convergent uniformly in $m$ :

$$
Z_{\Lambda}(\beta)=\lim _{m \rightarrow 0} \sum_{n \geq 0} \frac{z^{n}}{n !} \sum_{\omega \in \Omega_{n}} e^{-\beta H_{\Lambda}(\omega ; m)} .
$$

Now we introduce the Siegert-Kac construction. Since $W_{\Lambda}(x ; m)$ is strictly-positive definite, introduce at any site $x \in \Lambda$ a random variable, or 'field', $\varphi_{x}$, with Gaussian measure $d P_{R, 0}(\varphi ; m)$ determined by

$$
\int d P_{R, 0}(\varphi ; m) \varphi_{x}=0, \quad \int d P_{R, 0}(\varphi ; m) \varphi_{x} \varphi_{y}=W_{\Lambda}(x-y ; m) .
$$

Accordingly, Boltzmann weights of a given configuration in $\Omega_{n}$ can be re-written as characteristic functions of the Gaussian measure

$$
\int d P_{R, 0}\left(\frac{\varphi}{\sqrt{\beta}} ; m\right) \exp \left(i \sum_{j=1}^{n} \sigma_{j} \varphi_{x_{j}}\right)=e^{-\beta H_{\Lambda}(\omega ; m)} .
$$

Notice that at this point of the analysis the Gaussian measure is finite-dimensional and non-degenerate; then its density has an explicit formula. For RG analysis purposes, we want to consider a part of the Gaussian density as an integrand: for any $s \in[0,1 / 2]$, if $\alpha^{2}:=\beta(1-s)$ and replacing $m$ with $\frac{m}{\sqrt{1-s}}$,

$$
d P_{R, 0}\left(\frac{\varphi}{\sqrt{\beta}} ; \frac{m}{\sqrt{1-s}}\right)=d P_{R, 0}\left(\frac{\varphi}{\alpha} ; m\right) \exp \left\{\frac{s}{2 \alpha^{2}} \sum_{\substack{x \in \Lambda \\ \mu \in \widehat{e}}}\left(\partial^{\mu} \varphi_{x}\right)^{2}\right\} \mathcal{N}_{\Lambda}^{1 / 2}(s ; m)
$$


where $\mathcal{N}_{\Lambda}$ takes into account the different normalization of the two measures

$$
\mathcal{N}_{\Lambda}(s ; m)=\prod_{k \in \Lambda^{*}} \frac{m^{2}-(1-s) \widehat{\Delta}(k)}{m^{2}-\widehat{\Delta}(k)} .
$$

With some algebra, the functional integral representation of the partition function of the Coulomb Gas is

$$
Z_{\Lambda}(\beta)=\lim _{m \rightarrow 0} \int d P_{R, 0}(\varphi ; m) e^{\mathcal{V}(\varphi)}
$$

for $\mathcal{V}$ given by (3.5). To obtain (3.1) we need a decomposition of the fields.

\section{A.2 Multiscale decomposition.}

Suppose there exist positive-definite functions $\Gamma_{0}(x ; m), \Gamma_{1}(x ; m), \ldots, \Gamma_{R-1}(x ; m)$ and $\Gamma_{\geq R}(x ; m)$ such that

$$
W_{\Lambda}(x ; m)=\sum_{j=0}^{R-1} \Gamma_{j}(x ; m)+\Gamma_{\geq R}(x ; m) .
$$

By standard theory of (finite dimensional) Gaussian processes, $\varphi$ can be decomposed into $R+1$ random variables,

$$
\varphi_{x}=\zeta_{x}^{(R)}+\zeta_{x}^{(R-1)}+\cdots+\zeta_{x}^{(0)}
$$

that are independent and Gaussian:

$$
d P_{R, 0}(\varphi ; m)=d P_{\geq R}\left(\zeta^{(R)} ; m\right) d P_{R-1}\left(\zeta^{(R-1)} ; m\right) \cdots d P_{0}\left(\zeta^{(R-1)} ; m\right)
$$

for $d P_{j}$ and $d P_{\geq R}$ Gaussian measures with covariances $\Gamma_{j}$ and $\Gamma_{\geq R}$, respectively.

Decomposition (A.9) can be obtained in many ways. Here we take advantage of a similar decomposition for $\bar{W}(x ; m)$, the massive covariance for the infinite lattice, $\mathbb{Z}^{2}$, derived in [9], but implemented on a finer set of scales ( $c f r$. Sec. D). Suppose $L=\gamma^{M}$, for $\gamma$ and $M$ positive integers; $\gamma$ odd. The decomposition of the covariance that we shall consider is done on scales $\gamma^{h}$, for integer $h>0$. Define, for $j=0, \ldots, R-1$,

$$
I_{j, j+1}:=\left\{j+m \log _{L} \gamma \mid m=0,1 \ldots, M-1\right\} ;
$$

also, define $Q_{h}:=\left[-\pi \gamma^{h}, \pi \gamma^{h}\right]$ and $Q:=Q_{0}$.

Theorem A.1 There exists a positive-definite $\bar{\Gamma}_{\geq R}(x ; m)$ and, for any $j=0,1, \ldots, R$ 1 and any $s \in I_{j, j+1}$, a positive-definite $C_{s}(x ; m)$ such that

$$
\bar{W}(x ; m)=\sum_{j=0}^{R-1} \bar{\Gamma}_{j}(x ; m)+\bar{\Gamma}_{\geq R}(x ; m), \quad \bar{\Gamma}_{j}(x ; m)=\sum_{s \in I_{j, j+1}} C_{s}(x ; m),
$$


with $C_{s}(x ; m)=0$ if $|x| \equiv \max \left\{x_{0}, x_{1}\right\}>\gamma^{s M+1} / 2$. Besides, for any $h / M \in I_{j, j+1}$, there exists $F_{h}(p ; m)$, defined for $p \in Q_{h}$, such that, if $h=s M$,

$$
\begin{aligned}
& C_{s}(x ; m)=\int_{Q} \frac{d^{2} p}{(2 \pi)^{2}} \frac{e^{i p x}}{m^{2}-\widehat{\Delta}(p)}\left[F_{h}\left(\gamma^{h} p ; m\right)-F_{h+1}\left(\gamma^{h+1} p ; m\right)\right], \\
& \bar{\Gamma}_{\geq R}(x ; m)=\int_{Q} \frac{d^{2} p}{(2 \pi)^{2}} \frac{e^{i p x}}{m^{2}-\widehat{\Delta}(p)} F_{M R}\left(\gamma^{M R} p ; m\right) .
\end{aligned}
$$

The proof (without the finer scales, i.e. for $\gamma$ replaced by $12 L$ ) is in $[9$. Notice that, as opposed to the full interactions $W_{\Lambda}(x ; m)$ or $\bar{W}(x ; m)$ that are strictly positivedefinite, the $C_{s}$ 's might be positive-definite only, i.e. the corresponding Gaussian measure might be degenerate. Anyways notice that, for example, if $C_{s}=g_{s} \circ g_{s}$ for positive-definite $g_{s}$, and if $\mathbb{E}_{C}$ is the expectation w.r.t. the Gaussian measure with covariance $C(x-y)$, then, for any integrable function $F$,

$$
\mathbb{E}_{C_{s}}[F(z)]=\mathbb{E}_{I}\left[F\left(g_{s} \circ z\right)\right]
$$

where $I(x-y):=\delta_{x, y}$ and hence strictly positive-definite (see App. of [13]).

There is a standard way to construct the periodic $W_{\Lambda}(x ; m)$ out of its infinite volume version $\bar{W}(x ; m)$,

$$
W_{\Lambda}(x ; m)=\sum_{y \in \mathbb{Z}^{2}} \bar{W}\left(x+L^{R} y ; m\right) .
$$

By finite support of $C_{s}, \bar{\Gamma}_{j}(x ; m)=0$ if $|x|>L^{j+1} / 2$; therefore by simple arguments (A.12), through (A.15), implies (A.9) for

$$
\Gamma_{j}(x ; m)=\bar{\Gamma}_{j}(x ; m), \quad \Gamma_{\geq R}(x ; m)=\frac{1}{|\Lambda|} \sum_{p \in \Lambda^{*}} \frac{e^{i p x}}{m^{2}-\widehat{\Delta}(p)} F_{M R}\left(L^{R} p ; m\right) .
$$

[9] provides also some properties of $F_{h}(p ; m)$ 's that we shall need below.

Lemma A.2 Given an integer $h \geq 0$, for $n=1,2, \ldots, h$, there are complex functions $\widehat{A}_{h, n}(p, m)$, defined and differentiable for $p \in Q_{h}$ and $m$ in a neighborhood of 0 , such that

$$
F_{0}(p ; m)=1, \quad F_{h}(p ; m)=\prod_{n=0}^{h-1}\left|\widehat{A}_{h, n}(p, m)\right|^{2} .
$$

Besides, there exists a constant $c \equiv c(\gamma)$ such that

$$
\begin{gathered}
\left|\widehat{A}_{h, n}(p ; m)\right| \leq 1, \\
\widehat{A}_{h, n}(0 ; 0)=1, \quad\left|1-\widehat{A}_{h, n}(p ; 0)\right| \leq c|p| \gamma^{-(h-n)},
\end{gathered}
$$




$$
\begin{gathered}
\left|\widehat{A}_{h, n}(p ; m)\right| \leq \frac{c}{1+\left(\gamma^{-(h-n-1)} p\right)^{4}}, \\
\left|\widehat{A}_{h, n}(p ; 0)-\widehat{A}_{h+1, n+1}(p ; 0)\right| \leq 2 c \gamma^{-n} .
\end{gathered}
$$

W.r.t. 9], here we abridged the notation $\widehat{A}_{\varepsilon_{h}, h-n}^{m^{2}}\left(R_{h-n}\right)(p)$ into $\widehat{A}_{h, n}\left(p ; \gamma^{-h} m\right)$. Therefore the above estimates (A.17), (A.18), (A.19) (A.20) correspond to formula (3.12), formula (6.17), Lemma 5.4 and Lemma 6.7 of [9], respectively; to optimize the constants, read off that paper the case $n=1$ only; the other values of $n$ can be obtained by scaling (3.26) of [9].

A direct consequence of (A.17), (A.18) and (A.19) is that

$$
0 \leq 1-F_{h}(p ; 0)=c|p|, \quad\left|F_{h}(p ; m)\right| \leq \frac{c}{1+p^{4}} .
$$

Now we can complete the proof of (3.1). Define $d P_{R-1,0}(\varphi ; m)$ as the Gaussian measure corresponding to the covariance $\Gamma_{R-1,0}(x ; m)=\Gamma_{R-1}(x ; m)+\Gamma_{R-2}(x ; m)+$ $\cdots+\Gamma_{0}(x ; m)$, and neglect the $m$ dependence in measures when $m=0$, i.e.

$$
d P_{R-1,0}(\varphi):=d P_{R-1,0}(\varphi ; 0), \quad d P_{j}(\varphi):=d P_{j}(\varphi ; 0) .
$$

(3.1) is a consequence of the following result.

\section{Lemma A.3}

$$
\lim _{m \rightarrow 0} \int d P_{R, 0}(\varphi ; m) e^{\mathcal{V}(\varphi)}=\lim _{m \rightarrow 0} \int d P_{\geq R}(\zeta ; m) \int d P_{R-1,0}(\varphi) e^{\mathcal{V}(\zeta+\varphi)} .
$$

Proof. It is an explicit check. For masses $m$ and $m^{\prime}$, define the Yukawa potential

$$
T_{\Lambda}\left(x ; m, m^{\prime}\right):=\frac{1}{|\Lambda|} \sum_{p \in \Lambda^{*}} e^{i p x} \frac{(1-s)\left[\widehat{\Gamma}_{R-1,0}\left(p ; m^{\prime}\right)+\widehat{\Gamma}_{\geq R}(p ; m)\right]}{1+s\left[\widehat{\Gamma}_{R-1,0}\left(p ; m^{\prime}\right)+\widehat{\Gamma}_{\geq R}(p ; m)\right] \widehat{\Delta}(p)} .
$$

In particular, notice that $W_{\Lambda}\left(x ; \frac{m}{\sqrt{1-s}}\right)=T_{\Lambda}(x ; m, m)$. Consider $T_{\Lambda}(x ; m, 0)$ instead: it is well-defined and positive-definite; $T_{\Lambda}(0 ; m, 0)$ is positive divergent for $m \rightarrow 0$; and $T_{\Lambda}(x \mid 0):=\lim _{m \rightarrow 0}\left[T_{\Lambda}(x ; m, 0)-T_{\Lambda}(0 ; m, 0)\right]=W_{\Lambda}(x \mid 0)$. This means that there is no change in (A.5) if we replace $W_{\Lambda}\left(x ; \frac{m}{\sqrt{1-s}}\right)$ with $T_{\Lambda}(x ; m, 0)$. The r.h.s. member of (A.22) stems from repeating all the derivations of Section A.1 for the latter potential. 


\section{A.3 Explicit Computations}

In order to prove (3.4) and (3.15) we need explicit computations that involve the covariances. For $p \in \mathbb{R}^{2}$, consider the differentiable function $u(p)=\lim _{h \rightarrow \infty} F_{h}(p ; 0)$; and define the covariance

$$
\widetilde{C}(x):=\int \frac{d^{2} p}{(2 \pi)^{2}} e^{i x p} \frac{u(p)-u(\gamma p)}{p^{2}} .
$$

By construction, $u(0)=1$ and, uniformly in $\vartheta, \lim _{\rho \rightarrow \infty} u(\rho \cos \vartheta, \rho \sin \vartheta)=0$

Lemma A.4 For any $j=0,1, \ldots, R-1$ and $s \in I_{j, j+1}$, if $h=s M$,

$$
\left|C_{s}(x ; 0)-\widetilde{C}\left(\gamma^{-h} x\right)\right| \leq c \gamma^{-\frac{h}{4}}, \quad\left|\partial^{\mu} C_{s}(x ; 0)-\gamma^{-h} \widetilde{C}^{\mu}\left(\gamma^{-h} x\right)\right| \leq c \gamma^{-\frac{5 h}{4}},
$$

where the upper label, $\mu$ indicates the continuous derivative (as opposed to $\partial^{\mu}$ that is the lattice one).

As consequence, $\widetilde{C}(x)=\lim _{h \rightarrow \infty} C_{s}\left(\gamma^{h} x ; 0\right)$, and $\widetilde{C}(x)=0$ if $|x| \geq \gamma / 2$.

Proof. We discuss the details for the former inequality only; for the latter the argument is similar. Consider the formula for the Fourier transform of the covariance $C_{h}$ given in (A.13) for $m=0$ : the idea is to replace the $h$-dependent cutoff function $F_{h}(p ; 0)$ with $u(p)$; and to replace $-\widehat{\Delta}(p)$ with $p^{2}$ and the interval of integration $Q$ with $\mathbb{R}^{2}$. We have to prove that the errors so generated are $O\left(\gamma^{-\vartheta h}\right)$ for some $\vartheta>0$. If $p \in Q,-\widehat{\Delta}(p) \geq\left(4 p^{2} / \pi^{2}\right)$ and $\left|p^{2}+\widehat{\Delta}(p)\right| \leq(\sqrt{2} / 3)|p|^{3}$; then, by the second of (A.21),

$$
\left|\frac{1}{-\widehat{\Delta}(p)}-\frac{1}{p^{2}}\right| F_{h}\left(\gamma^{h} p ; 0\right) \leq \frac{C}{|p|} \frac{1}{1+\left|\gamma^{h} p\right|^{4}} .
$$

The replacement of $\widehat{\Delta}(p)$ with $p^{2}$ in $C_{s}$ gives an error not larger than the integral of (A.26) over $\mathbb{R}^{2}$, which is $\mathrm{O}\left(\gamma^{-h}\right)$. Add and subtract $u\left(\gamma^{h} p\right)$ and $u\left(\gamma^{h+1} p\right)$ in place of $F_{h}\left(\gamma^{h} p ; 0\right)$ and $F_{h+1}\left(\gamma^{h+1} p ; 0\right)$, respectively, to get

$$
\begin{aligned}
C_{s}(x) & =\int_{Q} \frac{d^{2} p}{(2 \pi)^{2}} e^{i x p} \frac{u\left(\gamma^{h} p\right)-u\left(\gamma^{h+1} p\right)}{p^{2}}+\int_{Q} \frac{d^{2} p}{(2 \pi)^{2}} e^{i x p} \frac{F_{h}\left(\gamma^{h} p ; 0\right)-u\left(\gamma^{h} p\right)}{p^{2}} \\
& -\int_{Q} \frac{d^{2} p}{(2 \pi)^{2}} e^{i x p} \frac{F_{h+1}\left(\gamma^{h+1} p ; 0\right)-u\left(\gamma^{h+1} p\right)}{p^{2}}+\mathrm{O}\left(\gamma^{-h}\right) .
\end{aligned}
$$

Now assume that (proof is below)

$$
\int_{Q_{h}} \frac{d^{2} p}{(2 \pi)^{2}} \frac{\left|F_{h}(p ; 0)-u(p)\right|}{p^{2}} \leq c(\gamma) \gamma^{-\frac{h}{4}}
$$

(A.27) and (A.28) directly give (A.25). We are only left with proving assumption (A.28), that is a consequence of

$$
\int_{Q_{h^{\prime}}} \frac{d^{2} p}{(2 \pi)^{2}} \frac{\left|F_{h}(p ; 0)-F_{h+1}(p ; 0)\right|}{p^{2}} \leq c(\gamma) \gamma^{-\frac{h}{4}},
$$


for any $h>h^{\prime}>0$. Define

$$
\begin{aligned}
& O_{h}(p):=\prod_{n=1}^{h}\left|\widehat{A}_{h, n}(p ; 0)\right|^{2}-\prod_{n=0}^{h}\left|\widehat{A}_{h+1, n+1}(p ; 0)\right|^{2} \\
& =\sum_{m=1}^{h}\left[\left|\widehat{A}_{h, m}(p ; 0)\right|^{2}-\left|\widehat{A}_{h+1, m+1}(p ; 0)\right|^{2}\right] \prod_{n=1}^{m-1}\left|\widehat{A}_{h, n}(p ; 0)\right|^{2} \prod_{n=m+1}^{h}\left|\widehat{A}_{h+1, n+1}(p ; 0)\right|^{2} \\
& +\left[1-\left|\widehat{A}_{h+1,1}(p ; 0)\right|^{2}\right] \prod_{n=1}^{h}\left|\widehat{A}_{h+1, n+1}(p ; 0)\right|^{2}:=\sum_{m=0}^{h} O_{h, m}(p)
\end{aligned}
$$

then, using (A.17), (A.18), (A.19) and (A.20), for $0 \leq m \leq h$,

$$
\left|O_{h, m}(p)\right| \leq C(\gamma) \frac{\min \left\{\gamma^{-m},|p| \gamma^{-(h-m)}\right\}}{1+p^{4}} ;
$$

therefore

$$
\sum_{m=1}^{h+1} \int \frac{d^{2} p}{(2 \pi)^{2}} \frac{\left|O_{h, m}(p)\right|}{p^{2}} \leq C(\gamma) \gamma^{-\frac{h}{2}} h
$$

(when $m \leq h / 2$, use the bound $|p| \gamma^{-(h-m)}$; when $m>h / 2$, use the bound $|p| \gamma^{-(h-m)}$ for the integration over $|p| \leq \gamma^{-h / 2}$ and the bound $\gamma^{-m}$ for the integration over $\gamma^{-h / 2} \leq|p| \leq 1$ and $|p| \geq 1$ ). This proves (A.29).

\section{A.3.1 Proof of (3.4)}

Integrating in polar coordinates, only using that $u(0)=1$ and $\lim _{\rho \rightarrow \infty} u(\rho \cos \vartheta, \rho \sin \vartheta)=$ 0

$$
\widetilde{C}(0)=\int \frac{d^{2} p}{(2 \pi)^{2}} \frac{u(p)-u(\gamma p)}{p^{2}}=\frac{1}{2 \pi} \ln \gamma
$$

(3.4) follows.

\section{A.3.2 Proof of Lemma 3.1}

A preliminary result is that, for large $|x|$,

$$
\widetilde{\Gamma}_{\infty, 0}(x \mid 0):=\int \frac{d^{2} p}{(2 \pi)^{2}}\left(e^{i p x}-1\right) \frac{u(p)}{p^{2}}=-\frac{1}{2 \pi} \ln |x|+c+o(1) .
$$

This formula is an easy consequence of the decomposition of the integral into three parts: for $I_{1}=\left\{|p| \leq|x|^{-1}\right\}$ and $I_{2}=\left\{|p|>|x|^{-1}\right\}$, the above integral is equal to

$$
\int_{I_{1}} \frac{d^{2} p}{(2 \pi)^{2}}\left(e^{i p x}-1\right) \frac{u(p)}{p^{2}}+\int_{I_{2}} \frac{d^{2} p}{(2 \pi)^{2}} e^{i p x} \frac{u(p)}{p^{2}}-\int_{I_{2}} \frac{d^{2} p}{(2 \pi)^{2}} \frac{u(p)}{p^{2}} ;
$$


the only term that is divergent for large $|x|$ is the third, and its leading term can be explicitly computed. Now consider the coefficient $a_{j}$. Let $\widetilde{w}_{b, j}$ and $\widetilde{\Gamma}_{j}(0 \mid y)$ be the same function defined in Sec 4.2 , but with $\widetilde{C}\left(\gamma^{-h} x\right)$ in place of $C_{s}(x)$. It is not difficult to see, also using (A.25), that an equivalent formula for the coefficient $a_{j}$ is, up to $O\left(L^{-\frac{j}{4}}\right)$ errors,

$$
\begin{aligned}
& \frac{\alpha^{2}}{2} \int d^{2} y y^{2}\left[\widetilde{w}_{b, j}(y)\left(e^{-\alpha^{2} \widetilde{\Gamma}_{j}(0 \mid y)}-1\right)+e^{-\alpha^{2} \widetilde{\Gamma}_{j}(0)}\left(e^{\alpha^{2} \widetilde{\Gamma}_{j}(y)}-1\right) L^{-4 j}\right] \\
& =\frac{\alpha^{2}}{2} \int d^{2} y y^{2}\left[\sum_{n=0}^{j} R_{n}^{(j)}(y)-\sum_{n=0}^{j-1} R_{n}^{(j-1)}(y)\right]
\end{aligned}
$$

for

$$
R_{n}^{(j-1)}(y):=e^{-\alpha^{2} \widetilde{\Gamma}_{j-1, n+1}(0 \mid y)} e^{-\alpha^{2} \widetilde{\Gamma}_{n}(0)}\left(e^{\alpha^{2} \widetilde{\Gamma}_{n}(y)}-1\right) L^{-4 n} .
$$

From now on we only consider the case $\alpha^{2}=8 \pi$. Since $R_{n}^{(j-1)}(y)=L^{4} R_{n+1}^{(j)}(y L)$, (A.33) becomes

$$
\begin{aligned}
\frac{\alpha^{2}}{2} \int d^{2} y y^{2} R_{0}^{(j)}(y) & =\frac{\alpha^{2}}{2} \int d^{2} y y^{2} e^{-\alpha^{2} \widetilde{\Gamma}_{\infty, 1}(0 \mid y)} e^{-\alpha^{2} \widetilde{\Gamma}_{0}(0)}\left(e^{-\alpha^{2} \widetilde{\Gamma}_{0}(y)}-1\right)+O\left(L^{-j}\right) \\
& =\frac{\alpha^{2}}{2} \int \frac{d^{2} y}{y^{2}}\left[w(y)-w\left(y L^{-1}\right)\right]+O\left(L^{-j}\right)
\end{aligned}
$$

for $w(y)=y^{4} e^{-\alpha^{2} \widetilde{\Gamma}_{\infty, 0}(0 \mid y)}$ and the new error $O\left(L^{-j}\right)$ in the first line is due to the replacement of $\widetilde{\Gamma}_{j, 1}(0 \mid y)$ with $\widetilde{\Gamma}_{\infty, 1}(0 \mid y)$. The last integral can be computed in polar coordinates only using the fact that $w(0)=0$ and, by (A.32), $\lim _{y \rightarrow \infty} w(y)=e^{c}$. This proves the first of (3.15).

Now consider the coefficient $b_{j}\left(\right.$ still $\left.\alpha^{2}=8 \pi\right)$. With the same argument used for $a_{j}$, an equivalent formula for $b_{j}$ is, up to an error term $O\left(L^{-\frac{j}{4}}\right)$,

$$
\frac{\alpha^{2}}{2} \sum_{\mu=\widehat{e}} \int d^{2} y\left[\left(\widetilde{\Gamma}_{j, 0}^{, \mu}\right)^{2}(y)-\left(\widetilde{\Gamma}_{j-1,0}^{\mu}\right)^{2}(y)\right] .
$$

As $\widetilde{\Gamma}_{j-1,0}^{, \mu}(y)=L \widetilde{\Gamma}_{j, 1}^{, \mu}(y L),(\underline{A .35})$ becomes, up another error term $O\left(L^{-j}\right)$,

$$
\begin{aligned}
& \frac{\alpha^{2}}{2} \sum_{\mu=\widehat{e}} \int d^{2} y\left[2 \widetilde{\Gamma}_{\infty, 1}^{, \mu}(y) \widetilde{\Gamma}_{0}^{, \mu}(y)+\left(\widetilde{\Gamma}_{0}^{, \mu}\right)^{2}(y)\right] \\
& =\frac{\alpha^{2}}{2} \sum_{\mu=\widehat{e}} \int d^{2} y\left[\left(\widetilde{\Gamma}_{\infty, 0}^{, \mu}\right)^{2}(y)-\left(\widetilde{\Gamma}_{\infty, 1}^{, \mu}\right)^{2}(y)\right] \\
& =\frac{\alpha^{2}}{2} \int \frac{d^{2} p}{(2 \pi)^{2}} \frac{[u(\rho)]^{2}-[u(L \rho)]^{2}}{p^{2}} .
\end{aligned}
$$

This proves the second of (3.15). 


\section{B Charged Components}

The following constructions was introduced in [14]. By iteration on scale $j$, the polymer activities are showed to satisfy, for any $m \in \mathbb{Z}$,

$$
K_{j}\left(\varphi+\frac{2 m \pi}{\alpha}, X\right)=K_{j}(\varphi, X) .
$$

Accordingly, given $X \in \mathcal{P}_{j}^{c}$ and a field $\left\{\varphi_{x}\right\}_{x \in X}$, the function of real variable $F(t):=$ $K_{j}(\varphi+t, X)$ is smooth and periodic of period $2 \pi / a$ : expand $F(t)$ in (absolutely convergent) Fourier series and, at $t=0$, obtain (4.2) with charged components

$$
\widehat{K}_{j}(q, \varphi, X):=\frac{\alpha}{2 \pi} \int_{0}^{\frac{2 \pi}{\alpha}} d s K_{j}(\varphi+t, X) e^{-i q \alpha s} .
$$

Besides, by properties of the norms and since $G_{j}(\varphi, X)$ only depends upon the derivatives of $\varphi$,

$$
\left\|\widehat{K}_{j}(q, \varphi, X)\right\|_{h, T_{j}(\varphi, X)} \leq\left\|K_{j}(X)\right\|_{h, T_{j}(X)} G_{j}(\varphi, X) .
$$

\section{Lowest Orders Computation}

\section{C.1 First Order}

Let $B \in \mathcal{B}_{j}$. An explicit computation of $\mathbb{E}_{j}\left[V_{j}(\varphi, B)\right]$ yields

$$
\begin{gathered}
\frac{s_{j}}{2} \sum_{\substack{x \in B \\
\mu \in \widehat{e}}}\left(\partial^{\mu} \varphi^{\prime}\right)_{x}^{2}-|B| \frac{s_{j}}{2} \sum_{\mu \in \widehat{e}}\left(\partial^{\mu} \partial^{\mu} \Gamma_{j}\right)(0)+z_{j} L^{-2 j} e^{-\frac{\alpha^{2}}{2} \Gamma_{j}(0)} \sum_{\substack{x \in B \\
\sigma \in \pm}} e^{i \sigma \alpha \varphi_{x}^{\prime}} \\
\quad=-\frac{s_{j}}{2}|B| \sum_{\mu \in \widehat{e}}\left(\partial^{\mu} \partial^{\mu} \Gamma_{j}\right)(0)+V_{j+1}\left(s_{j}, L^{2} e^{-\frac{\alpha^{2}}{2} \Gamma_{j}(0)} z_{j}, \varphi^{\prime}, B\right) .
\end{gathered}
$$

\section{C.2 Second Order}

Notice the following "cancellations":

$$
\begin{gathered}
\sum_{y}\left(\partial^{\mu} \partial^{\nu} \Gamma_{j}(y)\right)=-\left.k^{\mu} k^{\nu} \widehat{\Gamma}_{j}(k)\right|_{k=0}=0 \\
\sum_{y} e^{-\alpha^{2} \Gamma_{j}(0)}\left(e^{\alpha^{2} \Gamma_{j}(y)}-1\right) y^{\mu} y^{\nu}=\delta^{\mu \nu} \sum_{y} e^{-\alpha^{2} \Gamma_{j}(0)}\left(e^{\alpha^{2} \Gamma_{j}(y)}-1\right) \frac{|y|^{2}}{2} .
\end{gathered}
$$


Let $D=\bar{B}$, and $B \in \mathcal{B}_{j}$. An explicit computation yields:

$$
\begin{aligned}
\frac{1}{2} E_{j}^{T}\left[V_{j}(\varphi, B) ; V_{j}\left(\varphi, D^{*}\right)\right]= & s_{j}^{2} F_{a, j+1}\left(\varphi^{\prime}, B\right)+z_{j}^{2} F_{b, j+1}\left(\varphi^{\prime}, B\right) \\
& +z_{j}^{2} F_{c, j+1}\left(\varphi^{\prime}, B\right)+z_{j} s_{j} F_{d, j+1}\left(\varphi^{\prime}, B\right)
\end{aligned}
$$

where we have defined:

$$
\begin{aligned}
& F_{a, j+1}\left(\varphi^{\prime}, B\right):=-\frac{1}{2} \sum_{y \in \mathbb{Z}^{2}} \sum_{\substack{\mu \in \widehat{Q} \\
\nu \in \widehat{e}}}\left(\partial^{\mu} \partial^{\nu} \Gamma_{j}\right)(y) \sum_{x \in B}\left(\partial^{\mu} \varphi_{x}^{\prime}\right)\left[\left(\partial^{\nu} \varphi_{x+y}^{\prime}\right)-\left(\partial^{\nu} \varphi_{x}^{\prime}\right)\right] \\
& +|B| \frac{1}{4} \sum_{y \in \mathbb{Z}^{2}} \sum_{\substack{\mu \in \hat{e} \\
\nu \in \hat{e}}}\left(\partial^{\mu} \partial^{\nu} \Gamma_{j}\right)(y)\left(\partial^{\mu} \partial^{\nu} \Gamma_{j}\right)(y \mid 0) \\
& F_{b, j+1}\left(\varphi^{\prime}, B\right):=\sum_{y \in \mathbb{Z}^{2}} \frac{1}{2} e^{-\alpha^{2} \Gamma_{j}(0)}\left(e^{\alpha^{2} \Gamma_{j}(y)}-1\right) L^{-4 j} . \\
& \cdot \sum_{\substack{x \in B \\
\sigma= \pm}}\left[e^{i \sigma \alpha\left(\varphi_{x}^{\prime}-\varphi_{x+y}^{\prime}\right)}-1+\frac{\alpha^{2}}{2} \sum_{\substack{\mu \in \hat{\bigotimes} \\
\nu \in \hat{e}}}\left(\partial^{\mu} \varphi_{x}^{\prime}\right)\left(\partial^{\nu} \varphi_{x}^{\prime}\right) y^{\mu} y^{\nu}\right] \\
& +\sum_{y} e^{-\alpha^{2} \Gamma_{j}(0)}\left(e^{\alpha^{2} \Gamma_{j}(y)}-1\right) L^{-4 j}|B| \\
& -\sum_{y \in \mathbb{Z}^{2}} \frac{\alpha^{2}}{2} e^{-\alpha^{2} \Gamma_{j}(0)}|y|^{2}\left(e^{\alpha^{2} \Gamma_{j}(y)}-1\right) L^{-4 j} \frac{1}{2} \sum_{x \in B} \sum_{\mu \in \widehat{e}}\left(\partial^{\mu} \varphi_{x}^{\prime}\right)^{2} \\
& F_{c, j+1}\left(\varphi^{\prime}, B\right):=\frac{L^{-4 j}}{2} \sum_{y \in \mathbb{Z}^{2}} e^{-\alpha^{2} \Gamma_{j}(0)}\left(e^{-\alpha^{2} \Gamma_{j}(y)}-1\right) \sum_{x \in B} \sum_{\varepsilon} e^{i \sigma \alpha\left(\varphi_{x}^{\prime}+\varphi_{x+y}^{\prime}\right)} \\
& F_{d, j+1}\left(\varphi^{\prime}, B\right):=\frac{L^{-2 j}}{2} \sum_{\substack{y \in \mathbb{Z}^{2} \\
\nu \in \widehat{e}}} e^{-\frac{\alpha^{2}}{2} \Gamma_{j}(0)}\left(\partial^{\nu} \Gamma_{j}\right)(y) . \\
& \cdot \sum_{\substack{x \in B \\
\sigma= \pm}} i \sigma \alpha\left[e^{i \sigma \alpha \varphi_{x}^{\prime}}\left(\partial^{\nu} \varphi_{x+y}^{\prime}\right)-e^{i \sigma \alpha \varphi_{x+y}^{\prime}}\left(\partial^{\nu} \varphi_{x}^{\prime}\right)\right] \\
& -\frac{L^{-2 j}}{4} \sum_{\substack{y \in \mathbb{Z}^{2} \\
\nu \in \widehat{e}}} e^{-\frac{\alpha^{2}}{2} \Gamma_{j}(0)}\left(\partial^{\nu} \Gamma_{j}\right)^{2}(y) \alpha^{2} \sum_{\substack{x \in B \\
\sigma= \pm}}\left(e^{i \sigma \alpha \varphi_{x+y}^{\prime}}-e^{i \sigma \alpha \varphi_{x}^{\prime}}\right) \\
& -\frac{L^{-2 j}}{2} \sum_{\substack{y \in \mathbb{Z}^{2} \\
\nu \in \widehat{e}}} e^{-\frac{\alpha^{2}}{2} \Gamma_{j}(0)}\left(\partial^{\nu} \Gamma_{j}\right)^{2}(y) \alpha^{2} \sum_{\substack{x \in B \\
\sigma \in \pm}} e^{i \sigma \alpha \varphi_{x}^{\prime}}
\end{aligned}
$$


Accordingly, by the definitions of $W_{m, j}$ in (4.25),

$$
\begin{aligned}
& W_{a, j+1}\left(\varphi^{\prime}, B\right)+L^{-2 j} \widehat{e}_{3, j}|B|=F_{a, j+1}\left(\varphi^{\prime}, B\right)+\mathbb{E}_{j}\left[W_{a, j}\left(\varphi^{\prime}, B\right)\right] \\
& W_{b, j+1}\left(\varphi^{\prime}, B\right)+L^{-2 j} \widehat{e}_{4, j}|B|+V_{j+1}\left(-a_{j}, 0, \varphi^{\prime}, B\right)=F_{b, j+1}\left(\varphi^{\prime}, B\right)+\mathbb{E}_{j}\left[W_{b, j}(\varphi, B)\right] \\
& W_{c, j+1}\left(\varphi^{\prime}, B\right)=F_{c, j+1}\left(\varphi^{\prime}, B\right)+\mathbb{E}_{j}\left[W_{c, j}(\varphi, B)\right] \\
& W_{d, j+1}\left(\varphi^{\prime}, B\right)+V_{j+1}\left(0,-L^{2} e^{-\frac{\alpha^{2}}{2} \Gamma_{j}(0)} b_{j}, \varphi^{\prime}, B\right)=F_{d, j+1}\left(\varphi^{\prime}, B\right)+\mathbb{E}_{j}\left[W_{d, j}(\varphi, B)\right]
\end{aligned}
$$

where $\widehat{e}_{3, j}$ and $\widehat{e}_{4, j}$ are defined in (4.24).

\section{C.3 Estimates for $W_{j}$}

Let $B \in \mathcal{B}_{j}$. If $|y| \leq L^{n+1} / 2$, uniformly in $j$

$$
\begin{gathered}
\left|\Gamma_{j-1, n+1}(0)-\Gamma_{j-1, n+1}(y)\right| \leq C, \\
\left|\Gamma_{j-1, n+1}(0)+\Gamma_{j-1, n+1}(y)-(j-n-1) \frac{1}{\pi} \ln L\right| \leq C .
\end{gathered}
$$

Accordingly,

$$
\begin{gathered}
L^{-j} \sum_{y \in \mathbb{Z}^{2}}\left|w_{a, j}^{\mu \nu}(y)\right||y| \leq C(L), \quad L^{-j} \sum_{y \in \mathbb{Z}^{2}}\left|w_{b, j}^{\mu \nu}(y)\right||y|^{3} \leq C(L), \\
L^{2 j} \sum_{y \in \mathbb{Z}^{2}}\left|w_{c, j}(y)\right| \leq C(L), \quad L^{j} \sum_{y \in \mathbb{Z}^{2}}\left|w_{d, j}^{\mu}(y)\right| \leq C(L), \\
L^{j} \sum_{y \in \mathbb{Z}^{2}}\left|w_{e, j}(y)\right||y| \leq C(L) .
\end{gathered}
$$

The above inequalities yield

$$
\begin{aligned}
& \left\|W_{a, j}(s, z, \varphi, B)\right\|_{h, T_{j}(\varphi, B)} \\
& \leq s^{2} \sum_{\mu, \nu, \rho \in e} \sum_{y \in \mathbb{Z}^{2}}\left|w_{a, j}^{\mu \nu}(y)\right|\left|y^{\rho}\right||B| \sup _{x, z \in B}\left\|\left(\partial^{\mu} \varphi_{x}\right)\left(\partial^{\rho} \partial^{\nu} \varphi_{z}\right)\right\|_{h, T_{j}(\varphi, B)} \\
& \leq s^{2} C(L)\left(1+\max _{n=1,2}\left\|\nabla_{j}^{n} \varphi\right\|_{L^{\infty}\left(B^{*}\right)}^{2}\right)
\end{aligned}
$$




$$
\begin{aligned}
& \left\|W_{b, j}(s, z, \varphi, B)\right\|_{h, T_{j}(\varphi, B)} \leq z^{2} C(\alpha, L) \sum_{y \in \mathbb{Z}^{2}}\left|w_{b, j}(y)\right||y|^{3} \cdot \\
& \cdot|B| \sum_{\mu, \nu, \rho=\widehat{e}} \sup _{x, z \in B}\left[\left\|\left(\partial^{\mu} \varphi_{z}\right)^{3}+\left(\partial^{\rho} \partial^{\mu} \varphi_{x}\right)\left(\partial^{\nu} \varphi_{z}\right)\right\|_{h, T_{j}(\varphi, B)}\right] \\
& \leq z^{2} C(\alpha, L)\left(1+\max _{n=1,2}\left\|\nabla_{j}^{n} \varphi\right\|_{L^{\infty}\left(B^{*}\right)}^{2}\right) \\
& \left\|W_{c, j}(s, z, \varphi, B)\right\|_{h, T_{j}(\varphi, B)} \leq z^{2} C(\alpha) \sum_{y \in \mathbb{Z}^{2}}\left|w_{c, j}(y)\right||B| \leq z^{2} C(L, \alpha) \\
& \left\|W_{d, j}(s, z, \varphi, B)\right\|_{h, T_{j}(\varphi, B)} \leq|z s| C(\alpha) \sum_{\mu} \sum_{y \in \mathbb{Z}^{2}}\left[\left|w_{d, j}^{\mu}(y)\right|+\left|w_{e, j}(y) \| y\right|\right] \\
& \cdot|B| \sup _{z \in B^{*}}\left\|\left(\partial^{\mu} \varphi_{z}\right)\right\|_{h, T_{j}(\varphi, B)} \\
& \leq|z s| C(\alpha, L)\left(1+\max _{n=1,2}\left\|\nabla_{j}^{n} \varphi\right\|_{L^{\infty}\left(B^{*}\right)}^{2}\right)
\end{aligned}
$$

All together these bounds give (6.34).

\section{Proof of Lemma 5.2 .}

The goal in to implement the idea of finer decomposition of the covariances, [7, in order to have control over the different field regulators needed for the Coulomb Gas. By (A.12), decompose the covariances and the field

$$
\Gamma_{j}(x)=\sum_{s \in I_{j, j+1}} C_{s}(x), \quad \zeta_{x}^{(j)}=\sum_{s \in I_{j, j+1}} \xi_{x}^{(s)},
$$

so that $\xi_{x}^{(s)}$ is a Gaussian random field with covariance $E_{s}\left[\xi_{x}^{(s)} \xi_{y}^{(s)}\right]=C_{s}(x-y)$, with range $L^{s+\log _{L} \gamma} / 2=L^{j} \gamma^{m+1} / 2$ and typical momentum $L^{-s}=L^{-j} \gamma^{-m}$. The notation for the fields is now

$$
\varphi_{x}^{(s)}=\varphi_{x}^{\left(s^{\prime}\right)}+\xi_{x}^{(s)}
$$

for $s^{\prime}=s+\log _{L} \gamma$. It is clear how to extend previous definitions to have $s$-blocks, $s$-polymers, $L_{s}^{2}$ norms and $G_{s}$ field regulators. We use $\bar{X}_{s}$ to indicate the closure of the set $X$ on scale $s$ (hence, for any $j$-polymer, $X \bar{X}_{j} \equiv X$ and $\bar{X}_{j+1} \equiv \bar{X}$ ). Given $c_{4}>0$, define $(c f r$. (5.10) $)$

$$
\ln g_{s}\left(\xi, \bar{X}_{s}\right)=c_{4} \kappa_{L} \sum_{n=0,1,2} W_{s}\left(\nabla_{s}^{n} \xi, \bar{X}_{s}\right)^{2} .
$$


Lemma D.1 There exists a choice of the constants $c_{1}, c_{3}, c_{4}$ and $\gamma$ such that:

1) for any $X \in \mathcal{P}_{s}^{c}$,

$$
G_{s}\left(\varphi^{(s)}, \bar{X}_{s}\right) \leq G_{s^{\prime}}\left(\varphi^{\left(s^{\prime}\right)}, \bar{X}_{s^{\prime}}\right) g_{s}\left(\xi^{(s)}, \bar{X}_{s}\right)
$$

2) for any $X \in \mathcal{S}_{s}$,

$$
G_{s}\left(\varphi^{(s)}, \bar{X}\right) \leq G_{s^{\prime}}\left(\varphi^{\left(s^{\prime}\right)}, \bar{X}_{s^{\prime}}\right)^{2 / 3} g_{s}\left(\xi^{(s)}, \bar{X}_{s}\right) .
$$

Proof. Only in this proof, let us shorten the notations $\varphi^{(s)}, \varphi^{\left(s^{\prime}\right)}, \xi^{(s)}, \bar{X}_{s}$ and $\bar{X}_{s^{\prime}}$ into $\varphi, \varphi^{\prime}, \xi, X$ and $\bar{X}$, respectively. Since $\varphi=\varphi^{\prime}+\xi$, for any $0<\alpha<1$, by discreet partial integration (see (6.117) of [5])

$$
\begin{aligned}
\left\|\nabla_{s} \varphi\right\|_{L_{s}^{2}(X)}^{2} \leq & \left\|\nabla_{s} \varphi^{\prime}\right\|_{L_{s}^{2}(X)}^{2}+\alpha\left\|\nabla_{s} \varphi^{\prime}\right\|_{L_{s}^{2}(\partial X)}^{2}+\alpha\left\|\nabla_{s}^{2} \varphi^{\prime}\right\|_{L_{s}^{2}(X)}^{2} \\
& +\left\|\nabla_{s} \xi\right\|_{L_{s}^{2}(X)}^{2}+\alpha^{-1}\|\xi\|_{L_{s}^{2}(\partial X)}^{2}+\alpha^{-1}\|\xi\|_{L_{s}^{2}(X)}^{2}
\end{aligned}
$$

whereas, by squaring triangular inequalities

$$
\begin{gathered}
\left\|\nabla_{s} \varphi\right\|_{L_{s}^{2}(\partial X)}^{2} \leq 2\left\|\nabla_{s} \varphi^{\prime}\right\|_{L_{s}^{2}(\partial X)}^{2}+2\left\|\nabla_{s} \xi\right\|_{L_{s}^{2}(\partial X)}^{2}, \\
W_{s}\left(\nabla_{s}^{2} \varphi, X\right)^{2} \leq 2 W_{s}\left(\nabla_{s}^{2} \varphi^{\prime}, X\right)^{2}+2 W_{s}\left(\nabla_{s}^{2} \xi, X\right)^{2} .
\end{gathered}
$$

Collecting the last three formulas, by (6.110) of [5],

$$
\begin{aligned}
\ln G_{s}(\varphi, X) \leq & c_{1} \kappa_{L}\left\|\nabla_{s} \varphi^{\prime}\right\|_{L_{s}^{2}(X)}^{2}+f_{3} \kappa_{L}\left\|\nabla_{s} \varphi^{\prime}\right\|_{L_{s}^{2}(\partial X)}^{2}+f_{1} \kappa_{L} W_{s}\left(\nabla_{s}^{2} \varphi^{\prime}, X\right)^{2} \\
& +f_{4} \kappa_{L} \sum_{n=0,1,2} W_{s}\left(\nabla_{s}^{n} \xi, X\right)^{2}
\end{aligned}
$$

for $f_{3}=\alpha c_{1}+2 c_{3}, f_{1}=(4 \alpha+2) c_{1}<6 c_{1}$ and $f_{4}=4 c_{1} \alpha^{-1}+8 c_{3}+4 c_{1}$. In order to pass to scale $s^{\prime}$, use the inequality

$$
\left\|\nabla_{s} \varphi^{\prime}\right\|_{L_{s}^{2}(\partial X)}^{2} \leq\left\|\nabla_{s} \varphi^{\prime}\right\|_{L_{s}^{2}(\partial \bar{X})}^{2}+c\left\|\nabla_{s} \varphi^{\prime}\right\|_{L_{s}^{2}(\bar{X} \backslash X)}^{2}+c W_{s}\left(\nabla_{s}^{2} \varphi^{\prime}, \bar{X} \backslash X\right)^{2}
$$

for a certain constant $c>0$ (for the proof, $c f r$. the proof of (6.105) of [5]). If $\alpha$ and $c_{3}$ are so small that $c f_{3} \leq c_{1}$,

$$
\begin{aligned}
\ln G_{s}(\varphi, X) \leq & c_{1} \kappa_{L}\left\|\nabla_{s} \varphi^{\prime}\right\|_{L_{s}^{2}(\bar{X})}^{2}+f_{3} \kappa_{L}\left\|\nabla_{s} \varphi^{\prime}\right\|_{L_{s}^{2}(\partial \bar{X})}^{2}+6 c_{1} \kappa_{L} W_{s}\left(\nabla_{s}^{2} \varphi^{\prime}, \bar{X}\right)^{2} \\
& +f_{4} \kappa_{L} \sum_{n=0,1,2} W_{s}\left(\nabla_{s}^{n} \xi, X\right)^{2}
\end{aligned}
$$

Finally, to restore the old constant $c_{3}$ and $c_{1}$ in the 2nd and 3rd terms, observe that these two terms are irrelevant, i.e. passing to scale $s^{\prime}$ gives $\gamma^{-1}$ 's factors:

$$
\left\|\nabla_{s} \varphi^{\prime}\right\|_{L_{s}^{2}(\partial \bar{X})}^{2} \leq \gamma^{-1}\left\|\nabla_{s^{\prime}} \varphi^{\prime}\right\|_{L_{s^{\prime}}^{2}(\partial \bar{X})}^{2}
$$




$$
W_{s}\left(\nabla_{s}^{2} \varphi^{\prime}, \bar{X}\right)^{2} \leq \gamma^{-2} W_{s^{\prime}}\left(\nabla_{s^{\prime}}^{2} \varphi^{\prime}, \bar{X}\right)^{2} ;
$$

the first term is, instead, scale invariant (and the fourth is relevant but there is no need to scale it). Therefore, for $c_{4} \geq f_{4}$, if $\gamma$ is so large that $f_{3} \gamma^{-1}<c_{3}$ and $6 \gamma^{-2}<1$ (D.4) is proven.

Then consider the case when $X \in \mathcal{S}_{s}$. If $\gamma>8$, it is possible to find the polymer $T X$, a translation of $X$, such that $T X$ is disjoint from $X$, but $(T X)^{*} \cap X^{*} \neq \emptyset$ and $T X \subset \bar{X}$; by (6.129) of [5], setting $X_{T}=T X \cup X$ and for a $c>0$,

$$
\frac{3}{2}\left\|\nabla_{s} \varphi^{\prime}\right\|_{L_{s}^{2}(X)}^{2} \leq\left\|\nabla_{s} \varphi^{\prime}\right\|_{L_{s}^{2}\left(X_{T}\right)}^{2}+c W_{s}\left(\nabla^{2} \varphi^{\prime} ; X_{T}\right)^{2} ;
$$

besides, as $X$ and $T X$ have disjoint boundaries,

$$
\left\|\nabla_{s} \varphi^{\prime}\right\|_{L_{s}^{2}(\partial X)}^{2} \leq\left\|\nabla_{s} \varphi^{\prime}\right\|_{L_{s}^{2}\left(\partial X_{T}\right)}^{2}, \quad W_{s}\left(\nabla_{s}^{2} \varphi^{\prime}, X\right)^{2} \leq W_{s}\left(\nabla_{s}^{2} \varphi^{\prime}, X_{T}\right)^{2} .
$$

Multiply both sides of $(\overline{\mathrm{D} .9})$ times $3 / 2$; then use (D.13) and (D.14) to obtain

$$
\begin{aligned}
\frac{3}{2} \ln G_{s}(\varphi, X) \leq & c_{1} \kappa_{L}\left\|\nabla_{s} \varphi^{\prime}\right\|_{L_{s}^{2}\left(X_{T}\right)}^{2}+\frac{3 f_{3}}{2} \kappa_{L}\left\|\nabla_{s} \varphi^{\prime}\right\|_{L_{s}^{2}\left(\partial X_{T}\right)}^{2} \\
& +t_{1} \kappa_{L} W_{s}\left(\nabla_{s}^{2} \varphi^{\prime}, X_{T}\right)^{2}+c_{4} \sum_{n=0,1,2} W_{s}\left(\nabla_{s}^{n} \xi, X\right)^{2}
\end{aligned}
$$

for $t_{1}=3 f_{1} / 2+c c_{1}$ and $c_{4}=3 f_{4} / 2$. This inequality is of the same form as (D.9), except for the fact that $X$ has been replaced by $X_{T}$ in the first three terms of the r.h.s, and the constants are different. Therefore one can proceed as done from (D.9) to obtain (D.4): since by construction $\bar{X}_{T}=\bar{X}$, (D.5) follows for $\alpha$ and $c_{3}$ small enough, and $\gamma$ large enough

To conclude the proof of Lemma [5.2, we need two results of [5].

Lemma D.2 If $\kappa_{L}$ is small enough (w.r.t $\gamma^{-2}$ ), for any $X \in \mathcal{P}_{s}$

$$
\mathbb{E}_{s}\left[g_{s}\left(\xi^{(s)}, X\right)\right] \leq e^{c \kappa_{L}|X|_{s}},
$$

where $|X|_{s}$ is the number of $s$-blocks in $X$.

Lemma D.3 For $X \in \mathcal{P}_{j}$

$$
\left(1+\max _{n=1,2}\left\|\nabla_{j+1}^{n} \varphi^{(j+1)}\right\|_{L^{\infty}\left(X^{*}\right)}\right)^{3} \leq \frac{c}{\kappa_{L}^{3 / 2}}\left[G_{j+1}\left(\varphi^{(j+1)}, \bar{X}\right)\right]^{1 / 3} .
$$


The former is Lemma 6.31 of [5] and its hypothesis is fulfilled if $M$ is large enough; the latter corresponds to formula (6.127) of [5]. Use (D.4) iteratively, then (D.16), to find, for any $X \in \mathcal{P}_{j}$,

$$
\begin{aligned}
\mathbb{E}_{j}\left[G_{j}\left(\varphi^{(j)}, X\right)\right] & \leq G_{j+1}\left(\varphi^{(j+1)}, \bar{X}\right) \prod_{s \in I_{j, j+1}} \mathbb{E}_{s}\left[g_{s}\left(\xi^{(s)}, \bar{X}_{s}\right)\right] \\
& \leq G_{j+1}\left(\varphi^{(j+1)}, \bar{X}\right) e^{c \kappa_{L} \sum_{s \in I_{j, j+1}}\left|\bar{X}_{s}\right|_{s}}
\end{aligned}
$$

This, for $\gamma$ large enough proves (5.16) for $\left|\bar{X}_{s}\right|_{s} \leq|X|_{j}$ and $c \kappa_{L}=C / \ln L=$ $C\left(\log _{\gamma} e\right) / M$. In the same fashion, using by (D.5) iteratively, and then (D.16), (D.17), for any $X \in \mathcal{S}_{j}$ (so that $\bar{X}_{s} \in \mathcal{S}_{s}$ for any $s$ ),

$$
\begin{aligned}
& \left(1+\max _{n=1,2}\left\|\nabla_{j+1}^{n} \varphi^{(j+1)}\right\|_{L^{\infty}\left(X^{*}\right)}\right)^{3} \mathbb{E}_{j}\left[G_{j}\left(\varphi^{(j)}, X\right)\right] \\
& \leq\left(1+\max _{n=1,2}\left\|\nabla_{j+1}^{n} \varphi^{(j+1)}\right\|_{L^{\infty}\left(X^{*}\right)}\right)^{3} G_{j+1}^{2 / 3}\left(\varphi^{(j+1)}, \bar{X}\right) \prod_{s \in I_{j, j+1}} \mathbb{E}_{s}\left[g_{s}\left(\xi^{(s)}, \bar{X}_{s}\right)\right] \\
& \leq \frac{c}{\kappa_{L}^{3 / 2}} G_{j+1}\left(\varphi^{(j+1)}, \bar{X}\right) e^{c \kappa_{L} \sum_{s \in I_{j, j+1}}\left|\bar{X}_{s}\right|_{s}} .
\end{aligned}
$$

This proves (5.17). (When $G_{j}\left(\varphi^{(j)}, X\right)$ is replaced by $\sup _{t \in[0,1]} G_{j}\left(t \varphi^{(j+1)}+\zeta, X\right)$, only little changes are required.)

\section{E Proof of Theorems 5.5, 5.6 and 5.7}

For $F \in \mathcal{N}_{j}(X)$ and $m$ a positive integer, define

$$
\|F(\varphi)\|_{h, T_{j}^{\geq m}(\varphi, X)}:=\sum_{n \geq m} \frac{h^{n}}{n !}\|F(\varphi)\|_{T_{j}^{n}(\varphi, X)}
$$

(it corresponds to (5.5) when $m=0$ ).

Lemma E.1 Let $F \in \mathcal{N}_{j}(X)$ and $X \in \mathcal{P}_{j}$ :

a) if $X \in \mathcal{S}_{j}$ and $x_{0} \in X$, for $(\delta \varphi)_{x}:=\varphi_{x}-\varphi_{x_{0}}$ and $\rho=5 L^{-1}$,

$$
\|F(\delta \varphi)\|_{h, T_{j+1}(\varphi, X)} \leq\left.\|F(\xi)\|_{\rho h, T_{j}(\xi, X)}\right|_{\xi_{x}=\delta \varphi_{x}} ;
$$

b) given $\psi \in \mathcal{C}_{j}^{2}(X)$, if $\Delta:=\|\psi\|_{\mathcal{C}_{j}^{2}(X)}$ is finite,

$$
\|F(\varphi+\psi)\|_{h, T_{j}(\varphi, X)} \leq\|F(\varphi)\|_{h+\Delta, T_{j}(\varphi, X)} ;
$$


c) for $m=0,2$ and any $h>0$,

$$
\left\|\operatorname{Rem}_{m} F(\xi)\right\|_{h, T_{j}(\xi, X)} \leq\left(1+h^{-1}\|\xi\|_{\mathcal{C}_{j}^{2}(X)}\right)^{m} \sup _{t \in[0,1]}\|F(t \xi)\|_{h, T_{j}^{\geq m}(t \xi, X)} .
$$

where the Taylor remainder is in the field $\xi$.

Proof. (E.2) follows from the identity

$$
\sum_{x \in X^{*}} f_{x} \frac{\partial F(\delta \varphi)}{\partial \varphi_{x}}=\left.\sum_{x \in X^{*}}(\delta f)_{x} \frac{\partial F}{\partial \varphi_{x}}(\xi)\right|_{\xi=\delta \varphi}
$$

and the fact that, for $X$ small, $\|\delta f\|_{\mathcal{C}_{j}^{2}(X)} \leq 5 L^{-1}\|f\|_{\mathcal{C}_{j+1}^{2}(X)}$. (E.3) is a direct consequence of Taylor series. Consider (E.4) for $m=2$ - the case $m=0$ can be proved with similar arguments - and set $O(\xi):=\operatorname{Rem}_{2} F(\xi)$; then using test functions $f_{1}, f_{2}, \ldots$ such that $\left\|f_{j}\right\|_{\mathcal{C}_{j}^{2}(X)} \leq 1$, for $n=0,1,2$

$$
\begin{aligned}
& \frac{h^{n}}{n !}\left|D_{\xi}^{n} O(\xi) \cdot\left(f_{1}, \ldots, f_{n}\right)\right| \\
& \quad \leq \frac{h^{n}}{n !} \int_{0}^{1} d t \frac{(1-t)^{2-n}}{(2-n) !}\left|D_{\xi}^{3} F(t \xi) \cdot\left(f_{1}, \ldots, f_{n}, \xi, \ldots, \xi\right)\right| \\
& \quad \leq \frac{3 !}{(3-n) ! n !}\left[h^{-1}\|\xi\|_{\mathcal{C}_{j}^{2}(X)}\right]^{3-n} \frac{h^{3}}{3 !} \sup _{t \in[0,1]}\|F(t \xi)\|_{h, T_{j}^{3}(t \xi, X)},
\end{aligned}
$$

whereas, for $n \geq 3$,

$$
\frac{h^{n}}{n !}\left|D_{\xi}^{n} O(\xi) \cdot\left(f_{1}, \ldots, f_{n}\right)\right| \leq \frac{h^{n}}{n !}\|F(\xi)\|_{h, T_{j}^{n}(\xi, X)} .
$$

The result follows by first summing the terms with $n=0,1,2,3$ with the binomial theorem, and then summing the terms with $n>3$.

\section{E.1 Proof of Theorem 5.6}

Consider the measure $\mathbb{E}_{j}$ and an integrable function $F(\varphi)$. Factorize the covariance into $\Gamma_{j}=g_{j} \circ g_{j}$ and call $\mathbb{E}_{I}$ the Gaussian expectation with covariance $I=\left(\delta_{i, j}\right)$; under the imaginary translation $\zeta_{x} \rightarrow \zeta_{x}+i\left(g_{j} f\right)_{x}$ where $f$ is any test function with finite support,

$$
\mathbb{E}_{j}[F(\varphi)]=\mathbb{E}_{I}\left[F\left(\varphi^{\prime}+\left(g_{j} \zeta\right)\right)\right]=e^{\frac{1}{2}\left(f, \Gamma_{j} f\right)} \mathbb{E}_{j}\left[e^{-i(\zeta, f)} F\left(\varphi+i\left(\Gamma_{j} f\right)\right)\right] .
$$

Now apply this identity with $F(\varphi)=\widehat{K}_{j}(q, \varphi, X)$ : calling $\psi_{x}:=\left(\Gamma_{j} f\right)_{x}$ and $(\delta \psi)_{x}:=$ $\left(\Gamma_{j} f\right)_{x}-\left(\Gamma_{j} f\right)_{x_{0}}$, by (4.3),

$$
\begin{aligned}
\mathbb{E}_{j}\left[\widehat{K}_{j}(q, \varphi, X)\right] & =e^{\frac{1}{2}\left(f, \Gamma_{j} f\right)} \mathbb{E}_{j}\left[e^{-i(\zeta, f)} \widehat{K}_{j}(q, \varphi+i \psi, X)\right] \\
& =e^{\frac{1}{2}\left(f, \Gamma_{j} f\right)-\alpha q\left(\delta_{x_{0}}, \Gamma_{j} f\right)} \mathbb{E}_{j}\left[\widehat{K}_{j}(q, \varphi+i \delta \psi, X)\right]
\end{aligned}
$$


where $\left(\delta_{x_{0}}\right)_{x}:=\delta_{x, x_{0}}$. Finally,

$$
\begin{aligned}
\| \mathbb{E}_{j} & {\left[\widehat{K}_{j}(q, \varphi, X)\right] \|_{h, T_{j+1}\left(\varphi^{\prime}, X\right)} } \\
& \leq e^{\frac{1}{2}\left(f, \Gamma_{j} f\right)-\alpha q\left(\delta_{x_{0}}, \Gamma_{j} f\right)} \mathbb{E}_{j}\left[\left\|\widehat{K}_{j}(q, \varphi+i \delta \psi, X)\right\|_{h, T_{j+1}\left(\varphi^{\prime}, X\right)}\right] .
\end{aligned}
$$

With [14], choose $f_{x}=\alpha \operatorname{sgn}(q) \delta_{x, x_{0}}$ (see the Remark below) and obtain

$$
\begin{aligned}
& \left\|\mathbb{E}_{j}\left[\widehat{K}_{j}(q, \varphi, X)\right]\right\|_{h, T_{j+1}\left(\varphi^{\prime}, X\right)} \\
& \quad \leq e^{-\left(|q|-\frac{1}{2}\right) \alpha^{2} \Gamma_{j}(0)} \mathbb{E}_{j}\left[\left\|\widehat{K}_{j}(q, \varphi+i \delta \psi, X)\right\|_{h, T_{j+1}\left(\varphi^{\prime}, X\right)}\right] .
\end{aligned}
$$

This inequality is an important result of [14]. Now consider the expectation on the r.h.s. of (E.9): for $\rho:=5 L^{-1}, \eta:=\zeta+i \delta \psi, \Delta:=\|\delta \psi\|_{\mathcal{C}_{j}^{2}(X)}$, since $\left\|e^{i q \varphi_{x_{0}}}\right\|_{h, T_{j+1}\left(\varphi^{\prime}, X\right)}$ is less than $e^{h|q| \alpha}$, by (4.3), (E.2), (E.3), (B.3), and for $L$ so large that $\rho h+\Delta \leq h$,

$$
\begin{aligned}
\left\|\widehat{K}_{j}(q, \varphi+i \delta \psi, X)\right\|_{h, T_{j+1}\left(\varphi^{\prime}, X\right)} \leq e^{h|q| \alpha}\left\|\widehat{K}_{j}\left(q, \delta \varphi^{\prime}+\eta, X\right)\right\|_{h, T_{j+1}\left(\varphi^{\prime}, X\right)} \\
\leq\left. e^{h|q| \alpha}\left\|\widehat{K}_{j}(q, \xi+\eta, X)\right\|_{\rho h, T_{j}(\xi, X)}\right|_{\xi:=\delta \varphi^{\prime}} \\
\leq\left. e^{h|q| \alpha}\left\|\widehat{K}_{j}(q, \xi+\zeta, X)\right\|_{\rho h+\Delta, T_{j}(\xi, X)}\right|_{\xi:=\delta \varphi^{\prime}} \\
\leq e^{h|q| \alpha}\left\|K_{j}(X)\right\|_{h, T_{j}(X)} G_{j}(\varphi, X) .
\end{aligned}
$$

(The final inequality is a consequence of the fact that $G_{j}(\varphi, X)$ depends on the derivatives of $\varphi$, and then $G_{j}(\xi+\zeta, X)=G_{j}(\varphi, X)$.) Finally, plugging (E.10) into (E.9), for any $\vartheta>0$ and $L$ large enough

$$
\begin{aligned}
& \left\|\mathbb{E}_{j}\left[\widehat{K}_{j}(q, \varphi, X)\right]\right\|_{h, T_{j+1}\left(\varphi^{\prime}, X\right)} \\
& \quad \leq e^{c(\alpha)|q|} L^{-(2|q|-1) \frac{\alpha^{2}}{4 \pi}}\left\|K_{j}\right\|_{h, T_{j}}\left(\frac{A}{2}\right)^{-|X|_{j}} G_{j+1}\left(\varphi^{\prime}, \bar{X}\right) .
\end{aligned}
$$

This inequality proves Theorem 5.6 .

Remark. In order to minimize the prefactor in the r.h.s. of (E.8), one should rather choose $f_{x}=\alpha q \delta_{x, x_{0}}$; then, the prefactor in $(\underline{E .9})$ would be $e^{-\frac{q^{2}}{2} \alpha^{2} \Gamma_{j}(0)}$, as in heuristic arguments. Though this choice conflicts with the condition $\rho h+\Delta \leq h$, for the corresponding $\Delta$ grows with $q$, whereas the radius of analyticity, $h$, is assumed independent of $q$. 


\section{E.2 Proof of Theorem 5.5}

With Taylor remainder in $\delta \varphi^{\prime}$, by (E.2), (E.4),

$$
\begin{aligned}
& \left\|\operatorname{Rem}_{2} \widehat{K}_{j}\left(0, \delta \varphi^{\prime}+\zeta, X\right)\right\|_{h, T_{j+1}\left(\varphi^{\prime}, X\right)} \leq\left.\left\|\operatorname{Rem}_{2} \widehat{K}_{j}(0, \xi+\zeta, X)\right\|_{\rho h, T_{j}(\xi, X)}\right|_{\xi=\delta \varphi^{\prime}} \\
& \leq\left.\left(1+(\rho h)^{-1}\|\xi\|_{\mathcal{C}_{j}^{2}(X)}\right)^{3} \sup _{t \in[0,1]}\left\|\widehat{K}_{j}(0, t \xi+\zeta, X)\right\|_{\rho h, T_{j}^{23}(t \xi, X)}\right|_{\xi=\delta \varphi^{\prime}} \\
& \leq\left. C\left(1+L\|\xi\|_{\mathcal{C}_{j}^{2}(X)}\right)^{3} \rho^{3} \sup _{t \in[0,1]}\left\|\widehat{K}_{j}(0 ; t \xi+\zeta, X)\right\|_{h, T_{j}(t \xi, X)}\right|_{\xi=\delta \varphi^{\prime}} \\
& \leq C^{\prime}\left(1+L\|\xi\|_{\mathcal{C}_{j}^{2}(X)}\right)^{3} L^{-3}\left\|K_{j}(X)\right\|_{h, T_{j}(X)} \sup _{t \in[0,1]} G_{j}\left(t \varphi^{\prime}+\zeta, X\right)
\end{aligned}
$$

(We used that, as $\rho=5 L^{-1}<1$, then $\|\cdot\|_{\rho h, T_{j}^{\geq 3}} \leq \rho^{3}\|\cdot\|_{h, T_{j}}$.) As $X \in \mathcal{S}_{j}$,

$$
L\left\|\delta \varphi^{\prime}\right\|_{\mathcal{C}_{j}^{2}(X)} \leq C \max _{p=1,2}\left\|\nabla_{j+1}^{p} \varphi^{\prime}\right\|_{L^{\infty}\left(X^{*}\right)} .
$$

Therefore, by (4.3), (5.17)

$$
\begin{aligned}
& \| \operatorname{Rem}_{2} \mathbb{E}_{j} {\left[\widehat{K}_{j}(0, \varphi, X)\right] \|_{h, T_{j+1}\left(\varphi^{\prime}, X\right)} } \\
& \leq C \frac{L^{-3}}{\kappa_{L}^{3 / 2}}\left\|K_{j}(X)\right\|_{h, T_{j}(X)}\left(\frac{A}{2}\right)^{-|X|_{j}} G_{j+1}\left(\varphi^{\prime}, \bar{X}\right),
\end{aligned}
$$

which proves Theorem 5.5.

\section{E.3 Proof of Theorem 5.7}

Set $\eta:=\zeta+i \delta \psi$. By (E.2), (E.4), (E.3), and since, by definition of $h, \frac{h}{2}+\Delta \leq h$,

$$
\begin{aligned}
\left\|\operatorname{Rem}_{0} \widehat{K}_{j}\left(q, \delta \varphi^{\prime}+\eta, X\right)\right\|_{h, T_{j+1}\left(\varphi^{\prime}, X\right)} \leq\left.\left\|\operatorname{Rem}_{0} \widehat{K}_{j}(q, \xi+\eta, X)\right\|_{\rho h, T_{j}(\xi, X)}\right|_{\xi=\delta \varphi^{\prime}} \\
\leq\left. C\left(1+L\|\xi\|_{\mathcal{C}_{j}^{2}(X)}\right) \sup _{0 \leq t \leq 1}\left\|\widehat{K}_{j}(q, t \xi+\eta, X)\right\|_{\rho h, T_{j}^{\geq 1}(\xi, X)}\right|_{\xi=\delta \varphi^{\prime}} \\
\leq C^{\prime}\left(1+L\|\xi\|_{\mathcal{C}_{j}^{2}(X)}\right) L^{-1} \sup _{0 \leq t \leq 1}\left\|\widehat{K}_{j}(q, t \xi+\eta, X)\right\|_{\frac{h}{2}, T_{j}(\xi, X)} \\
\leq C^{\prime}\left(1+L\|\xi\|_{\mathcal{C}_{j}^{2}(X)}\right) L^{-1} \sup _{0 \leq t \leq 1}\left\|\widehat{K}_{j}(q, t \xi+\zeta, X)\right\|_{h, T_{j}(\xi, X)} \\
\leq C^{\prime}\left(1+L\|\xi\|_{\mathcal{C}_{j}^{2}(X)}\right) L^{-1}\left\|K_{j}(X)\right\|_{h, T_{j}} \sup _{0 \leq t \leq 1} G_{j}\left(t \varphi^{\prime}+\zeta, X\right) .
\end{aligned}
$$

In analogy with the derivation of (E.9),

$$
\begin{aligned}
& \| \mathbb{E}_{j}[\left.\operatorname{Rem}_{0} \widehat{K}_{j}(q, \varphi, X)\right] \|_{h, T_{j+1}\left(\varphi^{\prime}, X\right)} \\
& \quad \leq e^{-\left(|q|-\frac{1}{2}\right) \alpha^{2} \Gamma_{j}(0)} \mathbb{E}_{j}\left[\left\|\operatorname{Rem}_{0} \widehat{K}_{j}(q, \varphi+i \delta \psi, X)\right\|_{h, T_{j+1}\left(\varphi^{\prime}, X\right)}\right] .
\end{aligned}
$$


Then, continuing the chain of inequalities by (E.13) and (E.15), (5.17),

$$
\begin{aligned}
& \left\|\mathbb{E}_{j}\left[\operatorname{Rem}_{0} \widehat{K}_{j}(q, \varphi, X)\right]\right\|_{h, T_{j+1}\left(\varphi^{\prime}, X\right)} \\
& \leq e^{-\left(|q|-\frac{1}{2}\right) \alpha^{2} \Gamma_{j}(0)} \mathbb{E}_{j}\left[\left\|\operatorname{Rem}_{0} \widehat{K}_{j}(q, \varphi+i \delta \psi, X)\right\|_{\left.h, T_{j+1}\left(\varphi^{\prime}, X\right)\right]}\right] \\
& \leq e^{-\left(|q|-\frac{1}{2}\right) \alpha^{2} \Gamma_{j}(0)} e^{h|q| \alpha} \mathbb{E}_{j}\left[\left\|\operatorname{Rem}_{0} \widehat{K}_{j}\left(q, \delta \varphi^{\prime}+\eta, X\right)\right\|_{\left.h, T_{j+1}\left(\varphi^{\prime}, X\right)\right]}\right. \\
& \leq C e^{-\left(|q|-\frac{1}{2}\right) \alpha^{2} \Gamma_{j}(0)} e^{h|q| \alpha} \frac{L^{-1}}{\kappa_{L}^{3 / 2}}\left\|K_{j}\right\|_{h, T_{j}}\left(\frac{A}{2}\right)^{-|X|_{j}} G_{j+1}\left(\varphi^{\prime}, \bar{X}\right),
\end{aligned}
$$

which proves Theorem 5.7 .

Acknowledgements It is a pleasure to thank David Brydges for patient guidance during the study of [5], clarifying discussions on [14] and [10], many important suggestions - including a crucial one labeled as reference [7]; and, most of all, for constant support during the (long) writing process. This work is supported by the Giorgio and Elena Petronio Fellowship Fund.

\section{References}

[1] Amit D.J., Goldschmidt Y., Grinstein G.: Renormalisation group analysis of the phase transition in the 2D Coulomb gas, Sine-Gordon theory and XY-model. J.Phys.A 13 585-620 (1980).

[2] Benfatto G., Gallavotti G., Nicolò F.: The dipole phase in the twodimensional hierarchical Coulomb gas: analyticity and correlations decay. Comm.Math.Phys. 106 277-288 (1986).

[3] Benfatto G., Renn J.: Nontrivial fixed points and screening in the hierarchical two-dimensional Coulomb gas. J.Stat. Phys. 67 957-980 (1992).

[4] Berezinskii V.L.: Destruction of long-range order in one-dimensional and twodimensional systems possessing a continuous symmetry group II. Quantum systems Sov.Phys. JETP, 34, 610, (1971)

[5] Brydges D.C.: Lectures on the Renormalisation Group Park City Lectures. (2007)

[6] Brydges D.C: A rigorous approach to Debye screening in dilute classical coulomb systems Comm.Math.Phys.58 313-350 (1978).

[7] Brydges D.C: private communication.

[8] Brydges D.C., Federbush P.: Debye screening Comm.Math.Phys. 129 351-392 (1990) 
[9] Brydges, D.C., Guadagni G., Mitter P.K.: Finite Range Decomposition of Gaussian Processes. J.Stat.Phys. 115 415-449 (2004).

[10] Brydges, D.C., Slade G.: Weakly self-avoiding walk in dimensions four and higher: a renormalisation group analysis. In preparation.

[11] Brydges D.C., Yau H.T.: Grad $\varphi$ perturbations of massless Gaussian fields. Comm.Math.Phys. 73 197-246 (1980)

[12] Dimock J. The Kosterlitz-Thouless phase in a hierarchical model. J.Phys.A 23 1207 (1990).

[13] Dimock J. Infinite Volume Limit for the Dipole Gas. J.Stat.Phys. 135393427 (2009).

[14] Dimock J., Hurd T.R.: Sine-Gordon Revisited. Ann.H.Poincare 541 499-541 (2000).

[15] Deutsch C., Lavaud M.: Equilibrium properties of a two-dimensional Coulomb gas. Phys.Rev.A 9 2598-2616 (1974).

[16] Guidi L.F., Marchetti D.H.U.: Renormalization Group Flow of the TwoDimensional Hierarchical Coulomb Gas Comm.Math.Phys. 219 671-702 (2001).

[17] Fröhlich J., Park Y.M.: Correlation inequalities and the thermodynamic limit for classical and quantum continuous systems Comm.Math.Phys. 59 235-266 (1978).

[18] Fröhlich J., Spencer T.: On the statistical mechanics of classical Coulomb and dipole gases. J.Stat.Phys. 24 617-701 (1981).

[19] Fröhlich J., Spencer T.: The Kosterlitz-Thouless transition in two-dimensional Abelian spin systems and the Coulomb gas. Comm.Math.Phys. 81 527-602 (1981)

[20] Gallavotti G., Nicolò F.: The screening phase transitions in the twodimensional Coulomb gas J.Stat.Phys. 39 133-156 (1985)

[21] José V.J., Kadanoff L.P., Kirkpatrick S. and Nelson D.R.: Renormalization, vortices, and symmetry-breaking perturbations in the two-dimensional planar model. Phys.Rev.B 16 1217-1241 (1977).

[22] Kadanoff L.P.: Lattice coulomb gas representations of two-dimensional problems. J.Phys.A 11 (1978).

[23] Kadanoff L.P.: Statistical Physics. Statics, Dynamics and Renormalization. World Scientific - Singapore (1999). 
[24] Kappeler T., Pinn K. and Wieczerkowski C.: Renormalization group flow of a hierarchical Sine-Gordon model by partial differential equations. Comm.Math.Phys. 136 357-368 (1991).

[25] Kosterlitz J. M.: The critical properties of the two-dimensional xy model J.Phys.C 71046 (1974)

[26] Kosterlitz J. M., Thouless D. J.: Ordering, metastability and phase transitions in two-dimensional systems J.Phys.C 61181 (1973)

[27] Marchetti D.H.U.: Comments on "power-law falloff in the Kosterlitz-Thouless phase of a two-dimensional lattice Coulomb gas" J.Stat.Phys. 61 909-911 (1990).

[28] Marchetti D.H.U., Klein A.: Power-law falloff in two-dimensional Coulomb gases at inverse temperature $>8 \pi$. J.Stat.Phys. 64 135-162 (1991)

[29] Marchetti D.H.U., Klein A., Perez J.F.: Power-law falloff in the KosterlitzThouless phase of a two-dimensional lattice Coulomb gas J.Stat.Phys. 60 137166 (1990)

[30] Marchetti D.H.U., Perez J.F.: The Kosterlitz-Thouless phase transition in twodimensional hierarchical Coulomb gases J.Stat.Phys. 55 141-156 (1989).

[31] Mitter P.K., Scoppola B.: Renormalization group approach to interacting polymerised manifolds. Comm.Math.Phys. 209 207-261 (2000).

[32] Nienhuis B.: Coulomb gas formulation of two-dimensional phase transitions in Phase transitions- Vol 11 Ed: Domb C, Lebowitz J L. (1987)

[33] Nicolò F., Perfetti P.: The sine-Gordon field theory model at $\alpha^{2}=8 \pi$, the nonsuperrenormalizable theory. Comm.Math.Phys. 123 425-452 (1989).

[34] Tellez, G: Equation of State in the Fugacity Format for the Two-Dimensional Coulomb Gas J.Stat.Phys. 126 281-298 (2007).

[35] Yang W.S.: Debye screening for two-dimensional Coulomb systems at high temperatures J.Stat.Phys. 49 1-32 (1987) 